CRYSTALLOGRAPHIC COMMUNICATIONS

ISSN 2056-9890

Received 31 May 2017

Accepted 7 June 2017

Edited by J. P. Jasinsk, Keene State College, USA

Keywords: crystal structure; naltrexone; chloride; solvate; ethanol; propan-2-ol; 2methylpropan-2-ol.

CCDC references: $1554631 ; 1554630$; 1554629

Supporting information: this article has supporting information at journals.iucr.org/e

\section{Crystal structure of naltrexone chloride solvates with ethanol, propan-2-ol, and 2-methylpropan-2-ol}

\author{
Aveary R. Menze, Jefferson P. Sinnott and Alexander Y. Nazarenko* \\ Chemistry Department, SUNY Buffalo State, 1300 Elmwood Ave, Buffalo, NY 14222, USA. *Correspondence e-mail: \\ nazareay@buffalostate.edu
}

Naltrexone [systematic name: 17-(cyclopropylmethyl)-3,14-dihydroxy-4,5 $\alpha$ epoxymorphinan-6-one] is an opioid receptor competitive antagonist that has been widely used to prevent relapse in opioid- and alcohol-dependent subjects. Its chloride salt forms non-isomorphic solvates with ethanol $\left(\mathrm{C}_{20} \mathrm{H}_{24} \mathrm{NO}_{4}{ }^{+} \cdot \mathrm{Cl}^{-}\right.$.$\left.\cdot \mathrm{C}_{2} \mathrm{H}_{5} \mathrm{OH}\right)$ (I), propan-2-ol $\left(\mathrm{C}_{20} \mathrm{H}_{24} \mathrm{NO}_{4}{ }^{+} \cdot \mathrm{Cl}^{-} \cdot \mathrm{C}_{3} \mathrm{H}_{7} \mathrm{OH}\right)$ (II), and 2-methylpropan-2-ol $\left(\mathrm{C}_{20} \mathrm{H}_{24} \mathrm{NO}_{4}{ }^{+} \cdot \mathrm{Cl}^{-} \cdot \mathrm{C}_{4} \mathrm{H}_{9} \mathrm{OH}\right)$ (III). The naltrexone cation can be described as a T-shape made out of two ring systems, a tetrahydro- $2 \mathrm{H}$ naphtho[1,8-bc]furan system and a decahydroisoquinolinium subunit, that are nearly perpendicular to one another. The flexible cyclopropylmethyl group can adopt various different conformations in response to its surroundings: an increase of available space around cyclopropylmethyl group may allow it to adopt a more favorable conformation. In all these structures, the alcohol molecules occupy infinite solvent-filled channels. All three compounds described are attractive crystalline forms for unambiguous identification of naltrexone chloride after isolation from a pharmaceutical form. Compound (III) was refined as a two-component twin.

\section{Chemical context}

Alcohol and opiate dependence are potentially life-threatening disorders associated with adverse physical and societal effects including poor social functioning, familial problems, and crime (Compton \& Volkow, 2006). One strategy suggested to address these issues is the inclusion of receptor antagonists that reduce, and can even reverse, the euphoric effects of the drug sought by abusers. Naltrexone [systematic name: 17(cyclopropylmethyl)-3,14-dihydroxy-4,5 $\alpha$-epoxy-morphinan-6one] is an opioid receptor competitive antagonist that has been widely used to prevent relapse in heroin and other opioiddependent subjects, and has been found to reduce cravings in alcohol-dependent subjects (Roozen et al., 2006). Its structurerelated analogue oxymorphone is a potent $\mu$-agonist, which differs from naltrexone only in having an $N$-methyl group in place of an $N$-cyclopropylmethyl group (Amato et al., 1990). Elucidation of the conformational profile of naltrexone is of fundamental importance in order to determine molecular requirements for the specific binding affinities of this drug, particularly through the possible position of groups responsible for pharmacological action.

The most common pharmaceutical form of this compound is naltrexone hydrochloride tablets. The introduction of new crystalline forms of an active pharmaceutical compound provides an opportunity to improve the performance characteristics of a pharmaceutical product. There is a need for new crystalline forms of naltrexone hydrochloride (Nichols $e t$ 
al., 2013) as well for new analytical methods of its unambiguous identification. This communication is a continuation of our work on analytical crystallography of opiate compounds (Gauchat \& Nazarenko, 2017).

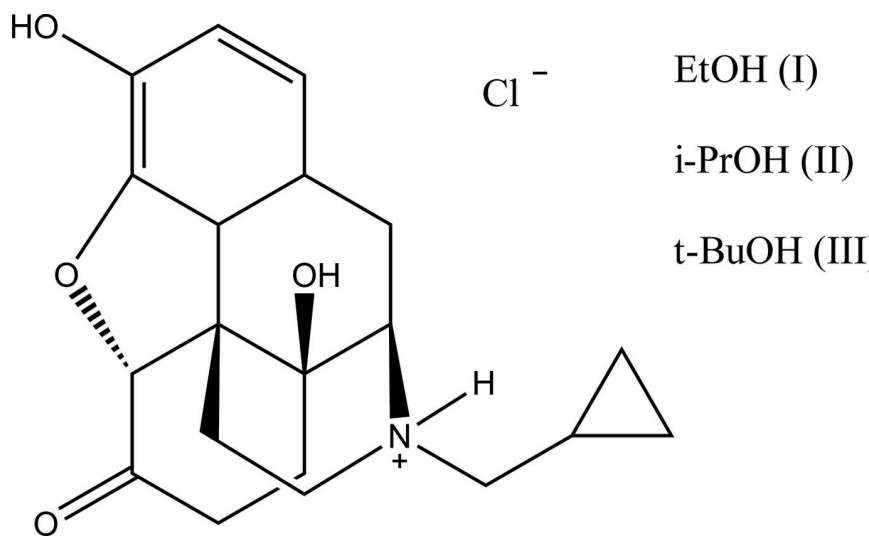

\section{Structural commentary}

In all cases, interaction with the alcohol molecules does not affect the geometry of the methorphan ring system (Fig. 1), leaving the shape of the organic molecule intact. The bond

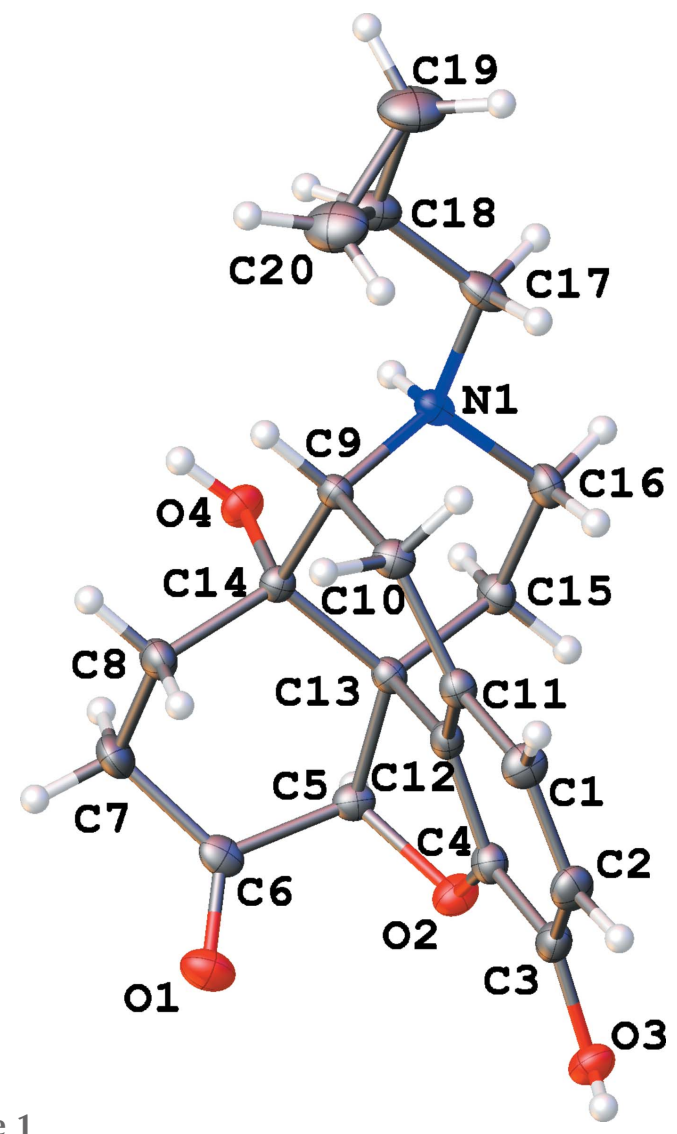

Figure 1

The numbering scheme of the naltrexone cation in the ethanol solvate structure (I), with $50 \%$ probability ellipsoids. All other naltrexone cations have the same numbering scheme (100 added to each atom number in a second naltrexone cation in structure III).
Table 1

Ring puckering analysis $\left(\AA,^{\circ}\right)$ of five- and six-membered rings.

Ring $A$ dihydrofuran (atoms $\mathrm{O} 2 / \mathrm{C} 4 / \mathrm{C} 12 / \mathrm{C} 13 / \mathrm{C} 5$ ), ring $B$ piperidine (atoms N1/C9/C14/C13/C15/C16), ring $C$ cyclohexanone (atoms C5/C6/C7/C8/C13/ C14) and ring $D$ cyclohexadiene (atoms C9/C10/C11/C12/C13/C14).

\begin{tabular}{llllll}
\hline Ring & parameter & (I) & (II) & (III) cation 1 & (III) cation 2 \\
\hline \multirow{2}{*}{$A$} & $Q$ & $0.341(2)$ & $0.340(3)$ & $0.313(3)$ & $0.341(3)$ \\
& $\varphi$ & $314.5(4)$ & $314.3(5)$ & $310.6(5)$ & $314.4(5)$ \\
$B$ & $Q$ & $0.637(2)$ & $0.624(3)$ & $0.637(3)$ & $0.636(3)$ \\
& $\theta$ & $11.28(18)$ & $10.9(3)$ & $9.3(3)$ & $9.6(3)$ \\
& $\varphi$ & $101.0(9)$ & $110.8(14)$ & $102.1(15)$ & $97.6(15)$ \\
$C$ & $Q$ & $0.546(3)$ & $0.509(3)$ & $0.509(3)$ & $0.516(3)$ \\
& $\theta$ & $157.3(2)$ & $157.7(3)$ & $155.8(3)$ & $158.5(3)$ \\
& $\varphi$ & $322.5(7)$ & $343.9(10)$ & $349.1(9)$ & $340.4(10)$ \\
$D$ & $Q$ & $0.495(2)$ & $0.502(3)$ & $0.499(3)$ & $0.508(3)$ \\
& $\theta$ & $131.6(2)$ & $134.1(3)$ & $134.2(3)$ & $132.1(3)$ \\
& $\varphi$ & $121.2(3)$ & $122.7(5)$ & $123.7(5)$ & $122.4(4)$ \\
\hline
\end{tabular}

lengths and angles in the alcohol solvates are not far from expected values and are generally close to those reported for the hydrate structure (Ledain et al., 1992).

There are four six-membered rings and a five-membered ring in a naltrexone molecule. The aromatic ring is close to planar, with deviations less than $0.03 \AA$ in all cases. The cyclohexadiene ring can be described as a half-chair shifted towards an envelope conformation: atoms $\mathrm{C} 10, \mathrm{C} 11, \mathrm{C} 12$ and $\mathrm{C} 13$ are adjacent to the aromatic ring and therefore almost planar while $\mathrm{C} 9$ and $\mathrm{C} 14$ deviate from this plane in opposite directions (see Table 1 for puckering parameters). A similar observation is true for the dihydrofurane five-membered ring, which is almost intermediate between an envelope and a halfchair with $\mathrm{C} 5$ and $\mathrm{C} 13$ deviating from the mean plane in opposite directions.

The cyclohexanone and piperidine rings both have chair conformations, with cyclohexanone visibly shifted towards half-chair. These two rings are nearly coplanar. As a result, the naltrexone cation can be described as having two ring systems: a phenyl ring with adjacent epoxy and cyclohexadiene rings

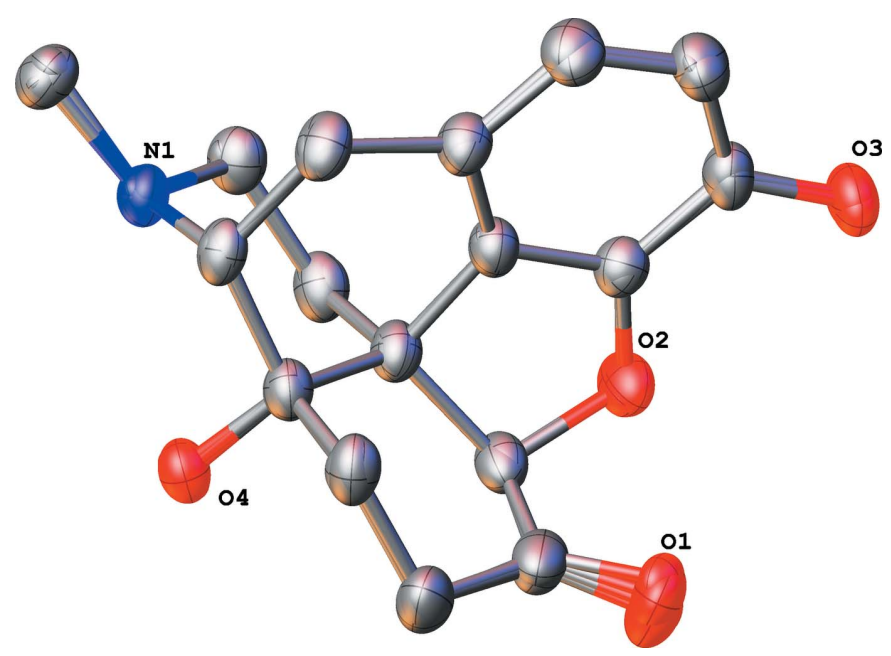

Figure 2

Overlay of all four naltrexone cations studied in this work with the cyclopropyl group omitted. 
(tetrahydro-2H-naphtho[1,8-bc]furan system, atoms $\mathrm{O} 2 / \mathrm{C} 1-$ $\mathrm{C} 4 / \mathrm{C} 9-\mathrm{C} 13)$ and cyclohexanone plus piperidine rings (decahydroisoquinolinium moiety, atoms N1/C5-C9/C13-C16). They are nearly perpendicular to each other, thus forming the well-established T-shape common to morphine, naloxene, and numerous similar molecules (Darling et al., 1982; Klein et al., 1987; Gelbrich et al., 2012). The angle between two mean planes is $83.9(1)^{\circ}$ for $\mathrm{EtOH}(\mathrm{I}), 83.4(1)^{\circ}$ for $i-\mathrm{PrOH}$ (II) and 82.5 (1) and $84.3(1)^{\circ}$ for the two cations in $t-\mathrm{BuOH}$ (III) solvate.

What is responsible for switching from a potent opiate agonist (morphine and oxymorphone) to a potent competitive antagonist (naloxene and naltrexone)? It seems certain that changes in a relatively rigid oxymorphone cation are not liable. Overlay calculations show that all three naltrexone solvates fit the same shape (Fig. 2), with r.m.s. deviations being $0.09(\mathrm{EtOH} / i-\mathrm{PrOH}), 0.06$ and $0.11 \AA(\mathrm{EtOH} / t-\mathrm{BuOH})$. The same overlay with an oxymorphone cation (refcode BIZGAS) shows r.m.s. deviations of 0.10 to $0.13 \AA$ and $0.13 \AA$ for naloxene (refcode NALOXC02). It should be taken into account that, in these cases, the temperature of the experiment was different, which obviously increases the discrepancy. Even when we compare morphine (refcode EFASAH; Gelbrich et al., 2012) and oxymorphone and morphine and naltrexone, the fit is almost identical: r.m.s. deviations of 0.36 and $0.35 \AA$, respectively, with larger discrepancies coming from obvious

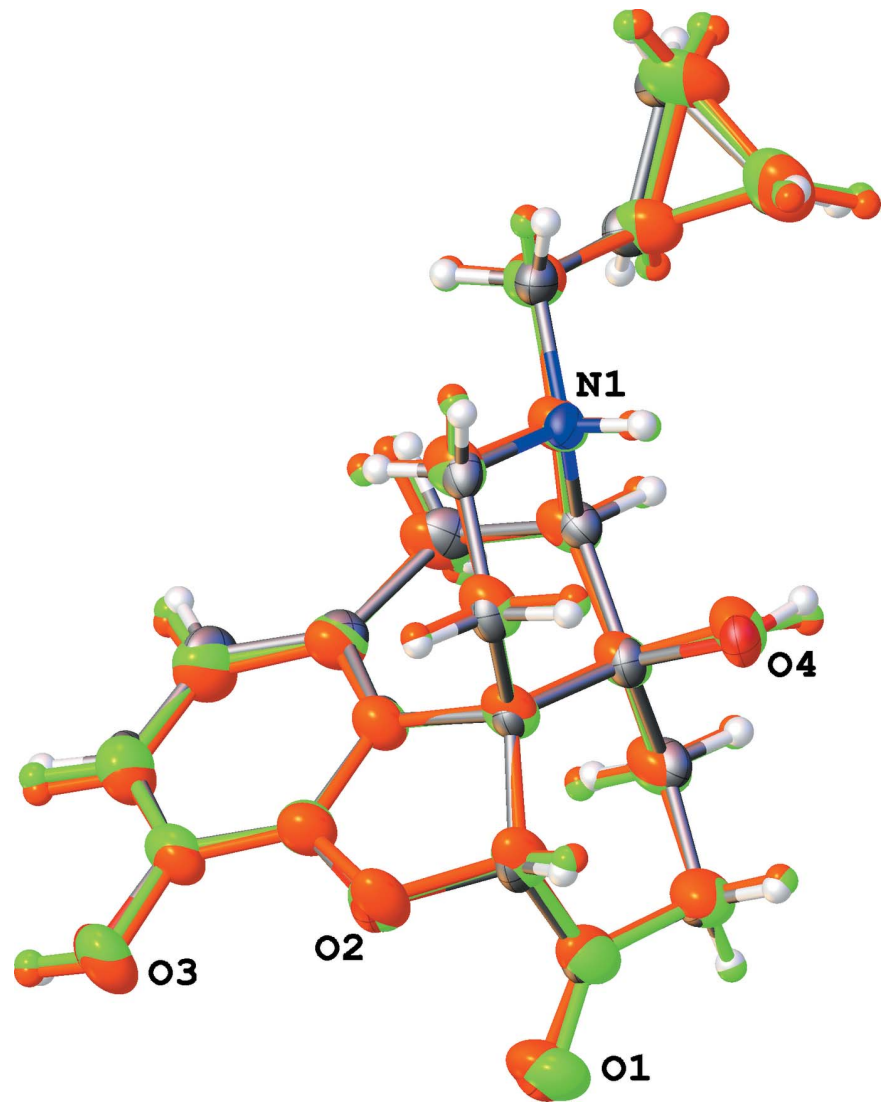

Figure 3

Overlay of both naltrexone cations of the tert-butanol solvate (III) (red and green) and of the propan-2-ol solvate (II) (usual color scheme). The orientation of the cyclopropyl group is similar in all three cases.

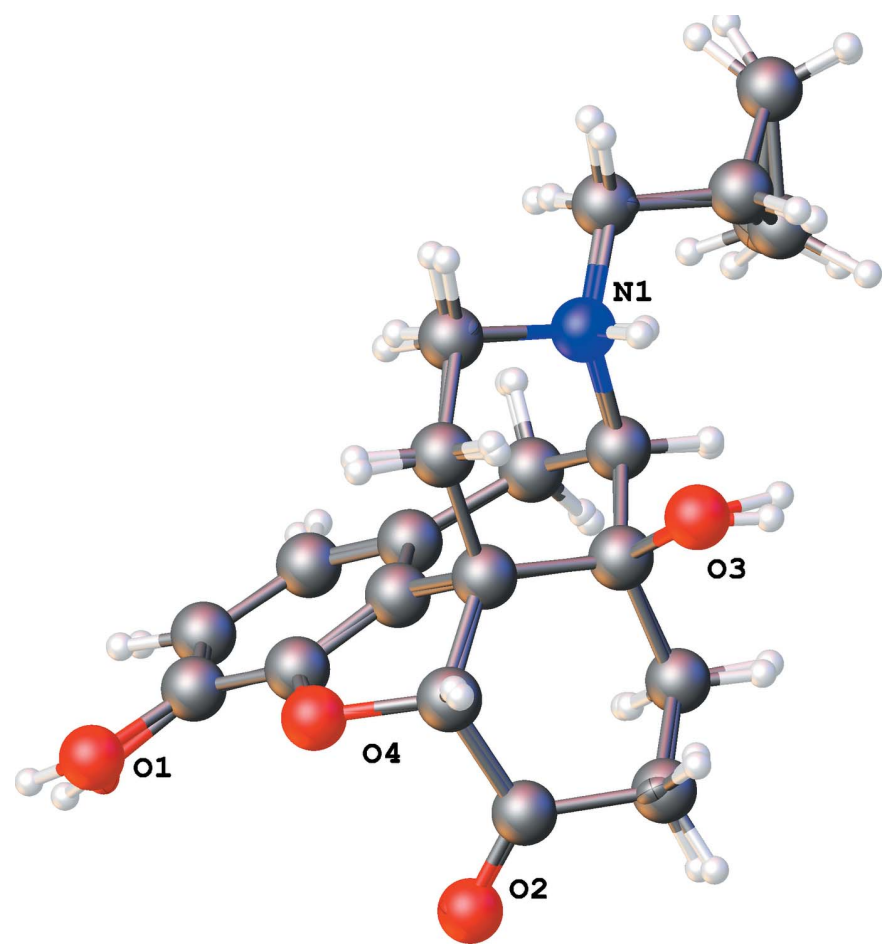

Figure 4

Overlay of the naltrexone cations of the ethanol solvate (I) and the tetrahydrate (refcode PABCEA). The orientation of the cyclopropyl group is similar in both cases.

structural differences between the phenol group of morphine and a cyclohexanone fragment in oxymorphone and naltrexone. The only flexible locations in the oxymorphone cation are oxygen $\mathrm{O} 1$ of the carbonyl group and the orientation of two hydroxyl groups (oxygen atoms $\mathrm{O} 3$ and $\mathrm{O} 4$ ), which all potentially form strong hydrogen bonds.

Therefore, the simplest explanation of antagonist activity is the presence of a small 'flat' fragment attached to an $N$-methyl

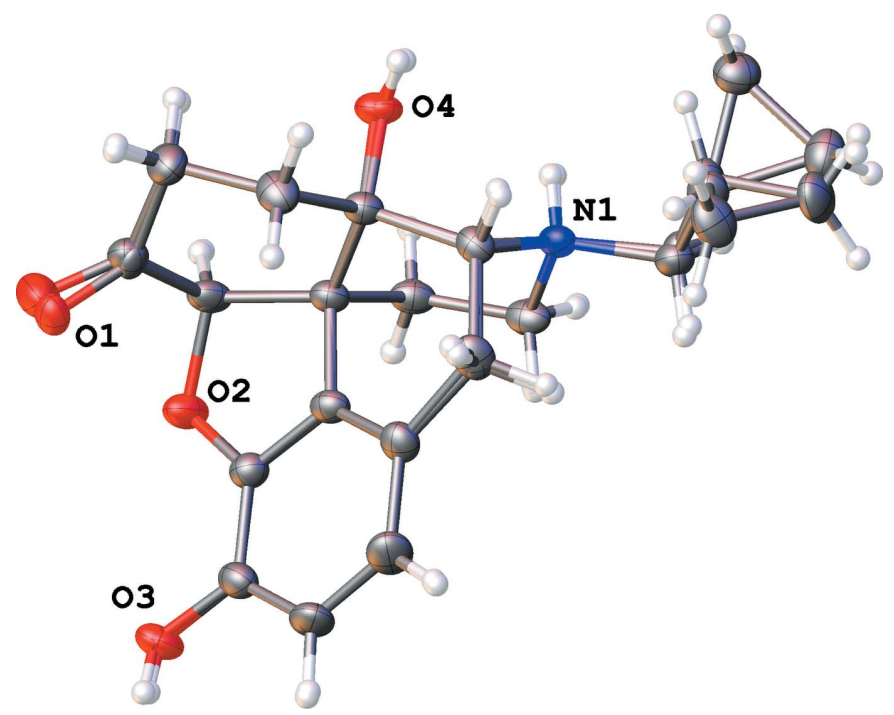

Figure 5

Overlay of the naltrexone cations of the ethanol solvate (I) and propanol solvate (II). The orientation of the cyclopropyl group is visibly different. 
Table 2

Hydrogen-bond geometry $\left(\AA{ }^{\circ}\right)$ for $(\mathrm{I})$.

\begin{tabular}{lllll}
\hline$D-\mathrm{H} \cdots A$ & $D-\mathrm{H}$ & $\mathrm{H} \cdots A$ & $D \cdots A$ & $D-\mathrm{H} \cdots A$ \\
\hline $\mathrm{N} 1-\mathrm{H} 1 \cdots \mathrm{O} 1^{\mathrm{i}}$ & $0.85(3)$ & $2.23(2)$ & $2.870(2)$ & $133(2)$ \\
$\mathrm{O} 3-\mathrm{H} 3 \cdots \mathrm{Cl} 01$ & $0.80(2)$ & $2.23(2)$ & $3.0297(17)$ & $171(2)$ \\
$\mathrm{O} 4-\mathrm{H} 4 \cdots \mathrm{Cl} 1{ }^{\mathrm{ii}}$ & $0.81(3)$ & $2.36(3)$ & $3.1279(17)$ & $159(3)$ \\
$\mathrm{O} 5-\mathrm{H} 5 A \cdots \mathrm{Cl} 01$ & 0.84 & 2.33 & $3.160(2)$ & 169 \\
\hline
\end{tabular}

Symmetry codes: (i) $x, y-1, z$; (ii) $x+1, y-1, z$.

group: cyclopropyl in naltrexone or vinyl in naloxene. The link between this small rigid fragment and the oxymorphone cation is flexible: as a result, we see different orientations of the cyclopropane ring in various solvates of naltrexone. These orientations can be systemized in two groups. First, an overlay of the hydrate (refcode PABCEA) and the ethanol solvate (this work) shows very similar conformations for these two structures (Fig. 3). The orientation of the cyclopropyl group in the iso-propanol and tert-butanol solvates is also almost the same (Fig. 4). However, these two groups significantly differ from each other (Fig. 5). The angle between the cyclopropyl group plane and the mean plane of the cyclohexanone and piperidine rings can serve as a quantitative measure of the methylcyclopropyl fragment orientation. This angle is $36.1^{\circ}$ (formate, $\left.\mathrm{H}_{2} \mathrm{O}\right), 38.6^{\circ}\left(\mathrm{H}_{2} \mathrm{O}\right), 48.6^{\circ}(\mathrm{EtOH}), 71.7^{\circ}(i-\mathrm{PrOH})$, $83.5^{\circ}$ and $84.6^{\circ} \AA(t$-BuOH $)$; the first two values were calculated from Scheins et al. (2007) and Ledain et al. (1992). Thus, the conformation of the methylcyclopropyl fragment is very sensitive to its environment.

\section{Supramolecular features}

The way in which a solvate molecule interacts with a naltrexone cation is different in all cases studied. Obviously, the strongest possible interaction is a hydrogen bond associated with the hydroxyl group of the alcohol molecule. However, naltrexone hydrochloride is an ionic compound and

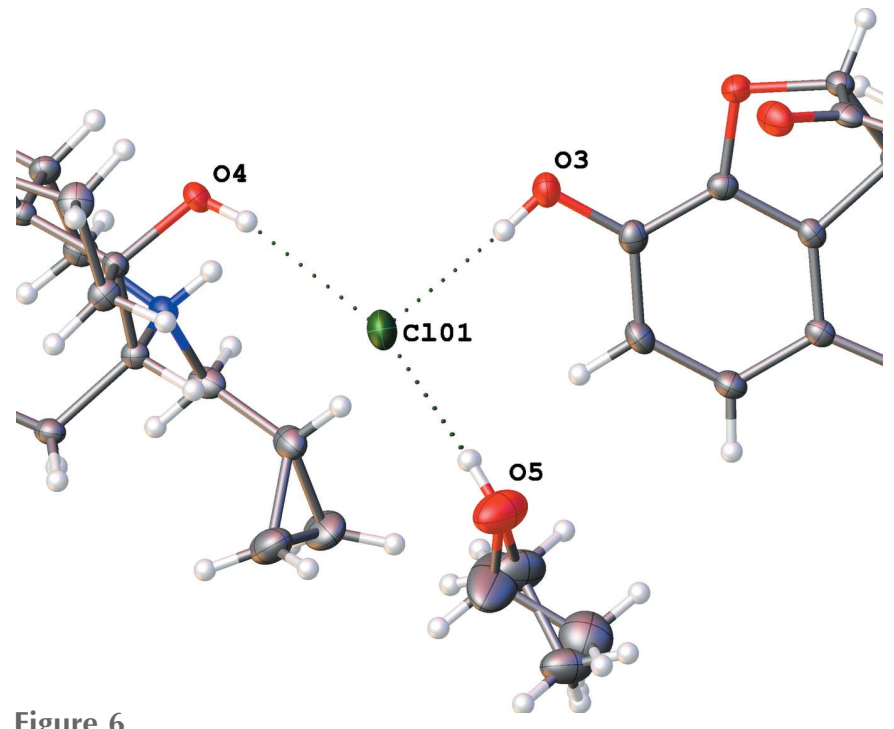

Hydrogen bonds around the chloride ion in the ethanol solvate (I).

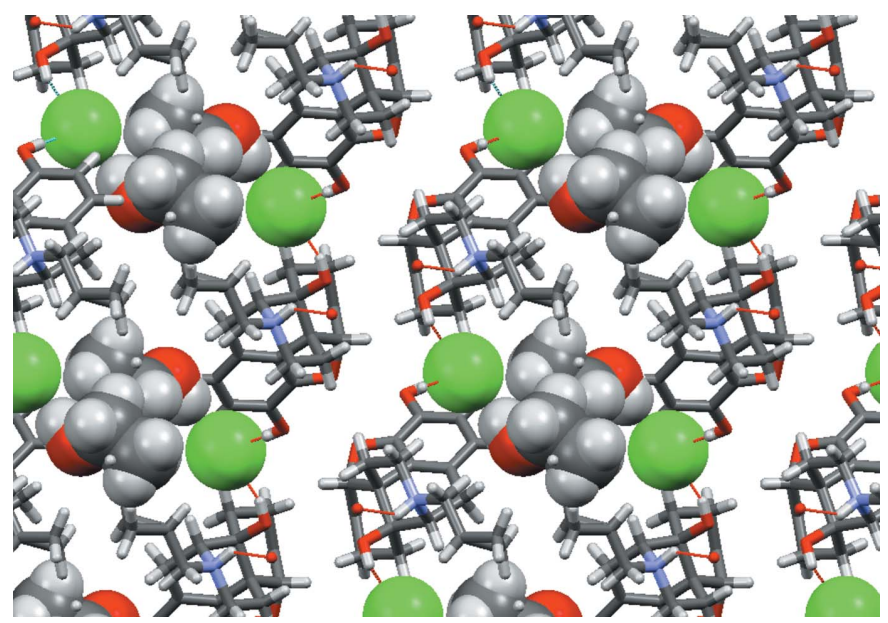

Figure 7

Packing diagram of the ion associates in the ethanol solvate (I), viewed along [010]. There is a visible gap between the bilayers. Chloride ions (green) and ethanol molecules are highlighted.

electrostatic interaction between a positively charged bulk cation and a chloride ion plays an essential role in crystal formation. Electrostatic potential data (Scheins et al., 2007) show more or less uniform positive charge for most of the cation surface, with the obvious exception of the negatively charged oxygen atoms.

In the ethanol solvate (I), the ethanol molecule is disordered; however, both orientations show strong hydrogen bonds with the chloride anion and no direct interaction with the naltrexone cation. The chloride ion is surrounded by hydroxyl groups belonging to two different cations (Fig. 6, Table 1). Interestingly, there is no hydrogen bond between the chloride ion and the formally positively charged protonated ammonium nitrogen atom N1. Instead, there is a strong hydrogen bond between $\mathrm{N} 1$ and oxygen atom $\mathrm{O} 1$ of the carbonyl group belonging to another cation (Table 2). As a result, the naltrexone cations form infinite chains along the [010] direction. These chains are bound together via hydrogen bonds involving a chloride ion (Fig. 6), forming a layer in the (001) plane. Two pairs of these twin chains surround an infinite

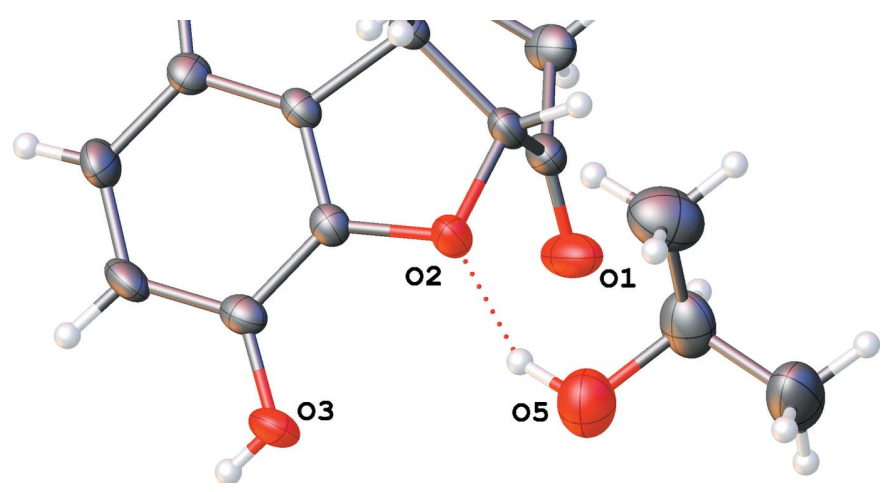

Figure 8

A dashed line indicates the $\mathrm{O}-\mathrm{H} \cdots \mathrm{O}$ hydrogen bond connecting a propan-2-ol molecule to an ether group of the naltrexone cation in (II). The minor component of the disordered propanol molecule is omitted for clarity. 
Table 3

Hydrogen-bond geometry $\left(\AA,^{\circ}\right)$ for (II).

\begin{tabular}{lllll}
\hline$D-\mathrm{H} \cdots A$ & $D-\mathrm{H}$ & $\mathrm{H} \cdots A$ & $D \cdots A$ & $D-\mathrm{H} \cdots A$ \\
\hline $\mathrm{N} 1-\mathrm{H} 1 \cdots \mathrm{Cl} 1^{\mathrm{i}}$ & $0.87(3)$ & $2.34(3)$ & $3.102(3)$ & $146(1)$ \\
$\mathrm{O} 3-\mathrm{H} 3 \cdots \mathrm{Cl}{ }^{\mathrm{ii}}$ & $0.75(4)$ & $2.32(4)$ & $3.066(3)$ & $169(4)$ \\
$\mathrm{O} 4-\mathrm{H} 4 \cdots \mathrm{Cl} 1$ & $0.85(4)$ & $2.21(4)$ & $3.054(2)$ & $177(3)$ \\
$\mathrm{O} 5-\mathrm{H} 5 A \cdots \mathrm{O} 2$ & $1.00(6)$ & $2.00(5)$ & $2.921(4)$ & $154(6)$ \\
\hline
\end{tabular}

Symmetry codes: (i) $x-\frac{1}{2},-y+\frac{1}{2},-z+1$; (ii) $-x+1, y+\frac{1}{2},-z+\frac{3}{2}$.

Table 4

Hydrogen-bond geometry $\left(\AA,^{\circ}\right)$ for (III).

\begin{tabular}{lllll}
\hline$D-\mathrm{H} \cdots A$ & $D-\mathrm{H}$ & $\mathrm{H} \cdots A$ & $D \cdots A$ & $D-\mathrm{H} \cdots A$ \\
\hline $\mathrm{N} 1-\mathrm{H} 1 \cdots \mathrm{Cl}{ }^{\mathrm{i}}$ & $0.99(3)$ & $2.43(3)$ & $3.245(3)$ & $140(3)$ \\
$\mathrm{O} 3-\mathrm{H} 3 \cdots \mathrm{Cl} 1$ & $0.84(4)$ & $2.20(3)$ & $2.999(2)$ & $162(2)$ \\
$\mathrm{O} 4-\mathrm{H} 4 \cdots \mathrm{C} 2^{\mathrm{ii}}$ & $0.86(3)$ & $2.21(3)$ & $3.063(2)$ & $175(1)$ \\
$\mathrm{O} 5-\mathrm{H} 5 A \cdots \mathrm{O} 2$ & $0.87(5)$ & $2.09(3)$ & $2.902(3)$ & $154(4)$ \\
$\mathrm{O} 6-\mathrm{H} 6 \cdots \mathrm{O} 5$ & 0.84 & 2.03 & $2.867(4)$ & 174 \\
$\mathrm{~N} 101-\mathrm{H} 101 \cdots \mathrm{Cl} 2^{\mathrm{iii}}$ & $0.84(2)$ & $2.57(2)$ & $3.239(3)$ & $138(2)$ \\
$\mathrm{O} 103-\mathrm{H} 103 \cdots \mathrm{Cl} 2$ & $0.87(4)$ & $2.15(3)$ & $3.002(2)$ & $164(3)$ \\
$\mathrm{O} 104-\mathrm{H} 104 \cdots \mathrm{Cl} 1$ & $0.87(3)$ & $2.16(3)$ & $3.026(2)$ & $175(1)$ \\
\hline
\end{tabular}

Symmetry codes: (i) $-x+1, y+\frac{1}{2},-z+1$; (ii) $x, y, z+1$; (iii) $-x, y-\frac{1}{2},-z$.

channel going along [010] axis containing the chloride ions and ethanol molecules (Fig. 7), thus forming a double layer in the (001) plane. These layers are bound to each other only by weak van der Waals interactions, despite the overall positive charge of the cation chains. The shortest contact involves an $\mathrm{O} 2$ oxygen atom of one layer and an $\mathrm{H} 15 A$ hydrogen atom of another, and has an $\mathrm{O}-\mathrm{H}$ separation of 2.60 (2) $\AA$, which is above threshold of hydrogen bonding.

In the propan-2-ol solvate (II), the alcohol molecule is also partially disordered. Both orientations make hydrogen bonds with ether oxygen atom, O2, of the dihydrofuran ring (Fig. 8). In this structure, a chloride ion is surrounded by two hydroxyl groups and the protonated nitrogen atom N1, all belonging to different naltrexone cations (Fig. 9, Table 3). These interactions result in a three-dimensional network, which has

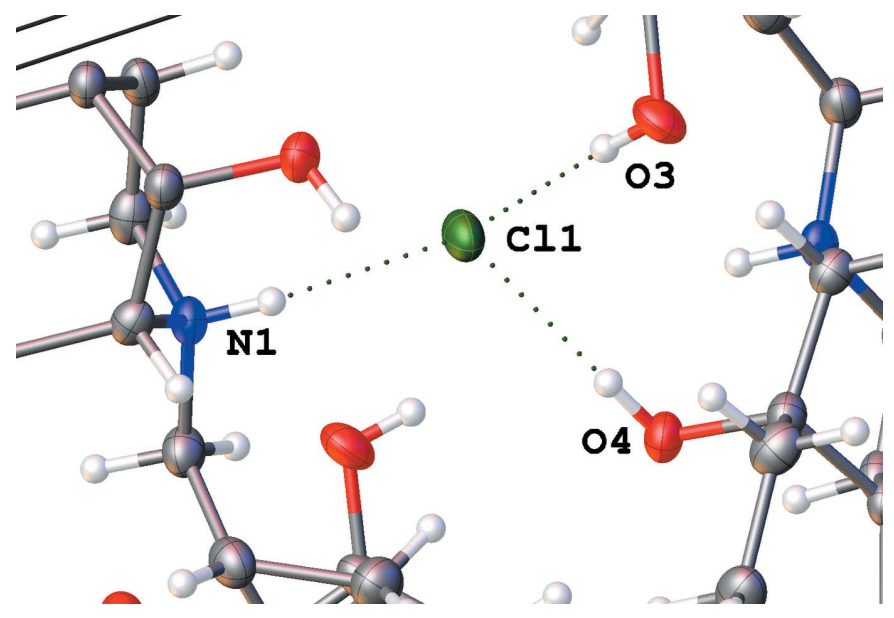

Figure 9

$\mathrm{N}-\mathrm{H} \cdots \mathrm{Cl}$ and $\mathrm{O}-\mathrm{H} \cdots \mathrm{Cl}$ hydrogen bonds around the chloride ion in the propan-2-ol solvate (II). Note that three different cations are connected.

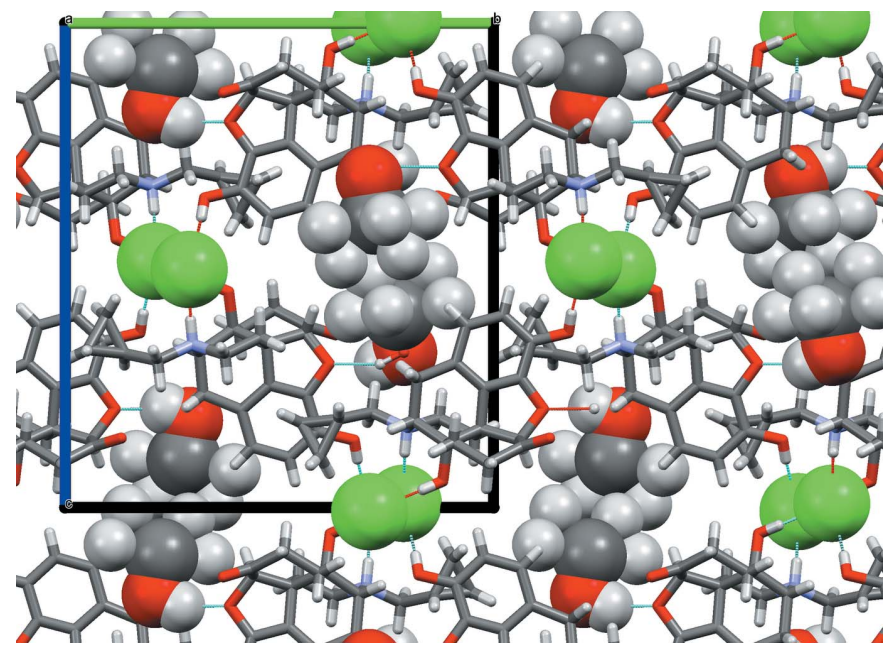

Figure 10

Packing diagram of the naltrexone ion associates in the propan-2-ol solvate (II), viewed along [100]. The chloride ions (green) and solvent molecules are highlighted.

solvent-filled infinite channels oriented along the [100] direction (Fig. 10).

In the 2-methylpropan-2-ol (tert-butanol) solvate (III), two tert-butanol molecules are connected via intermolecular hydrogen bonds; one of them makes a hydrogen bond to oxygen atom $\mathrm{O} 2$ (Fig. 11) of the naltrexone cation. The same hydroxyl group is located close to another oxygen atom, O3, but the $\mathrm{H} 5 A \cdots \mathrm{O} 3$ separation $(2.774 \AA$ ) is too long to be considered a real hydrogen bond. Another naltrexone cation in the same structure does not make direct hydrogen bonds to any solvate molecule. Similar to the propanol solvate, both crystallographically independent chloride ions are surrounded by two hydroxyl groups and protonated nitrogen atoms N1 and N101, all belonging to different naltrexone cations (Table 4). Again, the resulting three-dimensional network forms solvent-filled channels along the [100] direction (Fig. 12).

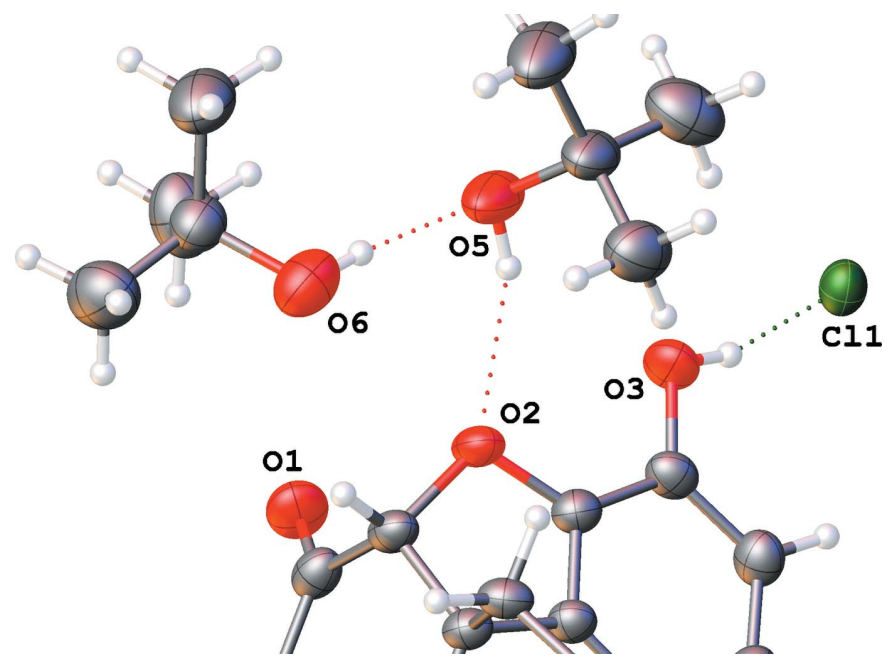

Figure 11

$\mathrm{O}-\mathrm{H} \cdots \mathrm{O}$ hydrogen bonds connecting the tert-butanol molecules in (III) to each other and to the ether group of a naltrexone cation. 


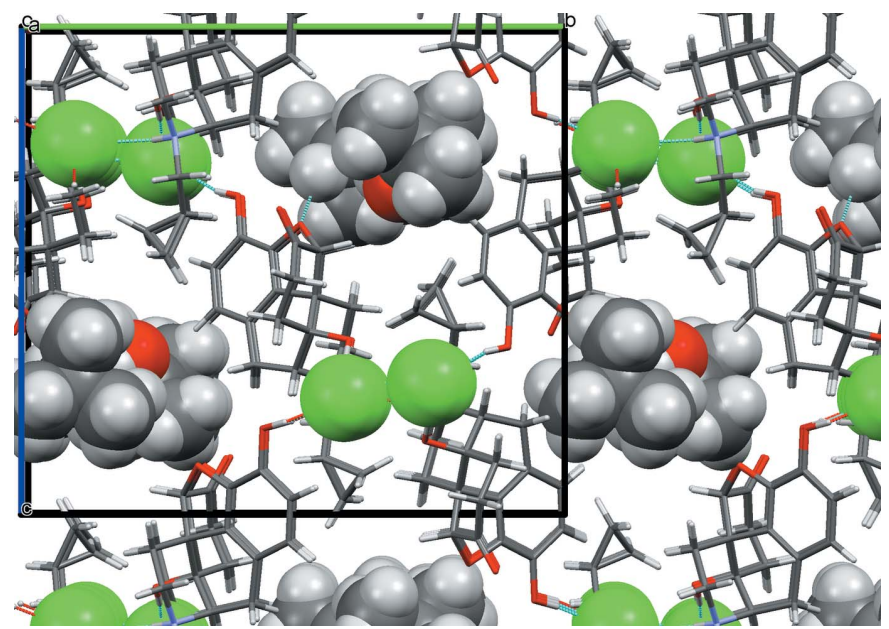

Figure 12

Packing diagram of the naltrexone ion associates in the 2-methylpropan2-ol (tert-butanol) solvate (III), viewed along [100]. The chloride ions (green) and solvent molecules are highlighted.

Contrary to the ethanol solvate, in the propanol and tertbutanol solvates, sequences of chloride ions occupy locations which are separate from the solvent-filled channels.

In the tetrahydrate (refcode PABCEA; Ledain et al., 1992) and formate hydrate (refcode YIGREM; Scheins et al., 2007), naltrexone cations form a chain via the protonated nitrogen atom and an oxygen atom of a carbonyl group, similar to what we see in the ethanol solvate. Water molecules and chloride ions also occupy a channel, this time along [001]. However, contrary to the ethanol solvate, the tetrahydrate structure does not exhibit a layered layout.

It is worth mentioning that in the ethanol solvate of oxymorphone hydrochloride (Darling et al., 1982), the ethanol molecule makes a weak hydrogen bond with the phenolic hydroxy group (atom $\mathrm{O} 3$ in our numbering scheme).

A plausible assumption is that interaction with an alcohol solvate molecule (or absence of it) does not affect significantly the structure of the naltrexone cation. Obviously, the presence of a strong hydrogen bond at the cyclohexanone carbonyl oxygen atom $\mathrm{O} 1$ (e.g., hydrate and ethanol solvate) is important; this affects the geometry of the cyclohexanone moiety and, possibly, the orientation of the methylcyclopropyl residue. Another significant factor is the size of a solvent-filled void. An increase of available space around the cyclopropylmethyl group may allow it to adopt a more favorable conformation.

\section{Database survey}

There are three reported naltrexone structures deposited in the Cambridge Structural Database (CSD Version 5.37; Groom et al., 2016). Of these, two report the structures of the chloride salt at room temperature (refcodes XINSAP and PABCEA), one of which (Sugimoto et al., 2007) is a powder structure of its anhydrous salt and the other (Ledain et al., 1992) a single-crystal investigation of tetrahydrate. A highquality charge-density investigation of the neutral naltrexone molecule and protonated naltrexone formate (refcodes YIGRAI and YIGREM; Scheins et al., 2007) was performed at $100 \mathrm{~K}$. A room-temperature structure of naltrexone malonate (refcode JEXRAF; Amato et al., 1990) is also known. The existence of various solvates of naltrexone chloride was reported from powder data (Nichols et al., 2013); however, no structural results were provided.

The crystal structure of oxymorphone hydrochloride monohydrate ethanol solvate (refcode BIZGAS) is also known (Darling et al., 1982). The experimental electrondensity distribution of naloxone hydrochloride dihydrate (refcode NALOXC02), another similar potent opiate antagonist, was described by Klein et al. (1987).

\section{Synthesis and crystallization}

Naltrexone hydrochloride (INTAS Ltd, India) was obtained as a mixture with lactose. The target compound was extracted from its starting form by recrystallization in ethanol, isopropanol, and tert-butanol. FTIR and Raman spectra of purified samples were consistent with database data for naltrexone hydrochloride. A GC-MS study showed one single peak on the chromatogram with $m / z: 341\left(M^{+}\right), 300\left(M-\mathrm{C}_{3} \mathrm{H}_{5}\right)$, $286\left(M-\mathrm{C}_{4} \mathrm{H}_{7}\right)$. A portion of the extracted naltrexone was then derivatized using pentafluoropropionic anhydride (PFPA), resulting in a corresponding dipentafluoropropionate $(m / z): 633(M), 592\left(M-\mathrm{C}_{3} \mathrm{H}_{5}\right), 486\left(M-\mathrm{C}_{3} \mathrm{~F}_{5} \mathrm{O}\right)$. This is consistent with the existence of two hydroxyl groups in the naltrexone molecule and confirms the correct chemical formula.

Nevertheless, diffractograms obtained from the crystallized material were all different from each other and from known naltrexone hydrochloride hydrate and naltrexone hydrochloride crystals (Ledain et al., 1992; Sugimoto et al., 2007; Nichols et al., 2013). The quality of some of the solvate crystals was sufficient for single crystal investigation. Herein we report the results obtained.

\section{Refinement}

Crystal data, data collection and structure refinement details are summarized in Table 5.

In the ethanol solvate (I), the solvent molecules are disordered with occupancies being approximately in a 2:1 ratio [0.66 (3):0.34 (3)]. Rigid body restrains (RIGU) were applied during refinement. In the propanol solvate (II), the occupancy of the minor component of a disordered solvent molecule is only 0.178 (9), which required additional constraints (EXYZ and EADP) on the position of the hydroxy group atoms. The tert-butanol solvate structure (III) was refined as a twocomponent twin (twin matrix: $\begin{array}{lllll}-1.000 & 0.000 & 0.000 & -0.001\end{array}$ $-1.0000 .0000 .1640 .0001 .000)$. There is visible flexibility in positions of the methyl groups of the tertiary tert-butanol molecules, which results in larger displacement parameters and could be potentially treated as disorder. However, we do not see the need for additional over-complication of the refinement procedure. 
Table 5

Experimental details.

Crystal data

Chemical formula

$M_{\mathrm{r}}$

Crystal system, space group

Temperature (K)

$a, b, c(\AA)$

$\alpha, \beta, \gamma\left({ }^{\circ}\right)$

$V\left(\AA^{3}\right)$

Z

Radiation type

$\mu\left(\mathrm{mm}^{-1}\right)$

Crystal size (mm)

Data collection

Diffractometer

Absorption correction

$T_{\min }, T_{\max }$

No. of measured, independent and observed $[I>2 \sigma(I)]$ reflections

$R_{\text {int }}$

$(\sin \theta / \lambda)_{\max }\left(\AA^{-1}\right)$

$\mathrm{C}_{20} \mathrm{H}_{24} \mathrm{NO}_{4}{ }^{+} \cdot \mathrm{Cl}^{-} \cdot \mathrm{C}_{2} \mathrm{H}_{6} \mathrm{O}$

423.92

Monoclinic, $P 2_{1}$

173

8.6885 (7), 7.9478 (6), 15.3417 (10)

1028.35 (13)

2

Mo $K \alpha$

0.22

$0.56 \times 0.13 \times 0.06$

Bruker PHOTON-100 CMOS

Multi-scan (SADABS; Bruker, 2015)

$0.891,1.000$

$33845,5866,5008$

0.042

0.700

Refinement

$R\left[F^{2}>2 \sigma\left(F^{2}\right)\right], w R\left(F^{2}\right), S$

No. of reflections

No. of parameters

No. of restraints

$\mathrm{H}$-atom treatment

$\Delta \rho_{\max }, \Delta \rho_{\min }\left(\mathrm{e} \AA^{-3}\right)$

Absolute structure

Absolute structure parameter -0.017 (18)
(I) (II)

90, 103.908 (2), 90

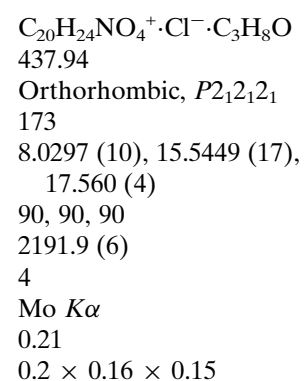

(III)

Bruker PHOTON-100 CMOS

Multi-scan ( $S A D A B S$; Bruker,

$0.925,0.986$

$48846,5327,4481$

0.665

$0.045,0.112,1.09$

5327

313

16

$0.038,0.082,1.03$
5866
305
10
$\mathrm{H}$ atoms treated by a mixture of
$\quad$ independent and constrained
$\quad$ refinement
$0.32,-0.24$
Flack $x$ determined using 2015
$\quad$ quotients $\left[\left(I^{+}\right)-\left(I^{-}\right)\right] /\left[\left(I^{+}\right)+\left(I^{-}\right)\right]$
$\quad$ (Parsons et al., 2013)
$0.2 \times 0.16 \times 0.15$ 2015)

0.043

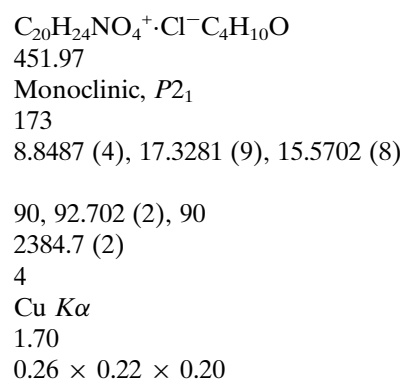

Bruker PHOTON-100 CMOS Multi-scan (TWINABS; Bruker, 2012)

$9642,9642,8820$

0.625

$0.033,0.079,1.04$

9642

581

1

$\mathrm{H}$ atoms treated by a mixture of independent and constrained refinement

$0.33,-0.34$

Flack $x$ determined using 1682 quotients $\left[\left(I^{+}\right)-\left(I^{-}\right)\right] /\left[\left(I^{+}\right)+\left(I^{-}\right)\right]$ (Parsons et al., 2013) $-0.028(18)$
$\mathrm{H}$ atoms treated by a mixture of independent and constrained refinement

$0.20,-0.17$

Flack $x$ determined using 3828 quotients $\left[\left(I^{+}\right)-\left(I^{-}\right)\right] /\left[\left(I^{+}\right)+\left(I^{-}\right)\right]$ (Parsons et al., 2013)

-0.004 (5)

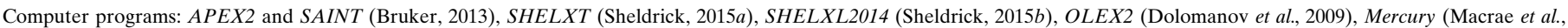
2006) and PLATON (Spek, 2009).

Hydrogen atoms of the hydroxyl groups were refined with riding coordinates and stretchable bonds. Hydrogen atoms of the protonated amine were refined isotropically or with riding coordinates and stretchable bonds, with $U_{\text {iso }}=1.2 U_{\text {iso }}(\mathrm{N})$ in all cases. All other hydrogen atoms were refined with riding coordinates, with $U_{\text {iso }}=1.5 U_{\text {iso }}(\mathrm{C})$ for methyl groups and $U_{\text {iso }}=1.2 U_{\text {iso }}(\mathrm{C})$ for all others.

\section{Acknowledgements}

Financial support from the State University of New York for acquisition and maintenance of the $\mathrm{X}$-ray diffractometer is gratefully acknowledged.

\section{References}

Amato, M. E., Bandoli, G., Grassi, A., Nicolini, M. \& Pappalardo, G. C. (1990). J. Chem. Soc. Perkin Trans. 2, pp. 1757-1762.

Bruker (2012). TWINABS. Bruker AXS Inc., Madison, Wisconsin, USA.

Bruker (2013). APEX2 and SAINT. Bruker AXS Inc., Madison, Wisconsin, USA.

Bruker (2015). SADABS. Bruker AXS Inc., Madison, Wisconsin, USA.
Compton, W. M. \& Volkow, N. D. (2006). Drug Alcohol Depend. 81, 103-107.

Darling, S. D., Kolb, V. M., Mandel, G. S. \& Mandel, N. S. (1982). J. Pharm. Sci. 71, 763-767.

Dolomanov, O. V., Bourhis, L. J., Gildea, R. J., Howard, J. A. K. \& Puschmann, H. (2009). J. Appl. Cryst. 42, 339-341.

Gauchat, E. \& Nazarenko, A. Y. (2017). Acta Cryst. E73, 63-67.

Gelbrich, T., Braun, D. E. \& Griesser, U. J. (2012). Acta Cryst. E68, o3358-o3359.

Groom, C. R., Bruno, I. J., Lightfoot, M. P. \& Ward, S. C. (2016). Acta Cryst. B72, 171-179.

Klein, C. L., Majeste, R. J. \& Stevens, E. D. (1987). J. Am. Chem. Soc. 109, 6675-6681.

Ledain, A. C., Madsen, B. W., Skelton, B. W. \& White, A. H. (1992). Aust. J. Chem. 45, 635-640.

Macrae, C. F., Edgington, P. R., McCabe, P., Pidcock, E., Shields, G. P., Taylor, R., Towler, M. \& van de Streek, J. (2006). J. Appl. Cryst. 39, 453-457.

Nichols, G. A., Menze, M. R. \& Mannino, A. (2013). US Patent US 8399475 B2.

Parsons, S., Flack, H. D. \& Wagner, T. (2013). Acta Cryst. B69, 249259.

Roozen, H. G., de Waart, R., van der Windt, D. A. W. M., van den Brink, W., de Jong, C. A. J. \& Kerkhof, A. J. F. M. (2006). Eur. Neuropsychopharmacol. 16, 311-323. 
Scheins, S., Messerschmidt, M., Morgenroth, W., Paulmann, C. \& Luger, P. (2007). J. Phys. Chem. A, 111, 5499-5508.

Sheldrick, G. M. (2015a). Acta Cryst. A71, 3-8.

Sheldrick, G. M. (2015b). Acta Cryst. C71, 3-8.
Spek, A. L. (2009). Acta Cryst. D65, 148-155.

Sugimoto, K., Dinnebier, R. E. \& Zakrzewski, M. (2007). J. Pharm. Sci. 96, 3316-3323. 


\section{supporting information}

Acta Cryst. (2017). E73, 1013-1020 [https://doi.org/10.1107/S205698901700843X]

Crystal structure of naltrexone chloride solvates with ethanol, propan-2-ol, and 2-methylpropan-2-ol

\section{Aveary R. Menze, Jefferson P. Sinnott and Alexander Y. Nazarenko}

Computing details

For all compounds, data collection: APEX2 (Bruker, 2013); cell refinement: SAINT (Bruker, 2013); data reduction: SAINT (Bruker, 2013); program(s) used to solve structure: SHELXT (Sheldrick, 2015a); program(s) used to refine structure: SHELXL2014 (Sheldrick, 2015b). Molecular graphics: OLEX2 (Dolomanov et al., 2009) and Mercury (Macrae et al., 2006) for (I); OLEX2 (Dolomanov et al., 2009) for (II), (III). Software used to prepare material for publication: OLEX2 (Dolomanov et al., 2009) and PLATON (Spek, 2009) for (I); OLEX2 (Dolomanov et al., 2009) for (II), (III).

(I) 17-(Cyclopropylmethyl)-3,14-dihydroxy-4,5a-epoxymorphinan-6-one hydrochloride ethanol monosolvate

Crystal data

$\mathrm{C}_{20} \mathrm{H}_{24} \mathrm{NO}_{4}{ }^{+} \cdot \mathrm{Cl}^{-} \cdot \mathrm{C}_{2} \mathrm{H}_{6} \mathrm{O}$

$M_{r}=423.92$

Monoclinic, $P 2_{1}$

$a=8.6885(7) \AA$

$b=7.9478(6) \AA$

$c=15.3417(10) \AA$

$\beta=103.908(2)^{\circ}$

$V=1028.35(13) \AA^{3}$

$Z=2$

Data collection

Bruker PHOTON-100 CMOS diffractometer

Radiation source: sealed tube $\varphi$ and $\omega$ scans

Absorption correction: multi-scan

(SADABS; Bruker, 2015)

$T_{\text {min }}=0.891, T_{\max }=1.000$

33845 measured reflections

Refinement

Refinement on $F^{2}$

Least-squares matrix: full

$R\left[F^{2}>2 \sigma\left(F^{2}\right)\right]=0.038$

$w R\left(F^{2}\right)=0.082$

$S=1.03$

5866 reflections

305 parameters

10 restraints

Hydrogen site location: mixed
$F(000)=452$

$D_{\mathrm{x}}=1.369 \mathrm{Mg} \mathrm{m}^{-3}$

Mo $K \alpha$ radiation, $\lambda=0.71073 \AA$

Cell parameters from 3107 reflections

$\theta=2.9-26.4^{\circ}$

$\mu=0.22 \mathrm{~mm}^{-1}$

$T=173 \mathrm{~K}$

Needle, colourless

$0.56 \times 0.13 \times 0.06 \mathrm{~mm}$

5866 independent reflections

5008 reflections with $I>2 \sigma(I)$

$R_{\text {int }}=0.042$

$\theta_{\text {max }}=29.9^{\circ}, \theta_{\min }=2.9^{\circ}$

$h=-12 \rightarrow 12$

$k=-10 \rightarrow 11$

$l=-21 \rightarrow 21$

$\mathrm{H}$ atoms treated by a mixture of independent and constrained refinement

$w=1 /\left[\sigma^{2}\left(F_{\mathrm{o}}^{2}\right)+(0.0403 P)^{2}+0.1718 P\right]$

where $P=\left(F_{\mathrm{o}}^{2}+2 F_{\mathrm{c}}^{2}\right) / 3$

$(\Delta / \sigma)_{\max }<0.001$

$\Delta \rho_{\max }=0.32 \mathrm{e} \AA^{-3}$

$\Delta \rho_{\min }=-0.24$ e $\AA^{-3}$ 
Absolute structure: Flack $x$ determined using

2015 quotients $\left[\left(I^{+}\right)-\left(I^{-}\right)\right] /\left[\left(I^{+}\right)+\left(I^{-}\right)\right]$(Parsons et al., 2013)

Absolute structure parameter: -0.017 (18)

\section{Special details}

Geometry. All esds (except the esd in the dihedral angle between two 1.s. planes) are estimated using the full covariance matrix. The cell esds are taken into account individually in the estimation of esds in distances, angles and torsion angles; correlations between esds in cell parameters are only used when they are defined by crystal symmetry. An approximate (isotropic) treatment of cell esds is used for estimating esds involving l.s. planes.

Fractional atomic coordinates and isotropic or equivalent isotropic displacement parameters $\left(\AA^{2}\right)$

\begin{tabular}{|c|c|c|c|c|c|}
\hline & $x$ & $y$ & $z$ & $U_{\text {iso }} * / U_{\text {eq }}$ & Occ. $(<1)$ \\
\hline $\mathrm{O} 1$ & 0.8998 (2) & $0.8845(2)$ & $0.08336(11)$ & $0.0259(4)$ & \\
\hline $\mathrm{O} 2$ & $0.64317(17)$ & $0.68560(19)$ & $0.04754(10)$ & $0.0203(3)$ & \\
\hline $\mathrm{O} 3$ & $0.42853(18)$ & $0.8802(2)$ & $0.12744(11)$ & $0.0223(3)$ & \\
\hline H3 & $0.401(3)$ & $0.943(3)$ & $0.1618(14)$ & $0.033^{*}$ & \\
\hline $\mathrm{O} 4$ & 1.03919 (19) & $0.29776(19)$ & $0.11509(10)$ & $0.0190(3)$ & \\
\hline $\mathrm{H} 4$ & $1.129(4)$ & $0.273(3)$ & 0.1404 (13) & $0.029 *$ & \\
\hline N1 & $0.8634(2)$ & $0.1391(2)$ & $0.20958(11)$ & $0.0163(3)$ & \\
\hline H1 & $0.923(3)$ & $0.101(3)$ & $0.1776(17)$ & $0.020 *$ & \\
\hline $\mathrm{C} 1$ & $0.6310(3)$ & $0.5970(3)$ & $0.31115(15)$ & $0.0210(5)$ & \\
\hline $\mathrm{H} 1 \mathrm{~A}$ & $0.6326(3)$ & $0.5760(7)$ & 0.3709 (19) & $0.025^{*}$ & \\
\hline $\mathrm{C} 2$ & $0.5273(3)$ & $0.7163(3)$ & $0.26364(15)$ & $0.0214(5)$ & \\
\hline $\mathrm{H} 2$ & $0.456(2)$ & $0.7737(17)$ & $0.2936(9)$ & $0.026^{*}$ & \\
\hline $\mathrm{C} 3$ & $0.5215(2)$ & $0.7569(3)$ & $0.17401(15)$ & $0.0190(4)$ & \\
\hline $\mathrm{C} 4$ & $0.6221(2)$ & $0.6658(3)$ & 0.13365 (13) & $0.0170(4)$ & \\
\hline $\mathrm{C} 5$ & $0.7949(2)$ & $0.6061(3)$ & 0.04998 (14) & $0.0170(4)$ & \\
\hline H5 & $0.7973(3)$ & $0.5645(12)$ & $-0.0074(16)$ & $0.020 *$ & \\
\hline C6 & $0.9275(3)$ & $0.7348(3)$ & $0.08214(14)$ & $0.0184(4)$ & \\
\hline $\mathrm{C} 7$ & $1.0886(3)$ & $0.6620(3)$ & $0.11905(15)$ & $0.0238(4)$ & \\
\hline H7A & $1.1658(16)$ & $0.7513(18)$ & 0.1360 (4) & $0.029 *$ & \\
\hline H7B & $1.1194(7)$ & $0.5929(14)$ & $0.0741(9)$ & $0.029^{*}$ & \\
\hline $\mathrm{C} 8$ & $1.0822(3)$ & $0.5547(3)$ & $0.20189(15)$ & $0.0192(4)$ & \\
\hline H8A & $1.186(2)$ & $0.5160(8)$ & $0.2306(6)$ & $0.023 *$ & \\
\hline H8B & $1.0434(8)$ & $0.6210(13)$ & $0.2440(8)$ & $0.023 *$ & \\
\hline C9 & $0.9473(2)$ & $0.2976(3)$ & $0.25188(13)$ & $0.0149(4)$ & \\
\hline H9 & $1.052(3)$ & $0.2645(9)$ & $0.2875(9)$ & $0.018 *$ & \\
\hline $\mathrm{C} 10$ & $0.8594(2)$ & $0.3851(3)$ & $0.31563(14)$ & $0.0177(4)$ & \\
\hline $\mathrm{H} 10 \mathrm{~A}$ & $0.8098(9)$ & $0.2985(16)$ & $0.3456(6)$ & $0.021 *$ & \\
\hline H10B & $0.9375(14)$ & $0.4451(11)$ & $0.3623(9)$ & $0.021 *$ & \\
\hline C11 & $0.7335(2)$ & $0.5080(3)$ & $0.26984(14)$ & $0.0165(4)$ & \\
\hline $\mathrm{C} 12$ & $0.7207(2)$ & $0.5434(3)$ & $0.18062(14)$ & $0.0157(4)$ & \\
\hline $\mathrm{C} 13$ & $0.8071(2)$ & $0.4592(3)$ & $0.11827(13)$ & $0.0144(4)$ & \\
\hline C14 & $0.9728(2)$ & $0.4048(3)$ & $0.17174(13)$ & $0.0145(4)$ & \\
\hline $\mathrm{C} 15$ & $0.7130(2)$ & $0.3023(3)$ & 0.07757 (14) & $0.0175(4)$ & \\
\hline H15A & $0.609(2)$ & $0.3349(7)$ & $0.0464(6)$ & $0.021 *$ & \\
\hline H15B & $0.7633(10)$ & $0.2520(10)$ & $0.0356(8)$ & $0.021^{*}$ & \\
\hline
\end{tabular}




\begin{tabular}{|c|c|c|c|c|c|}
\hline $\mathrm{C} 16$ & $0.7020(2)$ & $0.1746(3)$ & 0.14941 (14) & 0.0197 (4) & \\
\hline H16A & 0.6339 (13) & $0.2165(8)$ & $0.1845(7)$ & $0.024 *$ & \\
\hline H16B & $0.6575(9)$ & $0.0730(19)$ & 0.1217 (5) & $0.024^{*}$ & \\
\hline $\mathrm{C} 17$ & 0.8565 (3) & $-0.0007(3)$ & $0.27469(15)$ & $0.0217(5)$ & \\
\hline H17A & $0.7851(15)$ & $0.0308(7)$ & $0.3109(7)$ & $0.026^{*}$ & \\
\hline H17B & $0.8148(9)$ & $-0.100(2)$ & $0.2417(7)$ & $0.026^{*}$ & \\
\hline $\mathrm{C} 18$ & $1.0151(3)$ & $-0.0406(3)$ & 0.33484 (16) & $0.0248(5)$ & \\
\hline H18 & $1.100(3)$ & $-0.0555(5)$ & $0.3062(9)$ & $0.030^{*}$ & \\
\hline C19 & $1.0167(4)$ & $-0.1523(4)$ & 0.41391 (19) & $0.0374(6)$ & \\
\hline H19A & 0.917 (2) & $-0.1882(10)$ & $0.4233(3)$ & $0.045^{*}$ & \\
\hline H19B & $1.100(2)$ & $-0.233(2)$ & $0.4301(4)$ & $0.045^{*}$ & \\
\hline $\mathrm{C} 20$ & $1.0588(4)$ & 0.0293 (4) & $0.42825(17)$ & $0.0360(6)$ & \\
\hline $\mathrm{H} 20 \mathrm{~A}$ & $1.166(3)$ & $0.0567(8)$ & $0.4528(6)$ & $0.043 *$ & \\
\hline $\mathrm{H} 20 \mathrm{~B}$ & $0.9850(17)$ & 0.1009 (17) & $0.4460(5)$ & $0.043 *$ & \\
\hline $\mathrm{O} 5$ & $0.6227(2)$ & $1.1314(4)$ & $0.42038(13)$ & $0.0522(6)$ & \\
\hline $\mathrm{H} 5 \mathrm{~A}$ & 0.5552 & 1.1465 & 0.3717 & $0.078^{*}$ & $0.66(3)$ \\
\hline H5B & 0.5546 & 1.1413 & 0.3715 & $0.078^{*}$ & $0.34(3)$ \\
\hline $\mathrm{C} 21$ & $0.5677(11)$ & $1.0028(15)$ & $0.4713(7)$ & $0.046(2)$ & $0.66(3)$ \\
\hline $\mathrm{H} 21 \mathrm{~A}$ & $0.5584(11)$ & $0.888(5)$ & $0.4362(15)$ & $0.055^{*}$ & $0.66(3)$ \\
\hline $\mathrm{H} 21 \mathrm{~B}$ & $0.454(4)$ & $1.0348(19)$ & $0.4790(7)$ & $0.055^{*}$ & $0.66(3)$ \\
\hline $\mathrm{C} 21 \mathrm{~A}$ & $0.557(3)$ & $1.049(5)$ & 0.4808 (19) & $0.086(9)$ & $0.34(3)$ \\
\hline $\mathrm{H} 21 \mathrm{C}$ & 0.4644 & 0.9830 & 0.4484 & $0.103 *$ & $0.34(3)$ \\
\hline $\mathrm{H} 21 \mathrm{D}$ & 0.5208 & 1.1316 & 0.5196 & $0.103 *$ & $0.34(3)$ \\
\hline $\mathrm{C} 22$ & $0.6769(13)$ & $0.9820(15)$ & $0.5609(6)$ & 0.0408 (19) & $0.66(3)$ \\
\hline $\mathrm{H} 22 \mathrm{~A}$ & $0.781(4)$ & $0.955(5)$ & $0.5540(6)$ & $0.061 *$ & $0.66(3)$ \\
\hline $\mathrm{H} 22 \mathrm{~B}$ & $0.640(3)$ & $0.893(4)$ & $0.5925(16)$ & $0.061^{*}$ & $0.66(3)$ \\
\hline $\mathrm{H} 22 \mathrm{C}$ & $0.681(4)$ & $1.085(4)$ & $0.5942(15)$ & $0.061 *$ & $0.66(3)$ \\
\hline $\mathrm{C} 22 \mathrm{~A}$ & $0.673(3)$ & $0.937(3)$ & $0.5356(18)$ & $0.054(5)$ & $0.34(3)$ \\
\hline $\mathrm{H} 22 \mathrm{D}$ & 0.7009 & 0.8489 & 0.4978 & $0.081^{*}$ & $0.34(3)$ \\
\hline $\mathrm{H} 22 \mathrm{E}$ & 0.6272 & 0.8862 & 0.5819 & $0.081^{*}$ & $0.34(3)$ \\
\hline $\mathrm{H} 22 \mathrm{~F}$ & 0.7676 & 1.0014 & 0.5642 & $0.081^{*}$ & $0.34(3)$ \\
\hline Cl01 & $0.34066(7)$ & $1.14944(8)$ & $0.24667(4)$ & $0.03172(15)$ & \\
\hline
\end{tabular}

Atomic displacement parameters $\left(\AA^{2}\right)$

\begin{tabular}{lllllll}
\hline & $U^{11}$ & $U^{22}$ & $U^{33}$ & $U^{12}$ & $U^{13}$ & $U^{23}$ \\
\hline O1 & $0.0362(9)$ & $0.0179(8)$ & $0.0254(9)$ & $0.0005(7)$ & $0.0110(7)$ & $-0.0014(7)$ \\
O2 & $0.0210(7)$ & $0.0243(9)$ & $0.0158(7)$ & $0.0047(6)$ & $0.0047(6)$ & $0.0021(6)$ \\
O3 & $0.0208(8)$ & $0.0216(8)$ & $0.0240(8)$ & $0.0054(7)$ & $0.0046(6)$ & $-0.0015(7)$ \\
O4 & $0.0186(7)$ & $0.0218(8)$ & $0.0187(7)$ & $0.0034(6)$ & $0.0084(6)$ & $-0.0018(6)$ \\
N1 & $0.0202(8)$ & $0.0133(8)$ & $0.0168(8)$ & $-0.0008(7)$ & $0.0073(6)$ & $-0.0004(7)$ \\
C1 & $0.0236(11)$ & $0.0245(11)$ & $0.0165(10)$ & $0.0017(8)$ & $0.0081(9)$ & $-0.0008(8)$ \\
C2 & $0.0202(11)$ & $0.0233(11)$ & $0.0233(11)$ & $0.0021(9)$ & $0.0102(9)$ & $-0.0028(9)$ \\
C3 & $0.0148(10)$ & $0.0191(10)$ & $0.0225(10)$ & $-0.0004(8)$ & $0.0033(8)$ & $-0.0018(8)$ \\
C4 & $0.0165(9)$ & $0.0190(10)$ & $0.0154(9)$ & $-0.0019(8)$ & $0.0036(7)$ & $-0.0010(8)$ \\
C5 & $0.0203(10)$ & $0.0177(10)$ & $0.0138(9)$ & $0.0021(8)$ & $0.0059(8)$ & $0.0005(7)$ \\
C6 & $0.0257(11)$ & $0.0185(10)$ & $0.0141(10)$ & $-0.0019(8)$ & $0.0105(8)$ & $0.0008(8)$ \\
C7 & $0.0218(10)$ & $0.0198(10)$ & $0.0316(12)$ & $-0.0051(10)$ & $0.0100(9)$ & $0.0036(10)$
\end{tabular}




\begin{tabular}{lllllll} 
C8 & $0.0181(10)$ & $0.0180(10)$ & $0.0210(10)$ & $-0.0034(8)$ & $0.0035(8)$ & $0.0000(8)$ \\
C9 & $0.0160(9)$ & $0.0130(9)$ & $0.0155(9)$ & $-0.0013(8)$ & $0.0036(7)$ & $-0.0006(8)$ \\
C10 & $0.0217(10)$ & $0.0181(10)$ & $0.0138(9)$ & $0.0002(9)$ & $0.0054(8)$ & $-0.0001(8)$ \\
C11 & $0.0172(10)$ & $0.0170(10)$ & $0.0159(10)$ & $-0.0017(8)$ & $0.0051(8)$ & $-0.0020(8)$ \\
C12 & $0.0140(9)$ & $0.0158(10)$ & $0.0178(10)$ & $-0.0018(8)$ & $0.0050(8)$ & $-0.0027(8)$ \\
C13 & $0.0160(10)$ & $0.0145(10)$ & $0.0134(9)$ & $-0.0016(7)$ & $0.0050(7)$ & $-0.0005(7)$ \\
C14 & $0.0150(9)$ & $0.0153(10)$ & $0.0136(10)$ & $0.0004(7)$ & $0.0044(7)$ & $-0.0013(8)$ \\
C15 & $0.0178(10)$ & $0.0180(10)$ & $0.0156(9)$ & $-0.0019(8)$ & $0.0018(8)$ & $-0.0018(8)$ \\
C16 & $0.0192(10)$ & $0.0192(11)$ & $0.0204(10)$ & $-0.0052(8)$ & $0.0045(8)$ & $-0.0026(9)$ \\
C17 & $0.0313(12)$ & $0.0151(10)$ & $0.0210(10)$ & $-0.0046(9)$ & $0.0110(9)$ & $0.0013(9)$ \\
C18 & $0.0330(13)$ & $0.0196(11)$ & $0.0244(12)$ & $0.0040(9)$ & $0.0121(10)$ & $0.0034(9)$ \\
C19 & $0.0511(17)$ & $0.0309(15)$ & $0.0312(14)$ & $0.0082(12)$ & $0.0120(12)$ & $0.0132(11)$ \\
C20 & $0.0440(16)$ & $0.0339(14)$ & $0.0253(13)$ & $0.0051(12)$ & $-0.0008(11)$ & $0.0028(11)$ \\
O5 & $0.0357(10)$ & $0.0836(18)$ & $0.0374(11)$ & $0.0014(12)$ & $0.0089(8)$ & $0.0167(12)$ \\
C21 & $0.029(3)$ & $0.073(5)$ & $0.034(3)$ & $-0.009(4)$ & $0.002(3)$ & $0.000(3)$ \\
C21A & $0.068(10)$ & $0.131(18)$ & $0.076(13)$ & $0.042(10)$ & $0.054(9)$ & $0.059(13)$ \\
C22 & $0.037(3)$ & $0.060(5)$ & $0.025(3)$ & $-0.007(3)$ & $0.006(3)$ & $0.000(3)$ \\
C22A & $0.053(8)$ & $0.058(11)$ & $0.056(11)$ & $-0.003(8)$ & $0.023(9)$ & $0.008(8)$ \\
C101 & $0.0290(3)$ & $0.0350(3)$ & $0.0319(3)$ & $0.0134(3)$ & $0.0090(2)$ & $-0.0008(3)$ \\
& & & & & & \\
\hline
\end{tabular}

Geometric parameters $\left(\AA,{ }^{\circ}\right)$

\begin{tabular}{llll}
\hline $\mathrm{O} 1-\mathrm{C} 6$ & $1.215(3)$ & $\mathrm{C} 12-\mathrm{C} 13$ & $1.507(3)$ \\
$\mathrm{O} 2-\mathrm{C} 4$ & $1.386(2)$ & $\mathrm{C} 13-\mathrm{C} 14$ & $1.538(3)$ \\
$\mathrm{O} 2-\mathrm{C} 5$ & $1.454(2)$ & $\mathrm{C} 13-\mathrm{C} 15$ & $1.539(3)$ \\
$\mathrm{O} 3-\mathrm{H} 3$ & $0.80(3)$ & $\mathrm{C} 15-\mathrm{H} 15 \mathrm{~A}$ & $0.949(18)$ \\
$\mathrm{O} 3-\mathrm{C} 3$ & $1.359(3)$ & $\mathrm{C} 15-\mathrm{H} 15 \mathrm{~B}$ & $0.949(18)$ \\
$\mathrm{O} 4-\mathrm{H} 4$ & $0.81(3)$ & $\mathrm{C} 15-\mathrm{C} 16$ & $1.518(3)$ \\
$\mathrm{O} 4-\mathrm{C} 14$ & $1.433(2)$ & $\mathrm{C} 16-\mathrm{H} 16 \mathrm{~A}$ & $0.950(18)$ \\
$\mathrm{N} 1-\mathrm{H} 1$ & $0.85(3)$ & $\mathrm{C} 16-\mathrm{H} 16 \mathrm{~B}$ & $0.950(18)$ \\
$\mathrm{N} 1-\mathrm{C} 9$ & $1.521(3)$ & $\mathrm{C} 17-\mathrm{H} 17 \mathrm{~A}$ & $0.960(19)$ \\
$\mathrm{N} 1-\mathrm{C} 16$ & $1.509(3)$ & $\mathrm{C} 17-\mathrm{H} 17 \mathrm{~B}$ & $0.960(19)$ \\
$\mathrm{N} 1-\mathrm{C} 17$ & $1.505(3)$ & $\mathrm{C} 17-\mathrm{C} 18$ & $1.497(3)$ \\
$\mathrm{C} 1-\mathrm{H} 1 \mathrm{~A}$ & $0.93(3)$ & $\mathrm{C} 18-\mathrm{H} 18$ & $0.95(3)$ \\
$\mathrm{C} 1-\mathrm{C} 2$ & $1.388(3)$ & $\mathrm{C} 18-\mathrm{C} 19$ & $1.500(3)$ \\
$\mathrm{C} 1-\mathrm{C} 11$ & $\mathrm{C} 18-\mathrm{C} 20$ & $1.498(4)$ \\
$\mathrm{C} 2-\mathrm{H} 2$ & $1.402(3)$ & $\mathrm{C} 19-\mathrm{H} 19 \mathrm{~A}$ & $0.96(2)$ \\
$\mathrm{C} 2-\mathrm{C} 3$ & $0.97(3)$ & $\mathrm{C} 19-\mathrm{H} 19 \mathrm{~B}$ & $0.96(2)$ \\
$\mathrm{C} 3-\mathrm{C} 4$ & $1.402(3)$ & $\mathrm{C} 19-\mathrm{C} 20$ & $1.492(4)$ \\
$\mathrm{C} 4-\mathrm{C} 12$ & $1.390(3)$ & $\mathrm{C} 20-\mathrm{H} 20 \mathrm{~A}$ & $0.95(2)$ \\
$\mathrm{C} 5-\mathrm{H} 5$ & $1.379(3)$ & $\mathrm{C} 20-\mathrm{H} 20 \mathrm{~B}$ & $0.95(2)$ \\
$\mathrm{C} 5-\mathrm{C} 6$ & $0.95(3)$ & $\mathrm{O} 5-\mathrm{H} 5 \mathrm{~A}$ & 0.8400 \\
$\mathrm{C} 5-\mathrm{C} 13$ & $1.531(3)$ & $\mathrm{O} 5-\mathrm{H} 5 \mathrm{~B}$ & 0.8400 \\
$\mathrm{C} 6-\mathrm{C} 7$ & $1.555(3)$ & $\mathrm{O} 5-\mathrm{C} 21$ & $1.437(10)$ \\
$\mathrm{C} 7-\mathrm{H} 7 \mathrm{~A}$ & $1.494(3)$ & $\mathrm{O} 5-\mathrm{C} 21 \mathrm{~A}$ & $1.37(2)$ \\
$\mathrm{C} 7-\mathrm{H} 7 \mathrm{~B}$ & $0.968(19)$ & $\mathrm{C} 21-\mathrm{H} 21 \mathrm{~A}$ & $1.05(4)$ \\
$\mathrm{C} 7-\mathrm{C} 8$ & $0.968(19)$ & $\mathrm{C} 21-\mathrm{H} 21 \mathrm{~B}$ & $1.05(4)$ \\
$\mathrm{C} 8-\mathrm{H} 8 \mathrm{~A}$ & $1.543(3)$ & $\mathrm{C} 21-\mathrm{C} 22$ & $1.480(13)$ \\
& $0.956(18)$ & &
\end{tabular}




\begin{tabular}{|c|c|c|c|}
\hline $\mathrm{C} 8-\mathrm{H} 8 \mathrm{~B}$ & $0.956(18)$ & $\mathrm{C} 21 \mathrm{~A}-\mathrm{H} 21 \mathrm{C}$ & 0.9900 \\
\hline $\mathrm{C} 8-\mathrm{C} 14$ & $1.524(3)$ & $\mathrm{C} 21 \mathrm{~A}-\mathrm{H} 21 \mathrm{D}$ & 0.9900 \\
\hline C9-H9 & $0.98(2)$ & $\mathrm{C} 21 \mathrm{~A}-\mathrm{C} 22 \mathrm{~A}$ & $1.44(4)$ \\
\hline $\mathrm{C} 9-\mathrm{C} 10$ & $1.543(3)$ & $\mathrm{C} 22-\mathrm{H} 22 \mathrm{~A}$ & $0.96(3)$ \\
\hline $\mathrm{C} 9-\mathrm{C} 14$ & $1.555(3)$ & $\mathrm{C} 22-\mathrm{H} 22 \mathrm{~B}$ & $0.96(3)$ \\
\hline $\mathrm{C} 10-\mathrm{H} 10 \mathrm{~A}$ & $0.983(18)$ & $\mathrm{C} 22-\mathrm{H} 22 \mathrm{C}$ & $0.96(3)$ \\
\hline $\mathrm{C} 10-\mathrm{H} 10 \mathrm{~B}$ & $0.983(18)$ & $\mathrm{C} 22 \mathrm{~A}-\mathrm{H} 22 \mathrm{D}$ & 0.9800 \\
\hline $\mathrm{C} 10-\mathrm{C} 11$ & $1.509(3)$ & $\mathrm{C} 22 \mathrm{~A}-\mathrm{H} 22 \mathrm{E}$ & 0.9800 \\
\hline $\mathrm{C} 11-\mathrm{C} 12$ & $1.376(3)$ & $\mathrm{C} 22 \mathrm{~A}-\mathrm{H} 22 \mathrm{~F}$ & 0.9800 \\
\hline $\mathrm{C} 4-\mathrm{O} 2-\mathrm{C} 5$ & $104.64(15)$ & $\mathrm{O} 4-\mathrm{C} 14-\mathrm{C} 9$ & $107.99(16)$ \\
\hline $\mathrm{C} 3-\mathrm{O} 3-\mathrm{H} 3$ & 109.5 & $\mathrm{O} 4-\mathrm{C} 14-\mathrm{C} 13$ & $107.76(16)$ \\
\hline $\mathrm{C} 14-\mathrm{O} 4-\mathrm{H} 4$ & 109.5 & $\mathrm{C} 8-\mathrm{C} 14-\mathrm{C} 9$ & $112.67(16)$ \\
\hline $\mathrm{C} 9-\mathrm{N} 1-\mathrm{H} 1$ & $104.3(17)$ & $\mathrm{C} 8-\mathrm{C} 14-\mathrm{C} 13$ & $112.20(17)$ \\
\hline $\mathrm{C} 16-\mathrm{N} 1-\mathrm{H} 1$ & $108.6(17)$ & $\mathrm{C} 13-\mathrm{C} 14-\mathrm{C} 9$ & $106.39(16)$ \\
\hline $\mathrm{C} 16-\mathrm{N} 1-\mathrm{C} 9$ & $112.68(17)$ & $\mathrm{C} 13-\mathrm{C} 15-\mathrm{H} 15 \mathrm{~A}$ & 109.3 \\
\hline $\mathrm{C} 17-\mathrm{N} 1-\mathrm{H} 1$ & $104.2(17)$ & $\mathrm{C} 13-\mathrm{C} 15-\mathrm{H} 15 \mathrm{~B}$ & 109.3 \\
\hline $\mathrm{C} 17-\mathrm{N} 1-\mathrm{C} 9$ & $114.83(15)$ & $\mathrm{H} 15 \mathrm{~A}-\mathrm{C} 15-\mathrm{H} 15 \mathrm{~B}$ & 108.0 \\
\hline $\mathrm{C} 17-\mathrm{N} 1-\mathrm{C} 16$ & $111.38(16)$ & $\mathrm{C} 16-\mathrm{C} 15-\mathrm{C} 13$ & $111.55(17)$ \\
\hline $\mathrm{C} 2-\mathrm{C} 1-\mathrm{H} 1 \mathrm{~A}$ & 119.7 & $\mathrm{C} 16-\mathrm{C} 15-\mathrm{H} 15 \mathrm{~A}$ & 109.3 \\
\hline $\mathrm{C} 2-\mathrm{C} 1-\mathrm{C} 11$ & $120.7(2)$ & $\mathrm{C} 16-\mathrm{C} 15-\mathrm{H} 15 \mathrm{~B}$ & 109.3 \\
\hline $\mathrm{C} 11-\mathrm{C} 1-\mathrm{H} 1 \mathrm{~A}$ & 119.7 & $\mathrm{~N} 1-\mathrm{C} 16-\mathrm{C} 15$ & $110.95(16)$ \\
\hline $\mathrm{C} 1-\mathrm{C} 2-\mathrm{H} 2$ & 118.7 & $\mathrm{~N} 1-\mathrm{C} 16-\mathrm{H} 16 \mathrm{~A}$ & 109.4 \\
\hline $\mathrm{C} 1-\mathrm{C} 2-\mathrm{C} 3$ & $122.6(2)$ & $\mathrm{N} 1-\mathrm{C} 16-\mathrm{H} 16 \mathrm{~B}$ & 109.4 \\
\hline $\mathrm{C} 3-\mathrm{C} 2-\mathrm{H} 2$ & 118.7 & $\mathrm{C} 15-\mathrm{C} 16-\mathrm{H} 16 \mathrm{~A}$ & 109.4 \\
\hline $\mathrm{O} 3-\mathrm{C} 3-\mathrm{C} 2$ & $123.96(19)$ & $\mathrm{C} 15-\mathrm{C} 16-\mathrm{H} 16 \mathrm{~B}$ & 109.4 \\
\hline $\mathrm{O} 3-\mathrm{C} 3-\mathrm{C} 4$ & $119.97(19)$ & $\mathrm{H} 16 \mathrm{~A}-\mathrm{C} 16-\mathrm{H} 16 \mathrm{~B}$ & 108.0 \\
\hline $\mathrm{C} 4-\mathrm{C} 3-\mathrm{C} 2$ & $116.1(2)$ & $\mathrm{N} 1-\mathrm{C} 17-\mathrm{H} 17 \mathrm{~A}$ & 109.0 \\
\hline $\mathrm{O} 2-\mathrm{C} 4-\mathrm{C} 3$ & $127.38(19)$ & $\mathrm{N} 1-\mathrm{C} 17-\mathrm{H} 17 \mathrm{~B}$ & 109.0 \\
\hline $\mathrm{C} 12-\mathrm{C} 4-\mathrm{O} 2$ & $111.74(17)$ & $\mathrm{H} 17 \mathrm{~A}-\mathrm{C} 17-\mathrm{H} 17 \mathrm{~B}$ & 107.8 \\
\hline $\mathrm{C} 12-\mathrm{C} 4-\mathrm{C} 3$ & $120.84(19)$ & $\mathrm{C} 18-\mathrm{C} 17-\mathrm{N} 1$ & $112.82(18)$ \\
\hline $\mathrm{O} 2-\mathrm{C} 5-\mathrm{H} 5$ & 110.5 & $\mathrm{C} 18-\mathrm{C} 17-\mathrm{H} 17 \mathrm{~A}$ & 109.0 \\
\hline $\mathrm{O} 2-\mathrm{C} 5-\mathrm{C} 6$ & $109.01(16)$ & $\mathrm{C} 18-\mathrm{C} 17-\mathrm{H} 17 \mathrm{~B}$ & 109.0 \\
\hline $\mathrm{O} 2-\mathrm{C} 5-\mathrm{C} 13$ & $104.95(15)$ & $\mathrm{C} 17-\mathrm{C} 18-\mathrm{H} 18$ & 116.2 \\
\hline $\mathrm{C} 6-\mathrm{C} 5-\mathrm{H} 5$ & 110.5 & $\mathrm{C} 17-\mathrm{C} 18-\mathrm{C} 19$ & $116.9(2)$ \\
\hline $\mathrm{C} 6-\mathrm{C} 5-\mathrm{C} 13$ & $111.12(17)$ & $\mathrm{C} 17-\mathrm{C} 18-\mathrm{C} 20$ & $119.7(2)$ \\
\hline $\mathrm{C} 13-\mathrm{C} 5-\mathrm{H} 5$ & 110.5 & $\mathrm{C} 19-\mathrm{C} 18-\mathrm{H} 18$ & 116.2 \\
\hline $\mathrm{O} 1-\mathrm{C} 6-\mathrm{C} 5$ & $121.5(2)$ & $\mathrm{C} 20-\mathrm{C} 18-\mathrm{H} 18$ & 116.2 \\
\hline $\mathrm{O} 1-\mathrm{C} 6-\mathrm{C} 7$ & $123.0(2)$ & $\mathrm{C} 20-\mathrm{C} 18-\mathrm{C} 19$ & $59.68(18)$ \\
\hline $\mathrm{C} 7-\mathrm{C} 6-\mathrm{C} 5$ & $115.32(18)$ & $\mathrm{C} 18-\mathrm{C} 19-\mathrm{H} 19 \mathrm{~A}$ & 117.8 \\
\hline $\mathrm{C} 6-\mathrm{C} 7-\mathrm{H} 7 \mathrm{~A}$ & 110.1 & $\mathrm{C} 18-\mathrm{C} 19-\mathrm{H} 19 \mathrm{~B}$ & 117.8 \\
\hline $\mathrm{C} 6-\mathrm{C} 7-\mathrm{H} 7 \mathrm{~B}$ & 110.1 & $\mathrm{H} 19 \mathrm{~A}-\mathrm{C} 19-\mathrm{H} 19 \mathrm{~B}$ & 114.9 \\
\hline $\mathrm{C} 6-\mathrm{C} 7-\mathrm{C} 8$ & $108.08(17)$ & $\mathrm{C} 20-\mathrm{C} 19-\mathrm{C} 18$ & $60.09(17)$ \\
\hline $\mathrm{H} 7 \mathrm{~A}-\mathrm{C} 7-\mathrm{H} 7 \mathrm{~B}$ & 108.4 & $\mathrm{C} 20-\mathrm{C} 19-\mathrm{H} 19 \mathrm{~A}$ & 117.8 \\
\hline $\mathrm{C} 8-\mathrm{C} 7-\mathrm{H} 7 \mathrm{~A}$ & 110.1 & $\mathrm{C} 20-\mathrm{C} 19-\mathrm{H} 19 \mathrm{~B}$ & 117.8 \\
\hline $\mathrm{C} 8-\mathrm{C} 7-\mathrm{H} 7 \mathrm{~B}$ & 110.1 & $\mathrm{C} 18-\mathrm{C} 20-\mathrm{H} 20 \mathrm{~A}$ & 117.7 \\
\hline $\mathrm{C} 7-\mathrm{C} 8-\mathrm{H} 8 \mathrm{~A}$ & 109.9 & $\mathrm{C} 18-\mathrm{C} 20-\mathrm{H} 20 \mathrm{~B}$ & 117.7 \\
\hline $\mathrm{C} 7-\mathrm{C} 8-\mathrm{H} 8 \mathrm{~B}$ & 109.9 & $\mathrm{C} 19-\mathrm{C} 20-\mathrm{C} 18$ & $60.23(18)$ \\
\hline
\end{tabular}




\begin{tabular}{|c|c|c|c|}
\hline $\mathrm{H} 8 \mathrm{~A}-\mathrm{C} 8-\mathrm{H} 8 \mathrm{~B}$ & 108.3 & $\mathrm{C} 19-\mathrm{C} 20-\mathrm{H} 20 \mathrm{~A}$ & 117.7 \\
\hline $\mathrm{C} 14-\mathrm{C} 8-\mathrm{C} 7$ & $108.99(18)$ & $\mathrm{C} 19-\mathrm{C} 20-\mathrm{H} 20 \mathrm{~B}$ & 117.7 \\
\hline $\mathrm{C} 14-\mathrm{C} 8-\mathrm{H} 8 \mathrm{~A}$ & 109.9 & $\mathrm{H} 20 \mathrm{~A}-\mathrm{C} 20-\mathrm{H} 20 \mathrm{~B}$ & 114.9 \\
\hline $\mathrm{C} 14-\mathrm{C} 8-\mathrm{H} 8 \mathrm{~B}$ & 109.9 & $\mathrm{C} 21-\mathrm{O} 5-\mathrm{H} 5 \mathrm{~A}$ & 109.5 \\
\hline $\mathrm{N} 1-\mathrm{C} 9-\mathrm{H} 9$ & 107.5 & $\mathrm{C} 21 \mathrm{~A}-\mathrm{O} 5-\mathrm{H} 5 \mathrm{~B}$ & 109.5 \\
\hline $\mathrm{N} 1-\mathrm{C} 9-\mathrm{C} 10$ & $112.62(16)$ & $\mathrm{O} 5-\mathrm{C} 21-\mathrm{H} 21 \mathrm{~A}$ & 109.4 \\
\hline $\mathrm{N} 1-\mathrm{C} 9-\mathrm{C} 14$ & $105.16(15)$ & $\mathrm{O} 5-\mathrm{C} 21-\mathrm{H} 21 \mathrm{~B}$ & 109.4 \\
\hline $\mathrm{C} 10-\mathrm{C} 9-\mathrm{H} 9$ & 107.5 & $\mathrm{O} 5-\mathrm{C} 21-\mathrm{C} 22$ & $111.2(7)$ \\
\hline $\mathrm{C} 10-\mathrm{C} 9-\mathrm{C} 14$ & $116.16(17)$ & $\mathrm{H} 21 \mathrm{~A}-\mathrm{C} 21-\mathrm{H} 21 \mathrm{~B}$ & 108.0 \\
\hline $\mathrm{C} 14-\mathrm{C} 9-\mathrm{H} 9$ & 107.5 & $\mathrm{C} 22-\mathrm{C} 21-\mathrm{H} 21 \mathrm{~A}$ & 109.4 \\
\hline $\mathrm{C} 9-\mathrm{C} 10-\mathrm{H} 10 \mathrm{~A}$ & 108.7 & $\mathrm{C} 22-\mathrm{C} 21-\mathrm{H} 21 \mathrm{~B}$ & 109.4 \\
\hline C9- $\mathrm{C} 10-\mathrm{H} 10 \mathrm{~B}$ & 108.7 & $\mathrm{O} 5-\mathrm{C} 21 \mathrm{~A}-\mathrm{H} 21 \mathrm{C}$ & 109.6 \\
\hline $\mathrm{H} 10 \mathrm{~A}-\mathrm{C} 10-\mathrm{H} 10 \mathrm{~B}$ & 107.6 & $\mathrm{O} 5-\mathrm{C} 21 \mathrm{~A}-\mathrm{H} 21 \mathrm{D}$ & 109.6 \\
\hline $\mathrm{C} 11-\mathrm{C} 10-\mathrm{C} 9$ & $114.23(17)$ & $\mathrm{O} 5-\mathrm{C} 21 \mathrm{~A}-\mathrm{C} 22 \mathrm{~A}$ & $110(2)$ \\
\hline $\mathrm{C} 11-\mathrm{C} 10-\mathrm{H} 10 \mathrm{~A}$ & 108.7 & $\mathrm{H} 21 \mathrm{C}-\mathrm{C} 21 \mathrm{~A}-\mathrm{H} 21 \mathrm{D}$ & 108.1 \\
\hline $\mathrm{C} 11-\mathrm{C} 10-\mathrm{H} 10 \mathrm{~B}$ & 108.7 & $\mathrm{C} 22 \mathrm{~A}-\mathrm{C} 21 \mathrm{~A}-\mathrm{H} 21 \mathrm{C}$ & 109.6 \\
\hline $\mathrm{C} 1-\mathrm{C} 11-\mathrm{C} 10$ & $125.78(18)$ & $\mathrm{C} 22 \mathrm{~A}-\mathrm{C} 21 \mathrm{~A}-\mathrm{H} 21 \mathrm{D}$ & 109.6 \\
\hline $\mathrm{C} 12-\mathrm{C} 11-\mathrm{C} 1$ & $115.99(19)$ & $\mathrm{C} 21-\mathrm{C} 22-\mathrm{H} 22 \mathrm{~A}$ & 109.5 \\
\hline $\mathrm{C} 12-\mathrm{C} 11-\mathrm{C} 10$ & $118.16(18)$ & $\mathrm{C} 21-\mathrm{C} 22-\mathrm{H} 22 \mathrm{~B}$ & 109.5 \\
\hline $\mathrm{C} 4-\mathrm{C} 12-\mathrm{C} 13$ & $108.85(17)$ & $\mathrm{C} 21-\mathrm{C} 22-\mathrm{H} 22 \mathrm{C}$ & 109.5 \\
\hline $\mathrm{C} 11-\mathrm{C} 12-\mathrm{C} 4$ & $123.76(19)$ & $\mathrm{H} 22 \mathrm{~A}-\mathrm{C} 22-\mathrm{H} 22 \mathrm{~B}$ & 109.5 \\
\hline $\mathrm{C} 11-\mathrm{C} 12-\mathrm{C} 13$ & $127.38(19)$ & $\mathrm{H} 22 \mathrm{~A}-\mathrm{C} 22-\mathrm{H} 22 \mathrm{C}$ & 109.5 \\
\hline $\mathrm{C} 12-\mathrm{C} 13-\mathrm{C} 5$ & $97.49(16)$ & $\mathrm{H} 22 \mathrm{~B}-\mathrm{C} 22-\mathrm{H} 22 \mathrm{C}$ & 109.5 \\
\hline $\mathrm{C} 12-\mathrm{C} 13-\mathrm{C} 14$ & $109.36(16)$ & $\mathrm{C} 21 \mathrm{~A}-\mathrm{C} 22 \mathrm{~A}-\mathrm{H} 22 \mathrm{D}$ & 109.5 \\
\hline $\mathrm{C} 12-\mathrm{C} 13-\mathrm{C} 15$ & $108.69(16)$ & $\mathrm{C} 21 \mathrm{~A}-\mathrm{C} 22 \mathrm{~A}-\mathrm{H} 22 \mathrm{E}$ & 109.5 \\
\hline $\mathrm{C} 14-\mathrm{C} 13-\mathrm{C} 5$ & $118.28(16)$ & $\mathrm{C} 21 \mathrm{~A}-\mathrm{C} 22 \mathrm{~A}-\mathrm{H} 22 \mathrm{~F}$ & 109.5 \\
\hline $\mathrm{C} 14-\mathrm{C} 13-\mathrm{C} 15$ & $109.06(16)$ & $\mathrm{H} 22 \mathrm{D}-\mathrm{C} 22 \mathrm{~A}-\mathrm{H} 22 \mathrm{E}$ & 109.5 \\
\hline $\mathrm{C} 15-\mathrm{C} 13-\mathrm{C} 5$ & $113.06(17)$ & $\mathrm{H} 22 \mathrm{D}-\mathrm{C} 22 \mathrm{~A}-\mathrm{H} 22 \mathrm{~F}$ & 109.5 \\
\hline $\mathrm{O} 4-\mathrm{C} 14-\mathrm{C} 8$ & $109.61(16)$ & $\mathrm{H} 22 \mathrm{E}-\mathrm{C} 22 \mathrm{~A}-\mathrm{H} 22 \mathrm{~F}$ & 109.5 \\
\hline $\mathrm{O} 1-\mathrm{C} 6-\mathrm{C} 7-\mathrm{C} 8$ & $113.2(2)$ & $\mathrm{C} 6-\mathrm{C} 5-\mathrm{C} 13-\mathrm{C} 14$ & $-32.8(2)$ \\
\hline $\mathrm{O} 2-\mathrm{C} 4-\mathrm{C} 12-\mathrm{C} 11$ & $174.46(19)$ & $\mathrm{C} 6-\mathrm{C} 5-\mathrm{C} 13-\mathrm{C} 15$ & $-161.98(16)$ \\
\hline $\mathrm{O} 2-\mathrm{C} 4-\mathrm{C} 12-\mathrm{C} 13$ & $-5.8(2)$ & $\mathrm{C} 6-\mathrm{C} 7-\mathrm{C} 8-\mathrm{C} 14$ & $65.5(2)$ \\
\hline $\mathrm{O} 2-\mathrm{C} 5-\mathrm{C} 6-\mathrm{O} 1$ & $-15.5(3)$ & $\mathrm{C} 7-\mathrm{C} 8-\mathrm{C} 14-\mathrm{O} 4$ & $64.8(2)$ \\
\hline $\mathrm{O} 2-\mathrm{C} 5-\mathrm{C} 6-\mathrm{C} 7$ & $159.27(16)$ & $\mathrm{C} 7-\mathrm{C} 8-\mathrm{C} 14-\mathrm{C} 9$ & $-174.96(17)$ \\
\hline $\mathrm{O} 2-\mathrm{C} 5-\mathrm{C} 13-\mathrm{C} 12$ & $-33.70(19)$ & $\mathrm{C} 7-\mathrm{C} 8-\mathrm{C} 14-\mathrm{C} 13$ & $-54.9(2)$ \\
\hline $\mathrm{O} 2-\mathrm{C} 5-\mathrm{C} 13-\mathrm{C} 14$ & $-150.48(16)$ & $\mathrm{C} 9-\mathrm{N} 1-\mathrm{C} 16-\mathrm{C} 15$ & $55.4(2)$ \\
\hline $\mathrm{O} 2-\mathrm{C} 5-\mathrm{C} 13-\mathrm{C} 15$ & $80.3(2)$ & $\mathrm{C} 9-\mathrm{N} 1-\mathrm{C} 17-\mathrm{C} 18$ & $-51.0(2)$ \\
\hline $\mathrm{O} 3-\mathrm{C} 3-\mathrm{C} 4-\mathrm{O} 2$ & $0.7(3)$ & $\mathrm{C} 9-\mathrm{C} 10-\mathrm{C} 11-\mathrm{C} 1$ & $176.4(2)$ \\
\hline $\mathrm{O} 3-\mathrm{C} 3-\mathrm{C} 4-\mathrm{C} 12$ & $178.14(19)$ & $\mathrm{C} 9-\mathrm{C} 10-\mathrm{C} 11-\mathrm{C} 12$ & $-6.8(3)$ \\
\hline $\mathrm{N} 1-\mathrm{C} 9-\mathrm{C} 10-\mathrm{C} 11$ & $-87.3(2)$ & $\mathrm{C} 10-\mathrm{C} 9-\mathrm{C} 14-\mathrm{O} 4$ & $-173.55(16)$ \\
\hline $\mathrm{N} 1-\mathrm{C} 9-\mathrm{C} 14-\mathrm{O} 4$ & $-48.3(2)$ & $\mathrm{C} 10-\mathrm{C} 9-\mathrm{C} 14-\mathrm{C} 8$ & $65.3(2)$ \\
\hline $\mathrm{N} 1-\mathrm{C} 9-\mathrm{C} 14-\mathrm{C} 8$ & $-169.51(16)$ & $\mathrm{C} 10-\mathrm{C} 9-\mathrm{C} 14-\mathrm{C} 13$ & $-58.1(2)$ \\
\hline $\mathrm{N} 1-\mathrm{C} 9-\mathrm{C} 14-\mathrm{C} 13$ & $67.15(19)$ & $\mathrm{C} 10-\mathrm{C} 11-\mathrm{C} 12-\mathrm{C} 4$ & $-173.06(19)$ \\
\hline $\mathrm{N} 1-\mathrm{C} 17-\mathrm{C} 18-\mathrm{C} 19$ & $167.9(2)$ & $\mathrm{C} 10-\mathrm{C} 11-\mathrm{C} 12-\mathrm{C} 13$ & $7.3(3)$ \\
\hline $\mathrm{N} 1-\mathrm{C} 17-\mathrm{C} 18-\mathrm{C} 20$ & $99.2(3)$ & $\mathrm{C} 11-\mathrm{C} 1-\mathrm{C} 2-\mathrm{C} 3$ & $-1.5(3)$ \\
\hline $\mathrm{C} 1-\mathrm{C} 2-\mathrm{C} 3-\mathrm{O} 3$ & $-175.7(2)$ & $\mathrm{C} 11-\mathrm{C} 12-\mathrm{C} 13-\mathrm{C} 5$ & $-156.2(2)$ \\
\hline $\mathrm{C} 1-\mathrm{C} 2-\mathrm{C} 3-\mathrm{C} 4$ & $2.4(3)$ & $\mathrm{C} 11-\mathrm{C} 12-\mathrm{C} 13-\mathrm{C} 14$ & $-32.7(3)$ \\
\hline
\end{tabular}




$\begin{array}{ll}\mathrm{C} 1-\mathrm{C} 11-\mathrm{C} 12-\mathrm{C} 4 & 4.1(3) \\ \mathrm{C} 1-\mathrm{C} 11-\mathrm{C} 12-\mathrm{C} 13 & -175.5(2) \\ \mathrm{C} 2-\mathrm{C} 1-\mathrm{C} 11-\mathrm{C} 10 & 175.2(2) \\ \mathrm{C} 2-\mathrm{C} 1-\mathrm{C} 11-\mathrm{C} 12 & -1.7(3) \\ \mathrm{C} 2-\mathrm{C} 3-\mathrm{C} 4-\mathrm{O} 2 & -177.5(2) \\ \mathrm{C} 2-\mathrm{C} 3-\mathrm{C} 4-\mathrm{C} 12 & -0.1(3) \\ \mathrm{C} 3-\mathrm{C} 4-\mathrm{C} 12-\mathrm{C} 11 & -3.3(3) \\ \mathrm{C} 3-\mathrm{C} 4-\mathrm{C} 12-\mathrm{C} 13 & 176.38(19) \\ \mathrm{C} 4-\mathrm{O} 2-\mathrm{C} 5-\mathrm{C} 6 & -86.73(19) \\ \mathrm{C} 4-\mathrm{O} 2-\mathrm{C} 5-\mathrm{C} 13 & 32.4(2) \\ \mathrm{C} 4-\mathrm{C} 12-\mathrm{C} 13-\mathrm{C} 5 & 24.1(2) \\ \mathrm{C} 4-\mathrm{C} 12-\mathrm{C} 13-\mathrm{C} 14 & 147.62(17) \\ \mathrm{C} 4-\mathrm{C} 12-\mathrm{C} 13-\mathrm{C} 15 & -93.4(2) \\ \mathrm{C} 5-\mathrm{O} 2-\mathrm{C} 4-\mathrm{C} 3 & 160.5(2) \\ \mathrm{C} 5-\mathrm{O} 2-\mathrm{C} 4-\mathrm{C} 12 & -17.1(2) \\ \mathrm{C} 5-\mathrm{C} 6-\mathrm{C} 7-\mathrm{C} 8 & -61.5(2) \\ \mathrm{C} 5-\mathrm{C} 13-\mathrm{C} 14-\mathrm{O} 4 & -80.7(2) \\ \mathrm{C} 5-\mathrm{C} 13-\mathrm{C} 14-\mathrm{C} 8 & 40.0(2) \\ \mathrm{C} 5-\mathrm{C} 13-\mathrm{C} 14-\mathrm{C} 9 & 163.64(16) \\ \mathrm{C} 5-\mathrm{C} 13-\mathrm{C} 15-\mathrm{C} 16 & -169.37(17) \\ \mathrm{C} 6-\mathrm{C} 5-\mathrm{C} 13-\mathrm{C} 12 & 83.98(18)\end{array}$

$86.3(2)$

$169.02(16)$

$-70.2(2)$

$53.4(2)$

$-62.3(2)$

$-130.7(2)$

$44.1(2)$

$-50.5(2)$

$34.0(2)$

$56.9(2)$

$50.3(2)$

$171.03(17)$

$-65.34(19)$

$64.0(2)$

$-63.42(19)$

$179.37(18)$

$-65.0(2)$

$167.63(16)$

$-173.89(17)$

$-110.3(3)$

$105.6(3)$

Hydrogen-bond geometry $\left(\AA,{ }^{\circ}\right)$

\begin{tabular}{lllll}
\hline$D-\mathrm{H} \cdots A$ & $D-\mathrm{H}$ & $\mathrm{H} \cdots A$ & $D \cdots A$ & $D-\mathrm{H} \cdots A$ \\
\hline $\mathrm{N} 1-\mathrm{H} 1 \cdots \mathrm{O} 1^{\mathrm{i}}$ & $0.85(3)$ & $2.23(2)$ & $2.870(2)$ & $133(2)$ \\
$\mathrm{O} 3-\mathrm{H} 3 \cdots \mathrm{C} 101$ & $0.80(2)$ & $2.23(2)$ & $3.0297(17)$ & $171(2)$ \\
$\mathrm{O} 4-\mathrm{H} 4 \cdots \mathrm{C} 101^{\mathrm{ii}}$ & $0.81(3)$ & $2.36(3)$ & $3.1279(17)$ & $159(3)$ \\
$\mathrm{O} 5-\mathrm{H} 5 A \cdots \mathrm{C} 101$ & 0.84 & 2.33 & $3.160(2)$ & 169
\end{tabular}

Symmetry codes: (i) $x, y-1, z$; (ii) $x+1, y-1, z$.

(II) 17-(Cyclopropylmethyl)-3,14-dihydroxy-4,5a-epoxymorphinan-6-one hydrochloride propan-2-ol

monosolvate

Crystal data

$\mathrm{C}_{20} \mathrm{H}_{24} \mathrm{NO}_{4}{ }^{+} \cdot \mathrm{Cl}^{-} \cdot \mathrm{C}_{3} \mathrm{H}_{8} \mathrm{O}$

$M_{r}=437.94$

Orthorhombic, $P 2{ }_{1} 22_{1}$

$a=8.0297(10) \AA$

$b=15.5449(17) \AA$

$c=17.560(4) \AA$

$V=2191.9(6) \AA^{3}$

$Z=4$

$F(000)=936$

\section{Data collection}

Bruker PHOTON-100 CMOS

diffractometer

Radiation source: sealedtube
$D_{\mathrm{x}}=1.327 \mathrm{Mg} \mathrm{m}^{-3}$

Mo $K \alpha$ radiation, $\lambda=0.71073 \AA$

Cell parameters from 9935 reflections

$\theta=2.9-27.3^{\circ}$

$\mu=0.21 \mathrm{~mm}^{-1}$

$T=173 \mathrm{~K}$

Block, colourless

$0.2 \times 0.16 \times 0.15 \mathrm{~mm}$

$\varphi$ and $\omega$ scans

Absorption correction: multi-scan

(SADABS; Bruker, 2015) 
$T_{\min }=0.925, T_{\max }=0.986$

48846 measured reflections

5327 independent reflections

4481 reflections with $I>2 \sigma(I)$

$R_{\text {int }}=0.043$

\section{Refinement}

Refinement on $F^{2}$

Least-squares matrix: full

$R\left[F^{2}>2 \sigma\left(F^{2}\right)\right]=0.045$

$w R\left(F^{2}\right)=0.112$

$S=1.09$

5327 reflections

313 parameters

16 restraints

Primary atom site location: structure-invariant

direct methods

Hydrogen site location: mixed $\theta_{\max }=28.2^{\circ}, \theta_{\min }=2.8^{\circ}$

$h=-10 \rightarrow 10$

$k=-20 \rightarrow 19$

$l=-23 \rightarrow 22$

$\mathrm{H}$ atoms treated by a mixture of independent and constrained refinement

$w=1 /\left[\sigma^{2}\left(F_{\mathrm{o}}^{2}\right)+(0.0589 P)^{2}+0.6347 P\right]$ where $P=\left(F_{\mathrm{o}}^{2}+2 F_{\mathrm{c}}^{2}\right) / 3$

$(\Delta / \sigma)_{\max }<0.001$

$\Delta \rho_{\max }=0.33$ e $\AA^{-3}$

$\Delta \rho_{\min }=-0.34$ e $\AA^{-3}$

Absolute structure: Flack $x$ determined using 1682 quotients $\left[\left(I^{+}\right)-\left(I^{-}\right)\right] /\left[\left(I^{+}\right)+(I)\right]$ (Parsons et al., 2013)

Absolute structure parameter: -0.028 (18)

Special details

Geometry. All esds (except the esd in the dihedral angle between two 1.s. planes) are estimated using the full covariance matrix. The cell esds are taken into account individually in the estimation of esds in distances, angles and torsion angles; correlations between esds in cell parameters are only used when they are defined by crystal symmetry. An approximate (isotropic) treatment of cell esds is used for estimating esds involving l.s. planes.

Fractional atomic coordinates and isotropic or equivalent isotropic displacement parameters $\left(\AA^{2}\right)$

\begin{tabular}{|c|c|c|c|c|c|}
\hline & $x$ & $y$ & $z$ & $U_{\text {iso }} * / U_{\text {eq }}$ & Occ. $(<1)$ \\
\hline $\mathrm{Cl1}$ & $0.47836(9)$ & $0.21061(5)$ & $0.49145(4)$ & 0.02955 (19) & \\
\hline O1 & $0.6306(3)$ & $0.63086(16)$ & $0.64326(15)$ & $0.0468(7)$ & \\
\hline $\mathrm{O} 2$ & $0.3209(3)$ & $0.61657(13)$ & $0.70324(11)$ & $0.0291(5)$ & \\
\hline $\mathrm{O} 3$ & $0.3306(3)$ & $0.67460(13)$ & $0.86078(13)$ & $0.0334(5)$ & \\
\hline H3 & $0.366(6)$ & $0.6819(6)$ & $0.900(2)$ & $0.050 *$ & \\
\hline $\mathrm{O} 4$ & $0.3504(3)$ & $0.38140(14)$ & $0.55436(11)$ & $0.0264(5)$ & \\
\hline H4 & $0.390(4)$ & $0.335(3)$ & $0.5370(11)$ & $0.040 *$ & \\
\hline N1 & $0.1648(3)$ & $0.29790(15)$ & $0.66310(13)$ & $0.0211(5)$ & \\
\hline H1 & 0.1568 (4) & $0.2926(3)$ & $0.614(2)$ & $0.025^{*}$ & \\
\hline $\mathrm{C} 1$ & $0.3664(4)$ & $0.4365(2)$ & $0.87554(18)$ & $0.0340(7)$ & \\
\hline H1A & $0.3784(7)$ & $0.3966(19)$ & $0.9112(17)$ & $0.041 *$ & \\
\hline $\mathrm{C} 2$ & $0.3557(4)$ & $0.5228(2)$ & $0.89661(18)$ & $0.0332(7)$ & \\
\hline $\mathrm{H} 2$ & $0.3587(4)$ & $0.5365(6)$ & $0.947(2)$ & $0.040 *$ & \\
\hline $\mathrm{C} 3$ & $0.3405(4)$ & $0.58920(19)$ & $0.84301(17)$ & $0.0270(6)$ & \\
\hline $\mathrm{C} 4$ & $0.3334(4)$ & $0.56354(18)$ & $0.76731(16)$ & $0.0238(6)$ & \\
\hline $\mathrm{C} 5$ & $0.3665(4)$ & $0.56083(18)$ & $0.63904(16)$ & $0.0268(6)$ & \\
\hline $\mathrm{H} 5$ & 0.3063 & 0.5798 & 0.5921 & $0.032 *$ & \\
\hline C6 & $0.5551(4)$ & $0.5665(2)$ & $0.62618(17)$ & $0.0297(7)$ & \\
\hline $\mathrm{C} 7$ & $0.6358(4)$ & $0.4886(2)$ & $0.59096(19)$ & $0.0329(7)$ & \\
\hline $\mathrm{H} 7 \mathrm{~A}$ & $0.754(3)$ & $0.4958(3)$ & $0.59106(19)$ & $0.040^{*}$ & \\
\hline H7B & $0.6002(10)$ & $0.4832(2)$ & $0.5393(15)$ & $0.040^{*}$ & \\
\hline $\mathrm{C} 8$ & $0.5905(4)$ & $0.4063(2)$ & $0.6348(2)$ & $0.0297(7)$ & \\
\hline H8A & $0.6445(16)$ & $0.3595(13)$ & $0.6134(6)$ & $0.036^{*}$ & \\
\hline
\end{tabular}




\begin{tabular}{|c|c|c|c|c|c|}
\hline H8B & $0.6246(11)$ & $0.4112(2)$ & $0.6850(14)$ & $0.036^{*}$ & \\
\hline C9 & $0.3481(3)$ & 0.31327 (17) & $0.67995(16)$ & $0.0225(6)$ & \\
\hline H9 & $0.410(2)$ & $0.2633(18)$ & $0.6615(7)$ & $0.027 *$ & \\
\hline $\mathrm{C} 10$ & $0.3822(4)$ & $0.32180(19)$ & $0.76624(17)$ & $0.0281(6)$ & \\
\hline $\mathrm{H} 10 \mathrm{~A}$ & 0.3107 (19) & $0.2836(11)$ & $0.7926(7)$ & $0.034^{*}$ & \\
\hline H10B & $0.493(3)$ & $0.3042(5)$ & $0.7759(3)$ & $0.034^{*}$ & \\
\hline $\mathrm{C} 11$ & $0.3584(4)$ & 0.41151 (19) & $0.79858(16)$ & $0.0260(6)$ & \\
\hline $\mathrm{C} 12$ & $0.3361(4)$ & $0.47756(18)$ & $0.74739(15)$ & $0.0225(6)$ & \\
\hline $\mathrm{C} 13$ & $0.3061(3)$ & 0.46997 (18) & $0.66287(15)$ & $0.0221(6)$ & \\
\hline $\mathrm{C} 14$ & 0.4028 (4) & $0.39240(18)$ & $0.63174(16)$ & $0.0224(6)$ & \\
\hline $\mathrm{C} 15$ & $0.1182(4)$ & 0.45591 (18) & $0.64836(16)$ & $0.0241(6)$ & \\
\hline $\mathrm{H} 15 \mathrm{~A}$ & $0.0585(16)$ & $0.5021(12)$ & $0.6684(5)$ & $0.029 *$ & \\
\hline H15B & $0.0987(7)$ & $0.45401(18)$ & 0.5961 (14) & $0.029 *$ & \\
\hline $\mathrm{C} 16$ & 0.0577 (4) & 0.37308 (19) & $0.68449(17)$ & $0.0256(6)$ & \\
\hline H16A & 0.0577 (4) & $0.3791(2)$ & $0.7357(14)$ & $0.031^{*}$ & \\
\hline H16B & $-0.048(3)$ & $0.3628(3)$ & $0.6695(4)$ & $0.031^{*}$ & \\
\hline $\mathrm{C} 17$ & 0.0959 (4) & 0.21569 (19) & $0.69674(16)$ & $0.0268(6)$ & \\
\hline H17A & $0.0837(5)$ & $0.2231(3)$ & $0.7508(14)$ & $0.032 *$ & \\
\hline H17B & $-0.013(3)$ & $0.2055(3)$ & $0.6758(5)$ & $0.032 *$ & \\
\hline C18 & $0.2021(4)$ & $0.1386(2)$ & $0.68222(18)$ & $0.0295(7)$ & \\
\hline H18 & $0.304(5)$ & $0.1384(2)$ & $0.7058(11)$ & $0.035^{*}$ & \\
\hline $\mathrm{C} 19$ & $0.1196(4)$ & $0.0523(2)$ & $0.6740(2)$ & $0.0366(8)$ & \\
\hline H19A & $0.1726(17)$ & $0.0060(15)$ & $0.6936(7)$ & $0.044^{*}$ & \\
\hline H19B & $0.007(4)$ & $0.0503(2)$ & 0.6769 (2) & $0.044 *$ & \\
\hline $\mathrm{C} 20$ & 0.1953 (4) & $0.0949(2)$ & $0.6060(2)$ & $0.0338(7)$ & \\
\hline $\mathrm{H} 20 \mathrm{~A}$ & $0.126(2)$ & $0.1168(7)$ & 0.5707 (11) & $0.041^{*}$ & \\
\hline $\mathrm{H} 20 \mathrm{~B}$ & $0.290(3)$ & $0.0727(7)$ & $0.5874(6)$ & $0.041 *$ & \\
\hline O5 & 0.1923 (4) & $0.7903(2)$ & $0.67974(16)$ & $0.0566(7)$ & $0.822(9)$ \\
\hline $\mathrm{C} 21$ & $0.1900(8)$ & $0.7764(6)$ & $0.5993(3)$ & $0.0505(15)$ & $0.822(9)$ \\
\hline $\mathrm{H} 21$ & 0.2936 & 0.7453 & 0.5837 & $0.061 *$ & $0.822(9)$ \\
\hline C22 & $0.0361(8)$ & $0.7211(4)$ & 0.5788 (3) & $0.0660(17)$ & $0.822(9)$ \\
\hline $\mathrm{H} 22 \mathrm{~A}$ & 0.0335 & 0.7113 & 0.5237 & $0.099 *$ & $0.822(9)$ \\
\hline $\mathrm{H} 22 \mathrm{~B}$ & 0.0427 & 0.6658 & 0.6053 & $0.099^{*}$ & $0.822(9)$ \\
\hline $\mathrm{H} 22 \mathrm{C}$ & -0.0654 & 0.7514 & 0.5944 & $0.099 *$ & $0.822(9)$ \\
\hline $\mathrm{C} 23$ & $0.1858(10)$ & $0.8624(3)$ & 0.5619 (3) & $0.072(2)$ & $0.822(9)$ \\
\hline $\mathrm{H} 23 \mathrm{~A}$ & 0.1842 & 0.8551 & 0.5065 & $0.108^{*}$ & $0.822(9)$ \\
\hline $\mathrm{H} 23 \mathrm{~B}$ & 0.0857 & 0.8934 & 0.5780 & $0.108^{*}$ & $0.822(9)$ \\
\hline $\mathrm{H} 23 \mathrm{C}$ & 0.2849 & 0.8953 & 0.5766 & $0.108^{*}$ & $0.822(9)$ \\
\hline $\mathrm{H} 5 \mathrm{~A}$ & $0.225(9)$ & $0.735(3)$ & $0.704(4)$ & $0.108^{*}$ & $0.822(9)$ \\
\hline $\mathrm{O} 5 \mathrm{~A}$ & 0.1923 (4) & $0.7903(2)$ & $0.67974(16)$ & $0.0566(7)$ & $0.178(9)$ \\
\hline $\mathrm{C} 21 \mathrm{~A}$ & $0.141(4)$ & $0.779(3)$ & 0.6008 (12) & $0.0505(15)$ & $0.178(9)$ \\
\hline $\mathrm{H} 21 \mathrm{~A}$ & 0.1050 & 0.7173 & 0.5967 & $0.061^{*}$ & $0.178(9)$ \\
\hline $\mathrm{C} 22 \mathrm{~A}$ & -0.014 (4) & $0.832(3)$ & $0.5812(19)$ & $0.107(14)$ & $0.178(9)$ \\
\hline $\mathrm{H} 22 \mathrm{D}$ & -0.0443 & 0.8230 & 0.5278 & $0.160^{*}$ & $0.178(9)$ \\
\hline $\mathrm{H} 22 \mathrm{E}$ & -0.1066 & 0.8151 & 0.6141 & $0.160^{*}$ & $0.178(9)$ \\
\hline $\mathrm{H} 22 \mathrm{~F}$ & 0.0104 & 0.8936 & 0.5893 & $0.160^{*}$ & $0.178(9)$ \\
\hline $\mathrm{C} 23 \mathrm{~A}$ & $0.275(4)$ & $0.790(4)$ & $0.543(2)$ & $0.16(3)$ & $0.178(9)$ \\
\hline $\mathrm{H} 23 \mathrm{D}$ & 0.2281 & 0.7805 & 0.4917 & $0.242 *$ & $0.178(9)$ \\
\hline
\end{tabular}




\begin{tabular}{llllll}
$\mathrm{H} 23 \mathrm{E}$ & 0.3209 & 0.8478 & 0.5461 & $0.242^{*}$ & $0.178(9)$ \\
$\mathrm{H} 23 \mathrm{~F}$ & 0.3633 & 0.7474 & 0.5519 & $0.242^{*}$ & $0.178(9)$ \\
$\mathrm{H} 5 \mathrm{AA}$ & $0.225(9)$ & $0.735(3)$ & $0.704(4)$ & $0.242^{*}$ & $0.178(9)$ \\
\hline
\end{tabular}

Atomic displacement parameters $\left(\AA^{2}\right)$

\begin{tabular}{|c|c|c|c|c|c|c|}
\hline & $U^{11}$ & $U^{22}$ & $U^{\beta 3}$ & $U^{12}$ & $U^{13}$ & $U^{23}$ \\
\hline $\mathrm{C} 11$ & $0.0358(4)$ & $0.0311(3)$ & $0.0218(3)$ & $0.0075(3)$ & $0.0072(3)$ & $0.0006(3)$ \\
\hline $\mathrm{O} 1$ & $0.0546(16)$ & $0.0411(14)$ & $0.0448(15)$ & $-0.0170(12)$ & $0.0121(13)$ & $-0.0147(11)$ \\
\hline $\mathrm{O} 2$ & $0.0420(12)$ & $0.0249(10)$ & $0.0203(10)$ & $0.0035(9)$ & $0.0011(9)$ & $-0.0013(8)$ \\
\hline $\mathrm{O} 3$ & $0.0428(13)$ & $0.0330(11)$ & $0.0243(11)$ & $-0.0032(10)$ & $0.0005(10)$ & $-0.0113(9)$ \\
\hline $\mathrm{O} 4$ & $0.0321(11)$ & $0.0275(11)$ & $0.0196(10)$ & $0.0017(9)$ & $0.0008(9)$ & $-0.0048(8)$ \\
\hline N1 & $0.0197(11)$ & $0.0263(12)$ & $0.0171(11)$ & $-0.0015(10)$ & $-0.0018(9)$ & $-0.0030(9)$ \\
\hline $\mathrm{C} 1$ & $0.0434(19)$ & $0.0380(17)$ & $0.0207(15)$ & $-0.0049(15)$ & $-0.0086(14)$ & $0.0040(13)$ \\
\hline $\mathrm{C} 2$ & $0.0412(18)$ & 0.0409 (18) & $0.0174(15)$ & $-0.0099(15)$ & $-0.0029(13)$ & $-0.0066(12)$ \\
\hline $\mathrm{C} 3$ & $0.0257(14)$ & $0.0307(16)$ & $0.0246(15)$ & $-0.0044(12)$ & $0.0001(12)$ & $-0.0064(12)$ \\
\hline $\mathrm{C} 4$ & $0.0239(14)$ & $0.0270(14)$ & $0.0206(14)$ & $-0.0004(12)$ & $0.0002(12)$ & $-0.0005(11)$ \\
\hline $\mathrm{C} 5$ & $0.0381(16)$ & $0.0259(15)$ & $0.0163(13)$ & $0.0026(13)$ & $-0.0015(12)$ & $-0.0019(11)$ \\
\hline C6 & $0.0395(18)$ & $0.0301(15)$ & $0.0194(14)$ & $-0.0061(13)$ & $0.0013(12)$ & $-0.0021(12)$ \\
\hline $\mathrm{C} 7$ & $0.0295(16)$ & $0.0347(17)$ & $0.0347(17)$ & $-0.0055(13)$ & $0.0085(14)$ & $-0.0047(14)$ \\
\hline $\mathrm{C} 8$ & $0.0228(14)$ & $0.0294(16)$ & $0.0370(18)$ & $0.0026(12)$ & $0.0020(13)$ & $-0.0054(14)$ \\
\hline C9 & $0.0194(13)$ & $0.0230(14)$ & $0.0252(14)$ & $0.0018(11)$ & $-0.0017(11)$ & $-0.0036(11)$ \\
\hline C10 & $0.0314(16)$ & $0.0272(14)$ & $0.0259(15)$ & $0.0010(12)$ & $-0.0112(13)$ & $0.0041(12)$ \\
\hline C11 & $0.0255(14)$ & $0.0281(15)$ & $0.0244(14)$ & $-0.0031(12)$ & $-0.0061(12)$ & $-0.0007(12)$ \\
\hline $\mathrm{C} 12$ & $0.0209(13)$ & $0.0287(14)$ & $0.0179(13)$ & $-0.0007(12)$ & $-0.0018(11)$ & $-0.0028(11)$ \\
\hline $\mathrm{C} 13$ & $0.0238(14)$ & $0.0243(14)$ & $0.0181(13)$ & $0.0012(11)$ & $-0.0012(11)$ & $-0.0030(11)$ \\
\hline $\mathrm{C} 14$ & $0.0243(14)$ & $0.0243(14)$ & $0.0187(13)$ & $0.0023(11)$ & $-0.0010(11)$ & $-0.0028(11)$ \\
\hline $\mathrm{C} 15$ & $0.0265(15)$ & $0.0279(15)$ & $0.0180(13)$ & $0.0065(12)$ & $-0.0036(11)$ & $-0.0025(11)$ \\
\hline $\mathrm{C} 16$ & $0.0181(13)$ & $0.0345(16)$ & $0.0241(14)$ & $0.0033(11)$ & $-0.0006(11)$ & $-0.0073(12)$ \\
\hline $\mathrm{C} 17$ & $0.0299(15)$ & $0.0295(15)$ & $0.0211(14)$ & $-0.0040(13)$ & $0.0025(11)$ & $-0.0021(12)$ \\
\hline $\mathrm{C} 18$ & $0.0311(16)$ & $0.0322(16)$ & $0.0252(15)$ & $-0.0037(13)$ & $-0.0056(13)$ & $0.0023(13)$ \\
\hline C19 & $0.0325(17)$ & $0.0314(17)$ & $0.046(2)$ & $-0.0024(14)$ & $0.0065(15)$ & $0.0041(15)$ \\
\hline $\mathrm{C} 20$ & $0.0368(18)$ & $0.0321(17)$ & $0.0324(17)$ & $0.0032(14)$ & $0.0023(14)$ & $-0.0029(13)$ \\
\hline $\mathrm{O} 5$ & $0.0643(18)$ & $0.0561(16)$ & $0.0493(16)$ & $0.0071(15)$ & $0.0032(14)$ & $0.0064(14)$ \\
\hline $\mathrm{C} 21$ & $0.054(4)$ & $0.056(3)$ & $0.041(2)$ & $0.013(4)$ & $0.011(2)$ & 0.0018 (19) \\
\hline $\mathrm{C} 22$ & $0.065(3)$ & $0.067(4)$ & $0.066(3)$ & $0.006(3)$ & $-0.015(3)$ & $-0.011(3)$ \\
\hline $\mathrm{C} 23$ & $0.117(6)$ & $0.050(3)$ & $0.049(3)$ & $0.015(3)$ & $0.002(3)$ & $0.007(2)$ \\
\hline O5A & $0.0643(18)$ & $0.0561(16)$ & $0.0493(16)$ & $0.0071(15)$ & $0.0032(14)$ & $0.0064(14)$ \\
\hline $\mathrm{C} 21 \mathrm{~A}$ & $0.054(4)$ & $0.056(3)$ & $0.041(2)$ & $0.013(4)$ & $0.011(2)$ & 0.0018 (19) \\
\hline $\mathrm{C} 22 \mathrm{~A}$ & $0.089(15)$ & $0.14(3)$ & $0.09(2)$ & $0.052(18)$ & $0.023(14)$ & $0.02(2)$ \\
\hline C23A & $0.090(18)$ & $0.27(6)$ & $0.13(3)$ & $0.09(3)$ & $0.06(2)$ & $0.10(4)$ \\
\hline
\end{tabular}

Geometric parameters $\left(A,{ }^{o}\right)$

\begin{tabular}{llll}
\hline $\mathrm{O} 1-\mathrm{C} 6$ & $1.208(4)$ & $\mathrm{C} 15-\mathrm{H} 15 \mathrm{~A}$ & $0.93(2)$ \\
$\mathrm{O} 2-\mathrm{C} 4$ & $1.398(3)$ & $\mathrm{C} 15-\mathrm{H} 15 \mathrm{~B}$ & $0.93(2)$ \\
$\mathrm{O} 2-\mathrm{C} 5$ & $1.468(3)$ & $\mathrm{C} 15-\mathrm{C} 16$ & $1.515(4)$ \\
$\mathrm{O} 3-\mathrm{H} 3$ & $0.76(4)$ & $\mathrm{C} 16-\mathrm{H} 16 \mathrm{~A}$ & $0.90(2)$
\end{tabular}




\begin{tabular}{|c|c|c|c|}
\hline $\mathrm{O} 3-\mathrm{C} 3$ & $1.366(4)$ & $\mathrm{C} 16-\mathrm{H} 16 \mathrm{~B}$ & $0.90(2)$ \\
\hline $\mathrm{O} 4-\mathrm{H} 4$ & $0.84(4)$ & C17-H17A & $0.96(2)$ \\
\hline $\mathrm{O} 4-\mathrm{C} 14$ & $1.433(3)$ & C17-H17B & $0.96(2)$ \\
\hline $\mathrm{N} 1-\mathrm{H} 1$ & $0.86(3)$ & $\mathrm{C} 17-\mathrm{C} 18$ & $1.493(4)$ \\
\hline $\mathrm{N} 1-\mathrm{C} 9$ & $1.520(4)$ & $\mathrm{C} 18-\mathrm{H} 18$ & $0.91(4)$ \\
\hline $\mathrm{N} 1-\mathrm{C} 16$ & $1.499(4)$ & $\mathrm{C} 18-\mathrm{C} 19$ & $1.502(5)$ \\
\hline $\mathrm{N} 1-\mathrm{C} 17$ & $1.513(4)$ & $\mathrm{C} 18-\mathrm{C} 20$ & $1.502(5)$ \\
\hline $\mathrm{C} 1-\mathrm{H} 1 \mathrm{~A}$ & $0.89(4)$ & C19-H19A & $0.90(3)$ \\
\hline $\mathrm{C} 1-\mathrm{C} 2$ & $1.394(5)$ & C19-H19B & $0.90(3)$ \\
\hline $\mathrm{C} 1-\mathrm{C} 11$ & $1.408(4)$ & $\mathrm{C} 19-\mathrm{C} 20$ & $1.495(5)$ \\
\hline $\mathrm{C} 2-\mathrm{H} 2$ & $0.91(4)$ & $\mathrm{C} 20-\mathrm{H} 20 \mathrm{~A}$ & $0.90(3)$ \\
\hline $\mathrm{C} 2-\mathrm{C} 3$ & $1.403(5)$ & $\mathrm{C} 20-\mathrm{H} 20 \mathrm{~B}$ & $0.90(3)$ \\
\hline $\mathrm{C} 3-\mathrm{C} 4$ & $1.389(4)$ & $\mathrm{O} 5-\mathrm{C} 21$ & $1.429(6)$ \\
\hline $\mathrm{C} 4-\mathrm{C} 12$ & $1.382(4)$ & $\mathrm{O} 5-\mathrm{H} 5 \mathrm{~A}$ & 0.99 (3) \\
\hline C5-H5 & 1.0000 & $\mathrm{C} 21-\mathrm{H} 21$ & 1.0000 \\
\hline $\mathrm{C} 5-\mathrm{C} 6$ & $1.534(5)$ & $\mathrm{C} 21-\mathrm{C} 22$ & $1.547(10)$ \\
\hline $\mathrm{C} 5-\mathrm{C} 13$ & $1.551(4)$ & $\mathrm{C} 21-\mathrm{C} 23$ & $1.490(10)$ \\
\hline $\mathrm{C} 6-\mathrm{C} 7$ & $1.506(4)$ & $\mathrm{C} 22-\mathrm{H} 22 \mathrm{~A}$ & 0.9800 \\
\hline $\mathrm{C} 7-\mathrm{H} 7 \mathrm{~A}$ & $0.95(3)$ & $\mathrm{C} 22-\mathrm{H} 22 \mathrm{~B}$ & 0.9800 \\
\hline C7-H7B & $0.95(3)$ & $\mathrm{C} 22-\mathrm{H} 22 \mathrm{C}$ & 0.9800 \\
\hline $\mathrm{C} 7-\mathrm{C} 8$ & $1.537(5)$ & $\mathrm{C} 23-\mathrm{H} 23 \mathrm{~A}$ & 0.9800 \\
\hline $\mathrm{C} 8-\mathrm{H} 8 \mathrm{~A}$ & $0.93(3)$ & $\mathrm{C} 23-\mathrm{H} 23 \mathrm{~B}$ & 0.9800 \\
\hline $\mathrm{C} 8-\mathrm{H} 8 \mathrm{~B}$ & $0.93(3)$ & $\mathrm{C} 23-\mathrm{H} 23 \mathrm{C}$ & 0.9800 \\
\hline $\mathrm{C} 8-\mathrm{C} 14$ & $1.523(4)$ & $\mathrm{O} 5 \mathrm{~A}-\mathrm{C} 21 \mathrm{~A}$ & $1.46(2)$ \\
\hline C9-H9 & $0.98(3)$ & O5A-H5AA & $0.99(3)$ \\
\hline $\mathrm{C} 9-\mathrm{C} 10$ & $1.545(4)$ & $\mathrm{C} 21 \mathrm{~A}-\mathrm{H} 21 \mathrm{~A}$ & 1.0000 \\
\hline $\mathrm{C} 9-\mathrm{C} 14$ & $1.557(4)$ & $\mathrm{C} 21 \mathrm{~A}-\mathrm{C} 22 \mathrm{~A}$ & $1.54(3)$ \\
\hline $\mathrm{C} 10-\mathrm{H} 10 \mathrm{~A}$ & $0.95(3)$ & $\mathrm{C} 21 \mathrm{~A}-\mathrm{C} 23 \mathrm{~A}$ & $1.49(2)$ \\
\hline $\mathrm{C} 10-\mathrm{H} 10 \mathrm{~B}$ & $0.95(3)$ & $\mathrm{C} 22 \mathrm{~A}-\mathrm{H} 22 \mathrm{D}$ & 0.9800 \\
\hline $\mathrm{C} 10-\mathrm{C} 11$ & $1.518(4)$ & $\mathrm{C} 22 \mathrm{~A}-\mathrm{H} 22 \mathrm{E}$ & 0.9800 \\
\hline $\mathrm{C} 11-\mathrm{C} 12$ & $1.376(4)$ & $\mathrm{C} 22 \mathrm{~A}-\mathrm{H} 22 \mathrm{~F}$ & 0.9800 \\
\hline $\mathrm{C} 12-\mathrm{C} 13$ & $1.508(4)$ & $\mathrm{C} 23 \mathrm{~A}-\mathrm{H} 23 \mathrm{D}$ & 0.9800 \\
\hline $\mathrm{C} 13-\mathrm{C} 14$ & $1.535(4)$ & $\mathrm{C} 23 \mathrm{~A}-\mathrm{H} 23 \mathrm{E}$ & 0.9800 \\
\hline $\mathrm{C} 13-\mathrm{C} 15$ & $1.545(4)$ & $\mathrm{C} 23 \mathrm{~A}-\mathrm{H} 23 \mathrm{~F}$ & 0.9800 \\
\hline $\mathrm{C} 4-\mathrm{O} 2-\mathrm{C} 5$ & $104.6(2)$ & $\mathrm{C} 13-\mathrm{C} 15-\mathrm{H} 15 \mathrm{~B}$ & 109.4 \\
\hline $\mathrm{C} 3-\mathrm{O} 3-\mathrm{H} 3$ & 109.5 & $\mathrm{H} 15 \mathrm{~A}-\mathrm{C} 15-\mathrm{H} 15 \mathrm{~B}$ & 108.0 \\
\hline $\mathrm{C} 14-\mathrm{O} 4-\mathrm{H} 4$ & 109.5 & $\mathrm{C} 16-\mathrm{C} 15-\mathrm{C} 13$ & $111.4(2)$ \\
\hline $\mathrm{C} 9-\mathrm{N} 1-\mathrm{H} 1$ & 106.3 & $\mathrm{C} 16-\mathrm{C} 15-\mathrm{H} 15 \mathrm{~A}$ & 109.4 \\
\hline $\mathrm{C} 16-\mathrm{N} 1-\mathrm{H} 1$ & 106.3 & $\mathrm{C} 16-\mathrm{C} 15-\mathrm{H} 15 \mathrm{~B}$ & 109.4 \\
\hline $\mathrm{C} 16-\mathrm{N} 1-\mathrm{C} 9$ & $112.6(2)$ & $\mathrm{N} 1-\mathrm{C} 16-\mathrm{C} 15$ & $111.9(2)$ \\
\hline $\mathrm{C} 16-\mathrm{N} 1-\mathrm{C} 17$ & $110.6(2)$ & $\mathrm{N} 1-\mathrm{C} 16-\mathrm{H} 16 \mathrm{~A}$ & 109.2 \\
\hline $\mathrm{C} 17-\mathrm{N} 1-\mathrm{H} 1$ & 106.3 & $\mathrm{~N} 1-\mathrm{C} 16-\mathrm{H} 16 \mathrm{~B}$ & 109.2 \\
\hline $\mathrm{C} 17-\mathrm{N} 1-\mathrm{C} 9$ & $114.3(2)$ & $\mathrm{C} 15-\mathrm{C} 16-\mathrm{H} 16 \mathrm{~A}$ & 109.2 \\
\hline $\mathrm{C} 2-\mathrm{C} 1-\mathrm{H} 1 \mathrm{~A}$ & 119.4 & $\mathrm{C} 15-\mathrm{C} 16-\mathrm{H} 16 \mathrm{~B}$ & 109.2 \\
\hline $\mathrm{C} 2-\mathrm{C} 1-\mathrm{C} 11$ & $121.1(3)$ & $\mathrm{H} 16 \mathrm{~A}-\mathrm{C} 16-\mathrm{H} 16 \mathrm{~B}$ & 107.9 \\
\hline $\mathrm{C} 11-\mathrm{C} 1-\mathrm{H} 1 \mathrm{~A}$ & 119.4 & $\mathrm{~N} 1-\mathrm{C} 17-\mathrm{H} 17 \mathrm{~A}$ & 108.8 \\
\hline $\mathrm{C} 1-\mathrm{C} 2-\mathrm{H} 2$ & 118.8 & $\mathrm{~N} 1-\mathrm{C} 17-\mathrm{H} 17 \mathrm{~B}$ & 108.8 \\
\hline
\end{tabular}




\begin{tabular}{|c|c|c|c|}
\hline $\mathrm{C} 1-\mathrm{C} 2-\mathrm{C} 3$ & $122.4(3)$ & $\mathrm{H} 17 \mathrm{~A}-\mathrm{C} 17-\mathrm{H} 17 \mathrm{~B}$ & 107.7 \\
\hline $\mathrm{C} 3-\mathrm{C} 2-\mathrm{H} 2$ & 118.8 & $\mathrm{C} 18-\mathrm{C} 17-\mathrm{N} 1$ & $113.8(2)$ \\
\hline $\mathrm{O} 3-\mathrm{C} 3-\mathrm{C} 2$ & $124.6(3)$ & $\mathrm{C} 18-\mathrm{C} 17-\mathrm{H} 17 \mathrm{~A}$ & 108.8 \\
\hline $\mathrm{O} 3-\mathrm{C} 3-\mathrm{C} 4$ & $119.7(3)$ & $\mathrm{C} 18-\mathrm{C} 17-\mathrm{H} 17 \mathrm{~B}$ & 108.8 \\
\hline $\mathrm{C} 4-\mathrm{C} 3-\mathrm{C} 2$ & $115.7(3)$ & $\mathrm{C} 17-\mathrm{C} 18-\mathrm{H} 18$ & 115.7 \\
\hline $\mathrm{C} 3-\mathrm{C} 4-\mathrm{O} 2$ & $127.1(3)$ & $\mathrm{C} 17-\mathrm{C} 18-\mathrm{C} 19$ & $118.7(3)$ \\
\hline $\mathrm{C} 12-\mathrm{C} 4-\mathrm{O} 2$ & $111.6(2)$ & $\mathrm{C} 17-\mathrm{C} 18-\mathrm{C} 20$ & $119.6(3)$ \\
\hline $\mathrm{C} 12-\mathrm{C} 4-\mathrm{C} 3$ & $121.3(3)$ & $\mathrm{C} 19-\mathrm{C} 18-\mathrm{H} 18$ & 115.7 \\
\hline $\mathrm{O} 2-\mathrm{C} 5-\mathrm{H} 5$ & 109.8 & $\mathrm{C} 20-\mathrm{C} 18-\mathrm{H} 18$ & 115.7 \\
\hline $\mathrm{O} 2-\mathrm{C} 5-\mathrm{C} 6$ & $109.0(2)$ & $\mathrm{C} 20-\mathrm{C} 18-\mathrm{C} 19$ & $59.7(2)$ \\
\hline $\mathrm{O} 2-\mathrm{C} 5-\mathrm{C} 13$ & $104.6(2)$ & $\mathrm{C} 18-\mathrm{C} 19-\mathrm{H} 19 \mathrm{~A}$ & 117.8 \\
\hline $\mathrm{C} 6-\mathrm{C} 5-\mathrm{H} 5$ & 109.8 & C18-C19-H19B & 117.8 \\
\hline $\mathrm{C} 6-\mathrm{C} 5-\mathrm{C} 13$ & $113.6(2)$ & $\mathrm{H} 19 \mathrm{~A}-\mathrm{C} 19-\mathrm{H} 19 \mathrm{~B}$ & 114.9 \\
\hline $\mathrm{C} 13-\mathrm{C} 5-\mathrm{H} 5$ & 109.8 & $\mathrm{C} 20-\mathrm{C} 19-\mathrm{C} 18$ & $60.2(2)$ \\
\hline $\mathrm{O} 1-\mathrm{C} 6-\mathrm{C} 5$ & $120.4(3)$ & $\mathrm{C} 20-\mathrm{C} 19-\mathrm{H} 19 \mathrm{~A}$ & 117.8 \\
\hline $\mathrm{O} 1-\mathrm{C} 6-\mathrm{C} 7$ & $123.5(3)$ & $\mathrm{C} 20-\mathrm{C} 19-\mathrm{H} 19 \mathrm{~B}$ & 117.8 \\
\hline $\mathrm{C} 7-\mathrm{C} 6-\mathrm{C} 5$ & $116.1(3)$ & $\mathrm{C} 18-\mathrm{C} 20-\mathrm{H} 20 \mathrm{~A}$ & 117.8 \\
\hline $\mathrm{C} 6-\mathrm{C} 7-\mathrm{H} 7 \mathrm{~A}$ & 109.4 & $\mathrm{C} 18-\mathrm{C} 20-\mathrm{H} 20 \mathrm{~B}$ & 117.8 \\
\hline $\mathrm{C} 6-\mathrm{C} 7-\mathrm{H} 7 \mathrm{~B}$ & 109.4 & $\mathrm{C} 19-\mathrm{C} 20-\mathrm{C} 18$ & $60.2(2)$ \\
\hline $\mathrm{C} 6-\mathrm{C} 7-\mathrm{C} 8$ & $111.2(3)$ & $\mathrm{C} 19-\mathrm{C} 20-\mathrm{H} 20 \mathrm{~A}$ & 117.8 \\
\hline $\mathrm{H} 7 \mathrm{~A}-\mathrm{C} 7-\mathrm{H} 7 \mathrm{~B}$ & 108.0 & $\mathrm{C} 19-\mathrm{C} 20-\mathrm{H} 20 \mathrm{~B}$ & 117.8 \\
\hline $\mathrm{C} 8-\mathrm{C} 7-\mathrm{H} 7 \mathrm{~A}$ & 109.4 & $\mathrm{H} 20 \mathrm{~A}-\mathrm{C} 20-\mathrm{H} 20 \mathrm{~B}$ & 114.9 \\
\hline $\mathrm{C} 8-\mathrm{C} 7-\mathrm{H} 7 \mathrm{~B}$ & 109.4 & $\mathrm{C} 21-\mathrm{O} 5-\mathrm{H} 5 \mathrm{~A}$ & $107(4)$ \\
\hline $\mathrm{C} 7-\mathrm{C} 8-\mathrm{H} 8 \mathrm{~A}$ & 109.8 & $\mathrm{O} 5-\mathrm{C} 21-\mathrm{H} 21$ & 109.4 \\
\hline $\mathrm{C} 7-\mathrm{C} 8-\mathrm{H} 8 \mathrm{~B}$ & 109.8 & $\mathrm{O} 5-\mathrm{C} 21-\mathrm{C} 22$ & $108.9(5)$ \\
\hline $\mathrm{H} 8 \mathrm{~A}-\mathrm{C} 8-\mathrm{H} 8 \mathrm{~B}$ & 108.2 & $\mathrm{O} 5-\mathrm{C} 21-\mathrm{C} 23$ & $107.5(6)$ \\
\hline $\mathrm{C} 14-\mathrm{C} 8-\mathrm{C} 7$ & $109.5(3)$ & $\mathrm{C} 22-\mathrm{C} 21-\mathrm{H} 21$ & 109.4 \\
\hline $\mathrm{C} 14-\mathrm{C} 8-\mathrm{H} 8 \mathrm{~A}$ & 109.8 & $\mathrm{C} 23-\mathrm{C} 21-\mathrm{H} 21$ & 109.4 \\
\hline $\mathrm{C} 14-\mathrm{C} 8-\mathrm{H} 8 \mathrm{~B}$ & 109.8 & $\mathrm{C} 23-\mathrm{C} 21-\mathrm{C} 22$ & $112.2(5)$ \\
\hline $\mathrm{N} 1-\mathrm{C} 9-\mathrm{H} 9$ & 107.7 & $\mathrm{C} 21-\mathrm{C} 22-\mathrm{H} 22 \mathrm{~A}$ & 109.5 \\
\hline $\mathrm{N} 1-\mathrm{C} 9-\mathrm{C} 10$ & $112.1(2)$ & $\mathrm{C} 21-\mathrm{C} 22-\mathrm{H} 22 \mathrm{~B}$ & 109.5 \\
\hline $\mathrm{N} 1-\mathrm{C} 9-\mathrm{C} 14$ & $107.0(2)$ & $\mathrm{C} 21-\mathrm{C} 22-\mathrm{H} 22 \mathrm{C}$ & 109.5 \\
\hline $\mathrm{C} 10-\mathrm{C} 9-\mathrm{H} 9$ & 107.7 & $\mathrm{H} 22 \mathrm{~A}-\mathrm{C} 22-\mathrm{H} 22 \mathrm{~B}$ & 109.5 \\
\hline $\mathrm{C} 10-\mathrm{C} 9-\mathrm{C} 14$ & $114.5(2)$ & $\mathrm{H} 22 \mathrm{~A}-\mathrm{C} 22-\mathrm{H} 22 \mathrm{C}$ & 109.5 \\
\hline $\mathrm{C} 14-\mathrm{C} 9-\mathrm{H} 9$ & 107.7 & $\mathrm{H} 22 \mathrm{~B}-\mathrm{C} 22-\mathrm{H} 22 \mathrm{C}$ & 109.5 \\
\hline $\mathrm{C} 9-\mathrm{C} 10-\mathrm{H} 10 \mathrm{~A}$ & 108.5 & $\mathrm{C} 21-\mathrm{C} 23-\mathrm{H} 23 \mathrm{~A}$ & 109.5 \\
\hline C9- $\mathrm{C} 10-\mathrm{H} 10 \mathrm{~B}$ & 108.5 & $\mathrm{C} 21-\mathrm{C} 23-\mathrm{H} 23 \mathrm{~B}$ & 109.5 \\
\hline $\mathrm{H} 10 \mathrm{~A}-\mathrm{C} 10-\mathrm{H} 10 \mathrm{~B}$ & 107.5 & $\mathrm{C} 21-\mathrm{C} 23-\mathrm{H} 23 \mathrm{C}$ & 109.5 \\
\hline $\mathrm{C} 11-\mathrm{C} 10-\mathrm{C} 9$ & $115.0(2)$ & $\mathrm{H} 23 \mathrm{~A}-\mathrm{C} 23-\mathrm{H} 23 \mathrm{~B}$ & 109.5 \\
\hline $\mathrm{C} 11-\mathrm{C} 10-\mathrm{H} 10 \mathrm{~A}$ & 108.5 & $\mathrm{H} 23 \mathrm{~A}-\mathrm{C} 23-\mathrm{H} 23 \mathrm{C}$ & 109.5 \\
\hline $\mathrm{C} 11-\mathrm{C} 10-\mathrm{H} 10 \mathrm{~B}$ & 108.5 & $\mathrm{H} 23 \mathrm{~B}-\mathrm{C} 23-\mathrm{H} 23 \mathrm{C}$ & 109.5 \\
\hline $\mathrm{C} 1-\mathrm{C} 11-\mathrm{C} 10$ & $127.3(3)$ & $\mathrm{C} 21 \mathrm{~A}-\mathrm{O} 5 \mathrm{~A}-\mathrm{H} 5 \mathrm{AA}$ & $112(5)$ \\
\hline $\mathrm{C} 12-\mathrm{C} 11-\mathrm{C} 1$ & $115.3(3)$ & $\mathrm{O} 5 \mathrm{~A}-\mathrm{C} 21 \mathrm{~A}-\mathrm{H} 21 \mathrm{~A}$ & 105.5 \\
\hline $\mathrm{C} 12-\mathrm{C} 11-\mathrm{C} 10$ & $117.2(2)$ & $\mathrm{O} 5 \mathrm{~A}-\mathrm{C} 21 \mathrm{~A}-\mathrm{C} 22 \mathrm{~A}$ & $112(3)$ \\
\hline $\mathrm{C} 4-\mathrm{C} 12-\mathrm{C} 13$ & $108.8(2)$ & $\mathrm{O} 5 \mathrm{~A}-\mathrm{C} 21 \mathrm{~A}-\mathrm{C} 23 \mathrm{~A}$ & $116(3)$ \\
\hline $\mathrm{C} 11-\mathrm{C} 12-\mathrm{C} 4$ & $123.9(3)$ & $\mathrm{C} 22 \mathrm{~A}-\mathrm{C} 21 \mathrm{~A}-\mathrm{H} 21 \mathrm{~A}$ & 105.5 \\
\hline $\mathrm{C} 11-\mathrm{C} 12-\mathrm{C} 13$ & $127.2(3)$ & $\mathrm{C} 23 \mathrm{~A}-\mathrm{C} 21 \mathrm{~A}-\mathrm{H} 21 \mathrm{~A}$ & 105.5 \\
\hline $\mathrm{C} 12-\mathrm{C} 13-\mathrm{C} 5$ & $98.3(2)$ & $\mathrm{C} 23 \mathrm{~A}-\mathrm{C} 21 \mathrm{~A}-\mathrm{C} 22 \mathrm{~A}$ & $112(3)$ \\
\hline
\end{tabular}




\begin{tabular}{|c|c|c|c|}
\hline $\mathrm{C} 12-\mathrm{C} 13-\mathrm{C} 14$ & $109.3(2)$ & $\mathrm{C} 21 \mathrm{~A}-\mathrm{C} 22 \mathrm{~A}-\mathrm{H} 22 \mathrm{D}$ & 109.5 \\
\hline $\mathrm{C} 12-\mathrm{C} 13-\mathrm{C} 15$ & $109.2(2)$ & $\mathrm{C} 21 \mathrm{~A}-\mathrm{C} 22 \mathrm{~A}-\mathrm{H} 22 \mathrm{E}$ & 109.5 \\
\hline $\mathrm{C} 14-\mathrm{C} 13-\mathrm{C} 5$ & $117.4(2)$ & $\mathrm{C} 21 \mathrm{~A}-\mathrm{C} 22 \mathrm{~A}-\mathrm{H} 22 \mathrm{~F}$ & 109.5 \\
\hline $\mathrm{C} 14-\mathrm{C} 13-\mathrm{C} 15$ & $108.9(2)$ & $\mathrm{H} 22 \mathrm{D}-\mathrm{C} 22 \mathrm{~A}-\mathrm{H} 22 \mathrm{E}$ & 109.5 \\
\hline $\mathrm{C} 15-\mathrm{C} 13-\mathrm{C} 5$ & $112.9(2)$ & $\mathrm{H} 22 \mathrm{D}-\mathrm{C} 22 \mathrm{~A}-\mathrm{H} 22 \mathrm{~F}$ & 109.5 \\
\hline $\mathrm{O} 4-\mathrm{C} 14-\mathrm{C} 8$ & $109.9(3)$ & $\mathrm{H} 22 \mathrm{E}-\mathrm{C} 22 \mathrm{~A}-\mathrm{H} 22 \mathrm{~F}$ & 109.5 \\
\hline $\mathrm{O} 4-\mathrm{C} 14-\mathrm{C} 9$ & $109.8(2)$ & $\mathrm{C} 21 \mathrm{~A}-\mathrm{C} 23 \mathrm{~A}-\mathrm{H} 23 \mathrm{D}$ & 109.5 \\
\hline $\mathrm{O} 4-\mathrm{C} 14-\mathrm{C} 13$ & $106.4(2)$ & $\mathrm{C} 21 \mathrm{~A}-\mathrm{C} 23 \mathrm{~A}-\mathrm{H} 23 \mathrm{E}$ & 109.5 \\
\hline $\mathrm{C} 8-\mathrm{C} 14-\mathrm{C} 9$ & $111.9(2)$ & $\mathrm{C} 21 \mathrm{~A}-\mathrm{C} 23 \mathrm{~A}-\mathrm{H} 23 \mathrm{~F}$ & 109.5 \\
\hline $\mathrm{C} 8-\mathrm{C} 14-\mathrm{C} 13$ & $112.1(2)$ & $\mathrm{H} 23 \mathrm{D}-\mathrm{C} 23 \mathrm{~A}-\mathrm{H} 23 \mathrm{E}$ & 109.5 \\
\hline $\mathrm{C} 13-\mathrm{C} 14-\mathrm{C} 9$ & $106.5(2)$ & $\mathrm{H} 23 \mathrm{D}-\mathrm{C} 23 \mathrm{~A}-\mathrm{H} 23 \mathrm{~F}$ & 109.5 \\
\hline $\mathrm{C} 13-\mathrm{C} 15-\mathrm{H} 15 \mathrm{~A}$ & 109.4 & $\mathrm{H} 23 \mathrm{E}-\mathrm{C} 23 \mathrm{~A}-\mathrm{H} 23 \mathrm{~F}$ & 109.5 \\
\hline $\mathrm{O} 1-\mathrm{C} 6-\mathrm{C} 7-\mathrm{C} 8$ & $128.6(3)$ & $\mathrm{C} 6-\mathrm{C} 5-\mathrm{C} 13-\mathrm{C} 14$ & $-31.7(3)$ \\
\hline $\mathrm{O} 2-\mathrm{C} 4-\mathrm{C} 12-\mathrm{C} 11$ & $175.7(3)$ & $\mathrm{C} 6-\mathrm{C} 5-\mathrm{C} 13-\mathrm{C} 15$ & $-159.7(2)$ \\
\hline $\mathrm{O} 2-\mathrm{C} 4-\mathrm{C} 12-\mathrm{C} 13$ & $-6.0(3)$ & $\mathrm{C} 6-\mathrm{C} 7-\mathrm{C} 8-\mathrm{C} 14$ & $61.6(4)$ \\
\hline $\mathrm{O} 2-\mathrm{C} 5-\mathrm{C} 6-\mathrm{O} 1$ & $-28.1(4)$ & $\mathrm{C} 7-\mathrm{C} 8-\mathrm{C} 14-\mathrm{O} 4$ & $61.7(3)$ \\
\hline $\mathrm{O} 2-\mathrm{C} 5-\mathrm{C} 6-\mathrm{C} 7$ & $152.6(2)$ & $\mathrm{C} 7-\mathrm{C} 8-\mathrm{C} 14-\mathrm{C} 9$ & $-176.1(2)$ \\
\hline $\mathrm{O} 2-\mathrm{C} 5-\mathrm{C} 13-\mathrm{C} 12$ & $-33.5(3)$ & $\mathrm{C} 7-\mathrm{C} 8-\mathrm{C} 14-\mathrm{C} 13$ & $-56.5(3)$ \\
\hline $\mathrm{O} 2-\mathrm{C} 5-\mathrm{C} 13-\mathrm{C} 14$ & $-150.4(2)$ & $\mathrm{C} 9-\mathrm{N} 1-\mathrm{C} 16-\mathrm{C} 15$ & $53.8(3)$ \\
\hline $\mathrm{O} 2-\mathrm{C} 5-\mathrm{C} 13-\mathrm{C} 15$ & $81.5(3)$ & $\mathrm{C} 9-\mathrm{N} 1-\mathrm{C} 17-\mathrm{C} 18$ & $-47.9(3)$ \\
\hline $\mathrm{O} 3-\mathrm{C} 3-\mathrm{C} 4-\mathrm{O} 2$ & $1.8(5)$ & $\mathrm{C} 9-\mathrm{C} 10-\mathrm{C} 11-\mathrm{C} 1$ & $174.0(3)$ \\
\hline $\mathrm{O} 3-\mathrm{C} 3-\mathrm{C} 4-\mathrm{C} 12$ & $-177.3(3)$ & $\mathrm{C} 9-\mathrm{C} 10-\mathrm{C} 11-\mathrm{C} 12$ & $-10.2(4)$ \\
\hline $\mathrm{N} 1-\mathrm{C} 9-\mathrm{C} 10-\mathrm{C} 11$ & $-85.6(3)$ & $\mathrm{C} 10-\mathrm{C} 9-\mathrm{C} 14-\mathrm{O} 4$ & $-174.2(2)$ \\
\hline $\mathrm{N} 1-\mathrm{C} 9-\mathrm{C} 14-\mathrm{O} 4$ & $-49.3(3)$ & $\mathrm{C} 10-\mathrm{C} 9-\mathrm{C} 14-\mathrm{C} 8$ & $63.5(3)$ \\
\hline $\mathrm{N} 1-\mathrm{C} 9-\mathrm{C} 14-\mathrm{C} 8$ & $-171.7(2)$ & $\mathrm{C} 10-\mathrm{C} 9-\mathrm{C} 14-\mathrm{C} 13$ & $-59.3(3)$ \\
\hline $\mathrm{N} 1-\mathrm{C} 9-\mathrm{C} 14-\mathrm{C} 13$ & $65.5(3)$ & $\mathrm{C} 10-\mathrm{C} 11-\mathrm{C} 12-\mathrm{C} 4$ & $-171.7(3)$ \\
\hline $\mathrm{N} 1-\mathrm{C} 17-\mathrm{C} 18-\mathrm{C} 19$ & $-148.5(3)$ & $\mathrm{C} 10-\mathrm{C} 11-\mathrm{C} 12-\mathrm{C} 13$ & $10.2(4)$ \\
\hline $\mathrm{N} 1-\mathrm{C} 17-\mathrm{C} 18-\mathrm{C} 20$ & $-79.0(3)$ & $\mathrm{C} 11-\mathrm{C} 1-\mathrm{C} 2-\mathrm{C} 3$ & $-1.5(5)$ \\
\hline $\mathrm{C} 1-\mathrm{C} 2-\mathrm{C} 3-\mathrm{O} 3$ & $-179.6(3)$ & $\mathrm{C} 11-\mathrm{C} 12-\mathrm{C} 13-\mathrm{C} 5$ & $-157.5(3)$ \\
\hline $\mathrm{C} 1-\mathrm{C} 2-\mathrm{C} 3-\mathrm{C} 4$ & $1.2(5)$ & $\mathrm{C} 11-\mathrm{C} 12-\mathrm{C} 13-\mathrm{C} 14$ & $-34.5(4)$ \\
\hline $\mathrm{C} 1-\mathrm{C} 11-\mathrm{C} 12-\mathrm{C} 4$ & $4.6(5)$ & $\mathrm{C} 11-\mathrm{C} 12-\mathrm{C} 13-\mathrm{C} 15$ & $84.6(4)$ \\
\hline $\mathrm{C} 1-\mathrm{C} 11-\mathrm{C} 12-\mathrm{C} 13$ & $-173.5(3)$ & $\mathrm{C} 12-\mathrm{C} 13-\mathrm{C} 14-\mathrm{O} 4$ & $171.8(2)$ \\
\hline $\mathrm{C} 2-\mathrm{C} 1-\mathrm{C} 11-\mathrm{C} 10$ & $174.6(3)$ & $\mathrm{C} 12-\mathrm{C} 13-\mathrm{C} 14-\mathrm{C} 8$ & $-67.9(3)$ \\
\hline $\mathrm{C} 2-\mathrm{C} 1-\mathrm{C} 11-\mathrm{C} 12$ & $-1.3(5)$ & $\mathrm{C} 12-\mathrm{C} 13-\mathrm{C} 14-\mathrm{C} 9$ & $54.7(3)$ \\
\hline $\mathrm{C} 2-\mathrm{C} 3-\mathrm{C} 4-\mathrm{O} 2$ & $-179.0(3)$ & $\mathrm{C} 12-\mathrm{C} 13-\mathrm{C} 15-\mathrm{C} 16$ & $-61.9(3)$ \\
\hline $\mathrm{C} 2-\mathrm{C} 3-\mathrm{C} 4-\mathrm{C} 12$ & $2.0(4)$ & $\mathrm{C} 13-\mathrm{C} 5-\mathrm{C} 6-\mathrm{O} 1$ & $-144.3(3)$ \\
\hline $\mathrm{C} 3-\mathrm{C} 4-\mathrm{C} 12-\mathrm{C} 11$ & $-5.1(5)$ & $\mathrm{C} 13-\mathrm{C} 5-\mathrm{C} 6-\mathrm{C} 7$ & $36.4(4)$ \\
\hline $\mathrm{C} 3-\mathrm{C} 4-\mathrm{C} 12-\mathrm{C} 13$ & $173.2(3)$ & $\mathrm{C} 13-\mathrm{C} 15-\mathrm{C} 16-\mathrm{N} 1$ & $-50.9(3)$ \\
\hline $\mathrm{C} 4-\mathrm{O} 2-\mathrm{C} 5-\mathrm{C} 6$ & $-90.0(3)$ & $\mathrm{C} 14-\mathrm{C} 9-\mathrm{C} 10-\mathrm{C} 11$ & $36.5(4)$ \\
\hline $\mathrm{C} 4-\mathrm{O} 2-\mathrm{C} 5-\mathrm{C} 13$ & $31.9(3)$ & $\mathrm{C} 14-\mathrm{C} 13-\mathrm{C} 15-\mathrm{C} 16$ & $57.4(3)$ \\
\hline $\mathrm{C} 4-\mathrm{C} 12-\mathrm{C} 13-\mathrm{C} 5$ & $24.2(3)$ & $\mathrm{C} 15-\mathrm{C} 13-\mathrm{C} 14-\mathrm{O} 4$ & $52.5(3)$ \\
\hline $\mathrm{C} 4-\mathrm{C} 12-\mathrm{C} 13-\mathrm{C} 14$ & $147.2(2)$ & $\mathrm{C} 15-\mathrm{C} 13-\mathrm{C} 14-\mathrm{C} 8$ & $172.8(3)$ \\
\hline $\mathrm{C} 4-\mathrm{C} 12-\mathrm{C} 13-\mathrm{C} 15$ & $-93.7(3)$ & $\mathrm{C} 15-\mathrm{C} 13-\mathrm{C} 14-\mathrm{C} 9$ & $-64.5(3)$ \\
\hline $\mathrm{C} 5-\mathrm{O} 2-\mathrm{C} 4-\mathrm{C} 3$ & $164.1(3)$ & $\mathrm{C} 16-\mathrm{N} 1-\mathrm{C} 9-\mathrm{C} 10$ & $65.5(3)$ \\
\hline $\mathrm{C} 5-\mathrm{O} 2-\mathrm{C} 4-\mathrm{C} 12$ & $-16.7(3)$ & $\mathrm{C} 16-\mathrm{N} 1-\mathrm{C} 9-\mathrm{C} 14$ & $-60.9(3)$ \\
\hline $\mathrm{C} 5-\mathrm{C} 6-\mathrm{C} 7-\mathrm{C} 8$ & $-52.1(4)$ & $\mathrm{C} 16-\mathrm{N} 1-\mathrm{C} 17-\mathrm{C} 18$ & $-176.2(2)$ \\
\hline $\mathrm{C} 5-\mathrm{C} 13-\mathrm{C} 14-\mathrm{O} 4$ & $-77.4(3)$ & $\mathrm{C} 17-\mathrm{N} 1-\mathrm{C} 9-\mathrm{C} 10$ & $-61.7(3)$ \\
\hline
\end{tabular}




$\begin{array}{llll}\mathrm{C} 5-\mathrm{C} 13-\mathrm{C} 14-\mathrm{C} 8 & 42.9(4) & \mathrm{C} 17-\mathrm{N} 1-\mathrm{C} 9-\mathrm{C} 14 & 171.9(2) \\ \mathrm{C} 5-\mathrm{C} 13-\mathrm{C} 14-\mathrm{C} 9 & 165.5(2) & \mathrm{C} 17-\mathrm{N} 1-\mathrm{C} 16-\mathrm{C} 15 & -177.1(2) \\ \mathrm{C} 5-\mathrm{C} 13-\mathrm{C} 15-\mathrm{C} 16 & -170.2(2) & \mathrm{C} 17-\mathrm{C} 18-\mathrm{C} 19-\mathrm{C} 20 & 109.4(3) \\ \mathrm{C} 6-\mathrm{C} 5-\mathrm{C} 13-\mathrm{C} 12 & 85.2(3) & \mathrm{C} 17-\mathrm{C} 18-\mathrm{C} 20-\mathrm{C} 19 & -108.0(3)\end{array}$

Hydrogen-bond geometry $(\AA \stackrel{\circ}{\circ})$

\begin{tabular}{lllll}
\hline$D-\mathrm{H} \cdots A$ & $D-\mathrm{H}$ & $\mathrm{H} \cdots A$ & $D \cdots A$ & $D-\mathrm{H} \cdots A$ \\
\hline $\mathrm{N} 1-\mathrm{H} 1 \cdots \mathrm{C} 11^{\mathrm{i}}$ & $0.87(3)$ & $2.34(3)$ & $3.102(3)$ & $146(1)$ \\
$\mathrm{O} 3-\mathrm{H} 3 \cdots \mathrm{Cl1} 1^{\mathrm{ii}}$ & $0.75(4)$ & $2.32(4)$ & $3.066(3)$ & $169(4)$ \\
$\mathrm{O} 4-\mathrm{H} 4 \cdots \mathrm{C} 11$ & $0.85(4)$ & $2.21(4)$ & $3.054(2)$ & $177(3)$ \\
$\mathrm{O} 5-\mathrm{H} 5 A \cdots \mathrm{O} 2$ & $1.00(6)$ & $2.00(5)$ & $2.921(4)$ & $154(6)$ \\
\hline
\end{tabular}

Symmetry codes: (i) $x-1 / 2,-y+1 / 2,-z+1$; (ii) $-x+1, y+1 / 2,-z+3 / 2$.

(III) 17 -(Cyclopropylmethyl)-3,14-dihydroxy-4,5a-epoxymorphinan-6-one hydrochloride 2-methylpropan-2-ol monosolvate

Crystal data

$\mathrm{C}_{20} \mathrm{H}_{24} \mathrm{NO}_{4}{ }^{+} \cdot \mathrm{Cl}^{-} \mathrm{C}_{4} \mathrm{H}_{10} \mathrm{O}$

$M_{r}=451.97$

Monoclinic, $P 2_{1}$

$a=8.8487$ (4) $\AA$

$b=17.3281$ (9) $\AA$

$c=15.5702(8) \AA$

$\beta=92.702(2)^{\circ}$

$V=2384.7(2) \AA^{3}$

$Z=4$

\section{Data collection}

Bruker PHOTON-100 CMOS diffractometer

Radiation source: sealedtube $\varphi$ and $\omega$ scans

Absorption correction: multi-scan

(TWINABS; Bruker, 2012)
$F(000)=968$

$D_{\mathrm{x}}=1.259 \mathrm{Mg} \mathrm{m}^{-3}$

$\mathrm{Cu} K \alpha$ radiation, $\lambda=1.54178 \AA$

Cell parameters from 9282 reflections

$\theta=3.8-72.0^{\circ}$

$\mu=1.70 \mathrm{~mm}^{-1}$

$T=173 \mathrm{~K}$

Block, colourless

$0.26 \times 0.22 \times 0.20 \mathrm{~mm}$

9642 measured reflections

9642 independent reflections

8820 reflections with $I>2 \sigma(I)$

$\theta_{\text {max }}=74.6^{\circ}, \theta_{\text {min }}=2.8^{\circ}$

$h=-11 \rightarrow 11$

$k=-21 \rightarrow 21$

$l=0 \rightarrow 19$

$\mathrm{H}$ atoms treated by a mixture of independent and constrained refinement

$w=1 /\left[\sigma^{2}\left(F_{\mathrm{o}}^{2}\right)+(0.0369 P)^{2}+0.2646 P\right]$

where $P=\left(F_{\mathrm{o}}{ }^{2}+2 F_{\mathrm{c}}{ }^{2}\right) / 3$

$(\Delta / \sigma)_{\max }<0.001$

$\Delta \rho_{\max }=0.20 \mathrm{e} \AA^{-3}$

$\Delta \rho_{\min }=-0.17 \mathrm{e} \AA^{-3}$

Absolute structure: Flack $x$ determined using 3828 quotients $\left[\left(I^{+}\right)-(I)\right] /\left[\left(I^{+}\right)+\left(I^{-}\right)\right]$(Parsons et al., 2013)

Absolute structure parameter: -0.004 (5) 


\section{Special details}

Geometry. All esds (except the esd in the dihedral angle between two 1.s. planes) are estimated using the full covariance matrix. The cell esds are taken into account individually in the estimation of esds in distances, angles and torsion angles; correlations between esds in cell parameters are only used when they are defined by crystal symmetry. An approximate (isotropic) treatment of cell esds is used for estimating esds involving l.s. planes.

Refinement. Refined as a 2-component twin.

Fractional atomic coordinates and isotropic or equivalent isotropic displacement parameters $\left(\hat{A}^{2}\right)$

\begin{tabular}{|c|c|c|c|c|}
\hline & $x$ & $y$ & $z$ & $U_{\text {iso }} * / U_{\text {eq }}$ \\
\hline $\mathrm{C} 11$ & $0.66572(8)$ & $0.25519(4)$ & $0.26921(4)$ & $0.03945(16)$ \\
\hline $\mathrm{Cl} 2$ & $-0.15527(8)$ & $0.59450(4)$ & $-0.23882(4)$ & $0.03721(15)$ \\
\hline O101 & $0.4508(3)$ & $0.37330(15)$ & $-0.11407(14)$ & $0.0567(6)$ \\
\hline O102 & $0.1647(3)$ & $0.32376(12)$ & $-0.09967(11)$ & $0.0398(5)$ \\
\hline O103 & $-0.0224(3)$ & $0.44419(12)$ & $-0.17614(12)$ & $0.0409(5)$ \\
\hline H103 & $-0.077(5)$ & $0.485(2)$ & $-0.1889(6)$ & $0.061 *$ \\
\hline O104 & $0.3849(2)$ & 0.24407 (11) & $0.15125(12)$ & $0.0363(4)$ \\
\hline H104 & $0.466(4)$ & $0.2502(3)$ & $0.184(2)$ & $0.054 *$ \\
\hline N101 & 0.1251 & $0.28034(14)$ & $0.22373(13)$ & $0.0325(5)$ \\
\hline H101 & $0.182(2)$ & $0.2418(16)$ & $0.2306(3)$ & $0.039 *$ \\
\hline C101 & $-0.0057(4)$ & $0.49220(17)$ & $0.05622(18)$ & $0.0392(7)$ \\
\hline $\mathrm{H} 10 \mathrm{~A}$ & -0.0398 & 0.5315 & 0.0932 & $0.047^{*}$ \\
\hline C102 & $-0.0447(4)$ & $0.49543(17)$ & $-0.03114(18)$ & $0.0388(7)$ \\
\hline H102 & -0.1084 & 0.5361 & -0.0520 & $0.047 *$ \\
\hline C103 & $0.0063(3)$ & $0.44113(16)$ & $-0.08976(17)$ & $0.0345(6)$ \\
\hline C104 & 0.0958 & $0.38254(16)$ & $-0.05536(16)$ & $0.0340(6)$ \\
\hline $\mathrm{C} 105$ & $0.2807(4)$ & $0.29429(17)$ & $-0.03854(17)$ & $0.0381(7)$ \\
\hline $\mathrm{H} 105$ & 0.2992 & 0.2383 & -0.0495 & $0.046^{*}$ \\
\hline C106 & $0.4274(4)$ & $0.34060(18)$ & $-0.04789(18)$ & $0.0426(7)$ \\
\hline $\mathrm{C} 107$ & $0.5333(4)$ & $0.3442(2)$ & $0.02958(19)$ & $0.0467(8)$ \\
\hline H10B & 0.6185 & 0.3793 & 0.0182 & $0.056^{*}$ \\
\hline $\mathrm{H} 10 \mathrm{C}$ & 0.5753 & 0.2923 & 0.0421 & $0.056^{*}$ \\
\hline C108 & $0.4500(4)$ & $0.37357(18)$ & $0.10733(18)$ & $0.0404(7)$ \\
\hline H10D & 0.5220 & 0.3783 & 0.1577 & $0.049 *$ \\
\hline $\mathrm{H} 10 \mathrm{E}$ & 0.4067 & 0.4252 & 0.0946 & $0.049 *$ \\
\hline C109 & 0.2282 & $0.34699(16)$ & $0.20183(16)$ & $0.0334(6)$ \\
\hline H109 & 0.2988 & 0.3561 & 0.2529 & $0.040 *$ \\
\hline C110 & $0.1418(4)$ & $0.42301(17)$ & $0.18302(17)$ & $0.0389(7)$ \\
\hline H11A & 0.0552 & 0.4259 & 0.2209 & $0.047 *$ \\
\hline H11B & 0.2099 & 0.4669 & 0.1978 & $0.047 *$ \\
\hline C111 & $0.0831(3)$ & $0.43183(16)$ & $0.09051(17)$ & $0.0349(6)$ \\
\hline C112 & $0.1276(3)$ & $0.37784(16)$ & $0.03185(17)$ & $0.0318(6)$ \\
\hline C113 & 0.2135 & $0.30510(15)$ & $0.05051(16)$ & $0.0323(6)$ \\
\hline C114 & $0.3243(3)$ & $0.31769(16)$ & $0.12767(17)$ & $0.0332(6)$ \\
\hline C115 & $0.1007(3)$ & $0.24068(16)$ & $0.07105(17)$ & $0.0364(6)$ \\
\hline $\mathrm{H} 11 \mathrm{C}$ & 0.0276 & 0.2337 & 0.0215 & $0.044 *$ \\
\hline H11D & 0.1561 & 0.1915 & 0.0803 & $0.044 *$ \\
\hline $\mathrm{C} 116$ & $0.0155(3)$ & $0.26007(18)$ & $0.15079(17)$ & $0.0371(6)$ \\
\hline
\end{tabular}




\begin{tabular}{|c|c|c|c|c|}
\hline H11E & -0.0534 & 0.3041 & 0.1385 & $0.045^{*}$ \\
\hline $\mathrm{H} 11 \mathrm{~F}$ & -0.0464 & 0.2152 & 0.1670 & $0.045^{*}$ \\
\hline $\mathrm{C} 117$ & 0.0460 (3) & $0.28935(18)$ & $0.30721(17)$ & $0.0365(6)$ \\
\hline H11G & -0.0190 & 0.3358 & 0.3037 & $0.044^{*}$ \\
\hline $\mathrm{H} 11 \mathrm{H}$ & -0.0200 & 0.2441 & 0.3153 & $0.044^{*}$ \\
\hline C118 & 0.1548 (4) & $0.29650(17)$ & 0.38299 (17) & $0.0382(6)$ \\
\hline H118 & 0.2136 & 0.3457 & 0.3873 & $0.046^{*}$ \\
\hline C119 & $0.2336(4)$ & $0.22618(18)$ & 0.41855 (19) & $0.0466(8)$ \\
\hline H11I & 0.2121 & 0.1763 & 0.3895 & $0.056^{*}$ \\
\hline H11J & 0.3386 & 0.2322 & 0.4424 & $0.056^{*}$ \\
\hline $\mathrm{C} 120$ & $0.1099(5)$ & $0.2623(2)$ & 0.46651 (19) & $0.0528(9)$ \\
\hline $\mathrm{H} 12 \mathrm{~A}$ & 0.1385 & 0.2907 & 0.5200 & $0.063^{*}$ \\
\hline H12B & 0.0120 & 0.2347 & 0.4671 & $0.063^{*}$ \\
\hline O1 & $0.0294(3)$ & $0.47448(14)$ & $0.37422(13)$ & $0.0511(6)$ \\
\hline $\mathrm{O} 2$ & $0.3248(2)$ & $0.51172(11)$ & 0.40399 (11) & $0.0368(4)$ \\
\hline $\mathrm{O} 3$ & 0.5194 (3) & $0.39454(13)$ & $0.34376(12)$ & $0.0436(5)$ \\
\hline H3 & $0.570(5)$ & $0.355(2)$ & $0.3345(7)$ & $0.065^{*}$ \\
\hline O4 & $0.1098(2)$ & $0.60254(11)$ & $0.64191(12)$ & $0.0358(4)$ \\
\hline H4 & $0.036(4)$ & $0.5974(3)$ & $0.675(2)$ & $0.054^{*}$ \\
\hline N1 & 0.3739 (3) & $0.56903(14)$ & $0.72755(13)$ & $0.0324(5)$ \\
\hline H1 & 0.310 (4) & 0.6157 (19) & $0.731(2)$ & $0.039 *$ \\
\hline $\mathrm{C} 1$ & $0.5082(4)$ & $0.35396(17)$ & 0.57788 (19) & $0.0402(7)$ \\
\hline $\mathrm{H} 1 \mathrm{~A}$ & 0.5453 & 0.3171 & 0.6188 & $0.048^{*}$ \\
\hline $\mathrm{C} 2$ & 0.5475 (3) & $0.34791(18)$ & 0.49230 (19) & $0.0403(7)$ \\
\hline H2 & 0.6140 & 0.3078 & 0.4767 & $0.048^{*}$ \\
\hline $\mathrm{C} 3$ & $0.4921(3)$ & 0.39885 (17) & $0.42884(17)$ & $0.0355(6)$ \\
\hline $\mathrm{C} 4$ & $0.3974(3)$ & $0.45702(16)$ & 0.45557 (17) & $0.0326(6)$ \\
\hline $\mathrm{C} 5$ & $0.2118(3)$ & 0.54665 (16) & $0.45837(16)$ & $0.0345(6)$ \\
\hline H5 & 0.1983 & 0.6025 & 0.4435 & $0.041 *$ \\
\hline C6 & $0.0611(4)$ & $0.50333(16)$ & 0.44294 (18) & $0.0369(6)$ \\
\hline $\mathrm{C} 7$ & $-0.0406(4)$ & 0.49965 (19) & $0.51721(19)$ & $0.0422(7)$ \\
\hline H7A & -0.1257 & 0.4641 & 0.5030 & $0.051 *$ \\
\hline H7B & -0.0834 & 0.5515 & 0.5273 & $0.051 *$ \\
\hline $\mathrm{C} 8$ & $0.0453(3)$ & $0.47173(17)$ & $0.59927(18)$ & $0.0366(6)$ \\
\hline $\mathrm{H} 8 \mathrm{~A}$ & -0.0252 & 0.4675 & 0.6466 & $0.044 *$ \\
\hline H8B & 0.0889 & 0.4200 & 0.5894 & $0.044 *$ \\
\hline C9 & $0.2696(3)$ & $0.50184(16)$ & $0.70365(16)$ & $0.0331(6)$ \\
\hline H9 & 0.2003 & 0.4946 & 0.7519 & $0.040^{*}$ \\
\hline $\mathrm{C} 10$ & $0.3548(4)$ & $0.42536(17)$ & $0.69254(17)$ & $0.0381(7)$ \\
\hline $\mathrm{H} 10 \mathrm{~F}$ & 0.4407 & 0.4233 & 0.7355 & $0.046^{*}$ \\
\hline H10G & 0.2859 & 0.3821 & 0.7048 & $0.046^{*}$ \\
\hline C11 & 0.4147 (3) & 0.41385 (16) & $0.60374(17)$ & 0.0349 (6) \\
\hline $\mathrm{C} 12$ & $0.3663(3)$ & $0.46504(15)$ & $0.54039(16)$ & $0.0310(6)$ \\
\hline $\mathrm{C} 13$ & $0.2796(3)$ & $0.53846(15)$ & $0.55069(16)$ & $0.0310(6)$ \\
\hline $\mathrm{C} 14$ & $0.1716(3)$ & $0.52858(15)$ & 0.62424 (16) & $0.0314(6)$ \\
\hline $\mathrm{C} 15$ & 0.3919 (3) & 0.60404 (16) & $0.57368(16)$ & $0.0343(6)$ \\
\hline $\mathrm{H} 15 \mathrm{~A}$ & 0.4629 & 0.6101 & 0.5269 & $0.041^{*}$ \\
\hline H15B & 0.3357 & 0.6531 & 0.5790 & $0.041^{*}$ \\
\hline
\end{tabular}




\begin{tabular}{|c|c|c|c|c|}
\hline C16 & 0.4808 (3) & $0.58751(18)$ & $0.65763(16)$ & $0.0360(6)$ \\
\hline H16A & 0.5498 & 0.5434 & 0.6498 & $0.043^{*}$ \\
\hline H16B & 0.5429 & 0.6331 & 0.6745 & $0.043^{*}$ \\
\hline $\mathrm{C} 17$ & $0.4593(3)$ & $0.56109(18)$ & $0.81328(17)$ & $0.0376(6)$ \\
\hline H17A & 0.5250 & 0.5149 & 0.8120 & $0.045^{*}$ \\
\hline H17B & 0.5252 & 0.6067 & 0.8228 & $0.045^{*}$ \\
\hline $\mathrm{C} 18$ & 0.3563 (4) & $0.55394(17)$ & $0.88672(17)$ & 0.0389 (7) \\
\hline H18 & 0.2991 & 0.5044 & 0.8905 & $0.047^{*}$ \\
\hline C19 & 0.4090 (4) & 0.5899 (2) & $0.97063(18)$ & $0.0524(8)$ \\
\hline H19A & 0.3863 & 0.5624 & 1.0242 & $0.063^{*}$ \\
\hline H19B & 0.5066 & 0.6180 & 0.9727 & $0.063 *$ \\
\hline $\mathrm{C} 20$ & $0.2796(4)$ & $0.62445(19)$ & $0.9190(2)$ & $0.0472(8)$ \\
\hline $\mathrm{H} 20 \mathrm{~A}$ & 0.2972 & 0.6739 & 0.8892 & $0.057^{*}$ \\
\hline $\mathrm{H} 20 \mathrm{~B}$ & 0.1769 & 0.6183 & 0.9407 & $0.057 *$ \\
\hline O5 & $0.4669(3)$ & $0.57652(18)$ & $0.25608(17)$ & $0.0659(8)$ \\
\hline H5A & $0.4432(18)$ & $0.545(3)$ & $0.297(3)$ & $0.099 *$ \\
\hline $\mathrm{C} 21$ & $0.6265(4)$ & 0.5937 (3) & $0.2645(2)$ & $0.0548(9)$ \\
\hline $\mathrm{C} 22$ & 0.6449 (5) & $0.6660(3)$ & 0.2117 (3) & $0.0857(16)$ \\
\hline $\mathrm{H} 22 \mathrm{~A}$ & 0.5998 & 0.6579 & 0.1537 & $0.129^{*}$ \\
\hline H22B & 0.7527 & 0.6778 & 0.2082 & $0.129^{*}$ \\
\hline $\mathrm{H} 22 \mathrm{C}$ & 0.5940 & 0.7091 & 0.2390 & $0.129 *$ \\
\hline $\mathrm{C} 23$ & $0.7149(5)$ & $0.5272(3)$ & $0.2279(3)$ & $0.0768(13)$ \\
\hline $\mathrm{H} 23 \mathrm{~A}$ & 0.6925 & 0.4797 & 0.2589 & $0.115^{*}$ \\
\hline $\mathrm{H} 23 \mathrm{~B}$ & 0.8234 & 0.5382 & 0.2344 & $0.115^{*}$ \\
\hline $\mathrm{H} 23 \mathrm{C}$ & 0.6858 & 0.5209 & 0.1668 & $0.115^{*}$ \\
\hline $\mathrm{C} 24$ & $0.6731(4)$ & $0.6062(3)$ & $0.3577(2)$ & $0.0644(10)$ \\
\hline $\mathrm{H} 24 \mathrm{~A}$ & 0.6150 & 0.6491 & 0.3805 & $0.097 *$ \\
\hline H24B & 0.7813 & 0.6184 & 0.3630 & $0.097^{*}$ \\
\hline $\mathrm{H} 24 \mathrm{C}$ & 0.6534 & 0.5592 & 0.3904 & $0.097 *$ \\
\hline O6 & $0.2828(3)$ & $0.69659(15)$ & $0.32281(17)$ & $0.0634(7)$ \\
\hline H6 & 0.3391 & 0.6639 & 0.3005 & $0.095^{*}$ \\
\hline C31 & 0.1740 (4) & $0.7253(2)$ & $0.2583(2)$ & $0.0490(8)$ \\
\hline C32 & $0.0606(6)$ & $0.7709(3)$ & $0.3054(3)$ & $0.0751(12)$ \\
\hline $\mathrm{H} 32 \mathrm{~A}$ & 0.0103 & 0.7371 & 0.3457 & $0.113 *$ \\
\hline H32B & -0.0149 & 0.7927 & 0.2642 & $0.113^{*}$ \\
\hline $\mathrm{H} 32 \mathrm{C}$ & 0.1121 & 0.8128 & 0.3373 & $0.113^{*}$ \\
\hline C33 & $0.2538(5)$ & $0.7753(3)$ & 0.1944 (3) & $0.0766(13)$ \\
\hline $\mathrm{H} 33 \mathrm{~A}$ & 0.2990 & 0.8201 & 0.2242 & $0.115^{*}$ \\
\hline H33B & 0.1806 & 0.7929 & 0.1494 & $0.115^{*}$ \\
\hline $\mathrm{H} 33 \mathrm{C}$ & 0.3334 & 0.7452 & 0.1683 & $0.115^{*}$ \\
\hline C34 & $0.1000(5)$ & 0.6567 (3) & $0.2120(3)$ & $0.0744(12)$ \\
\hline $\mathrm{H} 34 \mathrm{~A}$ & 0.1780 & 0.6253 & 0.1861 & $0.112 *$ \\
\hline H34B & 0.0281 & 0.6752 & 0.1668 & $0.112^{*}$ \\
\hline $\mathrm{H} 34 \mathrm{C}$ & 0.0464 & 0.6253 & 0.2532 & $0.112 *$ \\
\hline
\end{tabular}


Atomic displacement parameters $\left(\AA^{2}\right)$

\begin{tabular}{|c|c|c|c|c|c|c|}
\hline & $U^{11}$ & $U^{22}$ & $U^{33}$ & $U^{12}$ & $U^{13}$ & $U^{23}$ \\
\hline $\mathrm{Cl1}$ & $0.0394(4)$ & $0.0395(4)$ & $0.0390(4)$ & 0.0071 & $-0.0031(3)$ & $-0.0032(3)$ \\
\hline $\mathrm{Cl} 2$ & 0.0407 (4) & $0.0366(3)$ & $0.0348(3)$ & $0.0083(3)$ & 0.0064 & 0.0045 \\
\hline O101 & $0.0668(16)$ & $0.0700(16)$ & $0.0344(12)$ & $0.0128(13)$ & $0.0152(11)$ & $0.0156(10)$ \\
\hline O102 & $0.0554(13)$ & $0.0410(11)$ & $0.0228(9)$ & $0.0142(10)$ & $-0.0012(9)$ & $-0.0015(7)$ \\
\hline O103 & $0.0544(14)$ & $0.0419(11)$ & $0.0258(9)$ & $0.0102(10)$ & $-0.0034(9)$ & $0.0043(8)$ \\
\hline O104 & $0.0388(11)$ & $0.0355(10)$ & $0.0340(10)$ & $0.0020(9)$ & $-0.0028(8)$ & $0.0083(8)$ \\
\hline N101 & $0.0380(13)$ & $0.0331(12)$ & $0.0260(11)$ & $-0.0001(10)$ & $-0.0022(10)$ & $0.0003(8)$ \\
\hline C101 & 0.0500 (19) & $0.0358(15)$ & $0.0323(15)$ & $0.0072(13)$ & $0.0061(13)$ & $-0.0032(11)$ \\
\hline C102 & $0.0448(17)$ & $0.0379(15)$ & $0.0338(15)$ & $0.0090(13)$ & $0.0024(13)$ & $0.0058(11)$ \\
\hline C103 & $0.0398(16)$ & $0.0374(15)$ & $0.0258(13)$ & $0.0011(12)$ & $-0.0022(11)$ & 0.0057 (11) \\
\hline C104 & $0.0417(16)$ & $0.0355(14)$ & $0.0248(13)$ & $0.0023(12)$ & $0.0023(11)$ & $-0.0014(10)$ \\
\hline $\mathrm{C} 105$ & $0.0515(18)$ & $0.0364(15)$ & $0.0264(13)$ & $0.0147(14)$ & $0.0023(12)$ & $-0.0005(11)$ \\
\hline C106 & $0.054(2)$ & $0.0401(16)$ & $0.0348(16)$ & $0.0176(15)$ & $0.0113(13)$ & $0.0051(12)$ \\
\hline $\mathrm{C} 107$ & $0.0446(18)$ & $0.055(2)$ & $0.0412(17)$ & $0.0027(16)$ & $0.0067(14)$ & $0.0161(14)$ \\
\hline $\mathrm{C} 108$ & 0.0469 (18) & $0.0418(16)$ & $0.0322(15)$ & $-0.0051(14)$ & $-0.0023(13)$ & $0.0093(12)$ \\
\hline C109 & $0.0437(16)$ & $0.0331(14)$ & $0.0230(12)$ & $-0.0057(12)$ & $-0.0020(11)$ & $0.0012(10)$ \\
\hline $\mathrm{C} 110$ & 0.0562 (19) & $0.0344(15)$ & $0.0262(13)$ & $0.0010(14)$ & $0.0019(13)$ & $-0.0030(11)$ \\
\hline C111 & $0.0450(17)$ & $0.0334(14)$ & $0.0264(13)$ & $0.0020(12)$ & $0.0042(11)$ & $0.0009(10)$ \\
\hline C112 & $0.0381(15)$ & $0.0315(14)$ & $0.0256(13)$ & $0.0015(12)$ & $-0.0014(11)$ & $0.0012(10)$ \\
\hline C113 & $0.0423(16)$ & $0.0328(14)$ & $0.0217(12)$ & $0.0027(12)$ & $-0.0001(11)$ & $0.0003(10)$ \\
\hline C114 & $0.0402(15)$ & $0.0325(14)$ & $0.0266(13)$ & $0.0022(12)$ & $-0.0017(11)$ & $0.0049(10)$ \\
\hline C115 & 0.0477 (17) & $0.0328(14)$ & $0.0279(13)$ & $-0.0011(13)$ & $-0.0067(12)$ & $-0.0015(10)$ \\
\hline C116 & 0.0393 (16) & $0.0397(15)$ & $0.0317(14)$ & $-0.0052(13)$ & $-0.0054(12)$ & $-0.0015(12)$ \\
\hline C117 & 0.0394 (16) & $0.0413(15)$ & $0.0290(13)$ & $-0.0001(13)$ & $0.0039(12)$ & $0.0002(11)$ \\
\hline C118 & $0.0473(18)$ & $0.0406(16)$ & $0.0269(13)$ & $-0.0029(14)$ & $0.0031(12)$ & $-0.0034(11)$ \\
\hline C119 & $0.060(2)$ & $0.0448(17)$ & $0.0339(15)$ & $0.0020(15)$ & $-0.0078(14)$ & $-0.0048(12)$ \\
\hline C120 & $0.076(2)$ & $0.056(2)$ & $0.0265(14)$ & -0.0048 (19) & $0.0075(15)$ & $-0.0012(13)$ \\
\hline $\mathrm{O} 1$ & $0.0558(14)$ & $0.0648(15)$ & $0.0321(11)$ & 0.0027 (12) & $-0.0044(10)$ & $-0.0109(10)$ \\
\hline $\mathrm{O} 2$ & $0.0459(12)$ & 0.0403 (11) & $0.0246(9)$ & $0.0059(9)$ & $0.0072(8)$ & $0.0036(7)$ \\
\hline $\mathrm{O} 3$ & 0.0497 (14) & 0.0495 (13) & $0.0322(10)$ & $0.0098(10)$ & $0.0088(9)$ & $-0.0066(8)$ \\
\hline $\mathrm{O} 4$ & $0.0384(11)$ & $0.0344(10)$ & $0.0351(10)$ & $0.0009(9)$ & $0.0069(8)$ & $-0.0064(8)$ \\
\hline N1 & 0.0375 & $0.0353(12)$ & $0.0247(11)$ & $-0.0029(10)$ & $0.0035(10)$ & $-0.0029(9)$ \\
\hline $\mathrm{C} 1$ & $0.0474(18)$ & $0.0362(15)$ & $0.0364(15)$ & 0.0039 (14) & $-0.0036(13)$ & $0.0008(12)$ \\
\hline $\mathrm{C} 2$ & $0.0425(17)$ & $0.0375(16)$ & $0.0407(16)$ & $0.0060(14)$ & $0.0001(13)$ & $-0.0056(12)$ \\
\hline $\mathrm{C} 3$ & $0.0365(15)$ & $0.0402(15)$ & $0.0299(14)$ & $-0.0018(13)$ & $0.0037(12)$ & $-0.0067(11)$ \\
\hline $\mathrm{C} 4$ & $0.0359(15)$ & $0.0328(14)$ & $0.0292(13)$ & $-0.0015(12)$ & $0.0027(11)$ & $0.0015(10)$ \\
\hline $\mathrm{C} 5$ & $0.0442(16)$ & $0.0345(14)$ & $0.0251(13)$ & $0.0067(12)$ & $0.0051(11)$ & $0.0014(10)$ \\
\hline C6 & 0.0463 (17) & $0.0342(14)$ & $0.0298(14)$ & $0.0073(13)$ & $-0.0034(12)$ & $-0.0025(11)$ \\
\hline $\mathrm{C} 7$ & $0.0406(17)$ & $0.0491(18)$ & $0.0368(16)$ & $-0.0016(14)$ & $0.0003(13)$ & $-0.0092(13)$ \\
\hline $\mathrm{C} 8$ & $0.0396(16)$ & $0.0412(16)$ & $0.0294(14)$ & $-0.0074(13)$ & $0.0052(12)$ & $-0.0048(11)$ \\
\hline $\mathrm{C} 9$ & $0.0398(16)$ & $0.0364(15)$ & $0.0236(12)$ & $-0.0063(12)$ & $0.0063(11)$ & $-0.0017(10)$ \\
\hline $\mathrm{C} 10$ & 0.0535 (19) & $0.0359(15)$ & $0.0248(13)$ & $-0.0039(14)$ & $0.0010(12)$ & 0.0035 (11) \\
\hline C11 & $0.0412(16)$ & $0.0341(14)$ & $0.0293(14)$ & $-0.0001(13)$ & $0.0012(12)$ & $0.0004(10)$ \\
\hline $\mathrm{C} 12$ & $0.0353(14)$ & $0.0322(13)$ & $0.0257(13)$ & 0.0005 (12) & $0.0031(11)$ & $-0.0018(10)$ \\
\hline C13 & $0.0386(15)$ & $0.0292(13)$ & $0.0253(13)$ & -0.0003 & $0.0034(11)$ & $0.0010(10)$ \\
\hline
\end{tabular}




\begin{tabular}{lllllll} 
C14 & $0.0369(15)$ & $0.0311(14)$ & $0.0265(12)$ & $-0.0017(12)$ & $0.0050(11)$ & $-0.0020(10)$ \\
C15 & $0.0410(15)$ & $0.0342(14)$ & $0.0284(12)$ & $-0.0055(13)$ & $0.0088(11)$ & $0.0002(11)$ \\
C16 & $0.0378(15)$ & $0.0404(15)$ & $0.0303(13)$ & $-0.0067(14)$ & $0.0065(11)$ & $-0.0024(12)$ \\
C17 & $0.0417(17)$ & $0.0415(15)$ & $0.0292(14)$ & $0.0017(13)$ & $-0.0018(12)$ & $-0.0008(11)$ \\
C18 & $0.0467(17)$ & $0.0423(16)$ & $0.0273(14)$ & $-0.0024(14)$ & $-0.0011(12)$ & $0.0038(11)$ \\
C19 & $0.070(2)$ & $0.059(2)$ & $0.0279(14)$ & $-0.001(2)$ & $0.0000(14)$ & $0.0004(14)$ \\
C20 & $0.059(2)$ & $0.0463(17)$ & $0.0377(16)$ & $0.0036(16)$ & $0.0123(15)$ & $0.0032(13)$ \\
O5 & $0.0436(13)$ & $0.096(2)$ & $0.0582(15)$ & $-0.0102(14)$ & $0.0012(11)$ & $0.0354(14)$ \\
C21 & $0.0395(17)$ & $0.075(2)$ & $0.0502(18)$ & $-0.0067(19)$ & $0.0057(14)$ & $0.0212(18)$ \\
C22 & $0.057(3)$ & $0.104(4)$ & $0.096(3)$ & $-0.011(3)$ & $0.003(2)$ & $0.053(3)$ \\
C23 & $0.064(3)$ & $0.093(3)$ & $0.075(3)$ & $-0.017(3)$ & $0.019(2)$ & $-0.006(2)$ \\
C24 & $0.056(2)$ & $0.082(3)$ & $0.055(2)$ & $-0.008(2)$ & $0.0016(17)$ & $0.0049(19)$ \\
O6 & $0.0667(18)$ & $0.0617(17)$ & $0.0604(16)$ & $-0.0029(13)$ & $-0.0104(13)$ & $0.0094(12)$ \\
C31 & $0.0449(19)$ & $0.0504(19)$ & $0.0513(19)$ & $-0.0031(15)$ & $-0.0002(15)$ & $0.0067(14)$ \\
C32 & $0.086(3)$ & $0.073(3)$ & $0.067(3)$ & $0.013(3)$ & $0.013(2)$ & $-0.002(2)$ \\
C33 & $0.062(3)$ & $0.085(3)$ & $0.082(3)$ & $-0.002(2)$ & $0.005(2)$ & $0.034(2)$ \\
C34 & $0.058(3)$ & $0.072(3)$ & $0.092(3)$ & $0.000(2)$ & $-0.007(2)$ & $-0.020(2)$ \\
& & & & & & \\
\hline
\end{tabular}

Geometric parameters $\left(A,{ }^{\circ}\right)$

\begin{tabular}{llll}
\hline $\mathrm{O} 101-\mathrm{C} 106$ & $1.203(4)$ & $\mathrm{C} 2-\mathrm{H} 2$ & 0.9500 \\
$\mathrm{O} 102-\mathrm{C} 104$ & $1.387(3)$ & $\mathrm{C} 2-\mathrm{C} 3$ & $1.396(4)$ \\
$\mathrm{O} 102-\mathrm{C} 105$ & $1.459(3)$ & $\mathrm{C} 3-\mathrm{C} 4$ & $1.387(4)$ \\
$\mathrm{O} 103-\mathrm{H} 103$ & $0.87(4)$ & $\mathrm{C} 4-\mathrm{C} 12$ & $1.369(4)$ \\
$\mathrm{O} 103-\mathrm{C} 103$ & $1.358(3)$ & $\mathrm{C} 5-\mathrm{H} 5$ & 1.0000 \\
$\mathrm{O} 104-\mathrm{H} 104$ & $0.87(4)$ & $\mathrm{C} 5-\mathrm{C} 6$ & $1.539(4)$ \\
$\mathrm{O} 104-\mathrm{C} 114$ & $1.425(3)$ & $\mathrm{C} 5-\mathrm{C} 13$ & $1.538(4)$ \\
$\mathrm{N} 101-\mathrm{H} 101$ & $0.84(3)$ & $\mathrm{C} 6-\mathrm{C} 7$ & $1.500(4)$ \\
$\mathrm{N} 101-\mathrm{C} 109$ & $1.520(4)$ & $\mathrm{C} 7-\mathrm{H} 7 \mathrm{~A}$ & 0.9900 \\
$\mathrm{~N} 101-\mathrm{C} 116$ & $1.500(3)$ & $\mathrm{C} 7-\mathrm{H} 7 \mathrm{~B}$ & 0.9900 \\
$\mathrm{~N} 101-\mathrm{C} 117$ & $1.513(3)$ & $\mathrm{C} 7-\mathrm{C} 8$ & $1.534(4)$ \\
$\mathrm{C} 101-\mathrm{H} 10 \mathrm{~A}$ & 0.9500 & $\mathrm{C} 8-\mathrm{H} 8 \mathrm{~A}$ & 0.9900 \\
$\mathrm{C} 101-\mathrm{C} 102$ & $1.388(4)$ & $\mathrm{C} 8-\mathrm{H} 8 \mathrm{~B}$ & 0.9900 \\
$\mathrm{C} 101-\mathrm{C} 111$ & $1.399(4)$ & $\mathrm{C} 8-\mathrm{C} 14$ & $1.526(4)$ \\
$\mathrm{C} 102-\mathrm{H} 102$ & 0.9500 & $\mathrm{C} 9-\mathrm{H} 9$ & 1.0000 \\
$\mathrm{C} 102-\mathrm{C} 103$ & $1.400(4)$ & $\mathrm{C} 9-\mathrm{C} 10$ & $1.538(4)$ \\
$\mathrm{C} 103-\mathrm{C} 104$ & $1.380(4)$ & $\mathrm{C} 9-\mathrm{C} 14$ & $1.548(4)$ \\
$\mathrm{C} 104-\mathrm{C} 112$ & $1.376(4)$ & $\mathrm{C} 10-\mathrm{H} 10 \mathrm{~F}$ & 0.9900 \\
$\mathrm{C} 105-\mathrm{H} 105$ & 1.0000 & $\mathrm{C} 10-\mathrm{H} 10 \mathrm{G}$ & 0.9900 \\
$\mathrm{C} 105-\mathrm{C} 106$ & $1.539(5)$ & $\mathrm{C} 10-\mathrm{C} 11$ & $1.517(4)$ \\
$\mathrm{C} 105-\mathrm{C} 113$ & $1.545(4)$ & $\mathrm{C} 11-\mathrm{C} 12$ & $1.380(4)$ \\
$\mathrm{C} 106-\mathrm{C} 107$ & $1.494(4)$ & $\mathrm{C} 12-\mathrm{C} 13$ & $1.498(4)$ \\
$\mathrm{C} 107-\mathrm{H} 10 \mathrm{~B}$ & 0.9900 & $\mathrm{C} 13-\mathrm{C} 14$ & $1.535(4)$ \\
$\mathrm{C} 107-\mathrm{H} 10 \mathrm{C}$ & 0.9900 & $\mathrm{C} 13-\mathrm{C} 15$ & $1.541(4)$ \\
$\mathrm{C} 107-\mathrm{C} 108$ & $1.533(4)$ & $\mathrm{C} 15-\mathrm{H} 15 \mathrm{~A}$ & 0.9900 \\
$\mathrm{C} 108-\mathrm{H} 10 \mathrm{D}$ & 0.9900 & $\mathrm{C} 15-\mathrm{H} 15 \mathrm{~B}$ & 0.9900 \\
$\mathrm{C} 108-\mathrm{H} 10 \mathrm{E}$ & 0.9900 & $\mathrm{C} 15-\mathrm{C} 16$ & $1.521(4)$ \\
$\mathrm{C} 108-\mathrm{C} 114$ & $1.520(4)$ & $\mathrm{C} 16-\mathrm{H} 16 \mathrm{~A}$ & 0.9900
\end{tabular}




\begin{tabular}{|c|c|c|c|}
\hline C109-H109 & 1.0000 & $\mathrm{C} 16-\mathrm{H} 16 \mathrm{~B}$ & 0.9900 \\
\hline $\mathrm{C} 109-\mathrm{C} 110$ & $1.544(4)$ & C17-H17A & 0.9900 \\
\hline $\mathrm{C} 109-\mathrm{C} 114$ & $1.551(4)$ & $\mathrm{C} 17-\mathrm{H} 17 \mathrm{~B}$ & 0.9900 \\
\hline C110-H11A & 0.9900 & $\mathrm{C} 17-\mathrm{C} 18$ & $1.500(4)$ \\
\hline $\mathrm{C} 110-\mathrm{H} 11 \mathrm{~B}$ & 0.9900 & $\mathrm{C} 18-\mathrm{H} 18$ & 1.0000 \\
\hline $\mathrm{C} 110-\mathrm{C} 111$ & $1.516(4)$ & $\mathrm{C} 18-\mathrm{C} 19$ & $1.502(4)$ \\
\hline $\mathrm{C} 111-\mathrm{C} 112$ & $1.378(4)$ & $\mathrm{C} 18-\mathrm{C} 20$ & $1.496(4)$ \\
\hline $\mathrm{C} 112-\mathrm{C} 113$ & $1.493(4)$ & C19-H19A & 0.9900 \\
\hline $\mathrm{C} 113-\mathrm{C} 114$ & $1.530(4)$ & С19-H19B & 0.9900 \\
\hline C113-C115 & $1.541(4)$ & $\mathrm{C} 19-\mathrm{C} 20$ & $1.493(5)$ \\
\hline $\mathrm{C} 115-\mathrm{H} 11 \mathrm{C}$ & 0.9900 & $\mathrm{C} 20-\mathrm{H} 20 \mathrm{~A}$ & 0.9900 \\
\hline C115-H11D & 0.9900 & $\mathrm{C} 20-\mathrm{H} 20 \mathrm{~B}$ & 0.9900 \\
\hline $\mathrm{C} 115-\mathrm{C} 116$ & $1.520(4)$ & $\mathrm{O} 5-\mathrm{H} 5 \mathrm{~A}$ & $0.87(5)$ \\
\hline C116-H11E & 0.9900 & $\mathrm{O} 5-\mathrm{C} 21$ & $1.443(4)$ \\
\hline $\mathrm{C} 116-\mathrm{H} 11 \mathrm{~F}$ & 0.9900 & $\mathrm{C} 21-\mathrm{C} 22$ & $1.512(6)$ \\
\hline $\mathrm{C} 117-\mathrm{H} 11 \mathrm{G}$ & 0.9900 & $\mathrm{C} 21-\mathrm{C} 23$ & $1.518(6)$ \\
\hline $\mathrm{C} 117-\mathrm{H} 11 \mathrm{H}$ & 0.9900 & $\mathrm{C} 21-\mathrm{C} 24$ & $1.506(5)$ \\
\hline C117-C118 & $1.492(4)$ & $\mathrm{C} 22-\mathrm{H} 22 \mathrm{~A}$ & 0.9800 \\
\hline C118-H118 & 1.0000 & $\mathrm{C} 22-\mathrm{H} 22 \mathrm{~B}$ & 0.9800 \\
\hline C118-C119 & $1.497(4)$ & $\mathrm{C} 22-\mathrm{H} 22 \mathrm{C}$ & 0.9800 \\
\hline $\mathrm{C} 118-\mathrm{C} 120$ & $1.500(4)$ & $\mathrm{C} 23-\mathrm{H} 23 \mathrm{~A}$ & 0.9800 \\
\hline C119-H11I & 0.9900 & $\mathrm{C} 23-\mathrm{H} 23 \mathrm{~B}$ & 0.9800 \\
\hline $\mathrm{C} 119-\mathrm{H} 11 \mathrm{~J}$ & 0.9900 & $\mathrm{C} 23-\mathrm{H} 23 \mathrm{C}$ & 0.9800 \\
\hline $\mathrm{C} 119-\mathrm{C} 120$ & $1.491(5)$ & $\mathrm{C} 24-\mathrm{H} 24 \mathrm{~A}$ & 0.9800 \\
\hline $\mathrm{C} 120-\mathrm{H} 12 \mathrm{~A}$ & 0.9900 & $\mathrm{C} 24-\mathrm{H} 24 \mathrm{~B}$ & 0.9800 \\
\hline $\mathrm{C} 120-\mathrm{H} 12 \mathrm{~B}$ & 0.9900 & $\mathrm{C} 24-\mathrm{H} 24 \mathrm{C}$ & 0.9800 \\
\hline $\mathrm{O} 1-\mathrm{C} 6$ & $1.202(3)$ & O6-H6 & 0.8400 \\
\hline $\mathrm{O} 2-\mathrm{C} 4$ & $1.381(3)$ & $\mathrm{O} 6-\mathrm{C} 31$ & $1.447(4)$ \\
\hline $\mathrm{O} 2-\mathrm{C} 5$ & $1.471(3)$ & $\mathrm{C} 31-\mathrm{C} 32$ & $1.497(5)$ \\
\hline $\mathrm{O} 3-\mathrm{H} 3$ & $0.84(4)$ & $\mathrm{C} 31-\mathrm{C} 33$ & $1.519(5)$ \\
\hline $\mathrm{O} 3-\mathrm{C} 3$ & $1.360(3)$ & $\mathrm{C} 31-\mathrm{C} 34$ & $1.522(5)$ \\
\hline $\mathrm{O} 4-\mathrm{H} 4$ & $0.86(4)$ & $\mathrm{C} 32-\mathrm{H} 32 \mathrm{~A}$ & 0.9800 \\
\hline $\mathrm{O} 4-\mathrm{C} 14$ & $1.425(3)$ & $\mathrm{C} 32-\mathrm{H} 32 \mathrm{~B}$ & 0.9800 \\
\hline N1-H1 & $0.99(3)$ & $\mathrm{C} 32-\mathrm{H} 32 \mathrm{C}$ & 0.9800 \\
\hline $\mathrm{N} 1-\mathrm{C} 9$ & $1.521(4)$ & $\mathrm{C} 33-\mathrm{H} 33 \mathrm{~A}$ & 0.9800 \\
\hline $\mathrm{N} 1-\mathrm{C} 16$ & $1.510(3)$ & $\mathrm{C} 33-\mathrm{H} 33 \mathrm{~B}$ & 0.9800 \\
\hline $\mathrm{N} 1-\mathrm{C} 17$ & $1.509(3)$ & $\mathrm{C} 33-\mathrm{H} 33 \mathrm{C}$ & 0.9800 \\
\hline $\mathrm{C} 1-\mathrm{H} 1 \mathrm{~A}$ & 0.9500 & $\mathrm{C} 34-\mathrm{H} 34 \mathrm{~A}$ & 0.9800 \\
\hline $\mathrm{C} 1-\mathrm{C} 2$ & $1.397(4)$ & $\mathrm{C} 34-\mathrm{H} 34 \mathrm{~B}$ & 0.9800 \\
\hline $\mathrm{C} 1-\mathrm{C} 11$ & $1.398(4)$ & $\mathrm{C} 34-\mathrm{H} 34 \mathrm{C}$ & 0.9800 \\
\hline $\mathrm{C} 104-\mathrm{O} 102-\mathrm{C} 105$ & $104.2(2)$ & $\mathrm{C} 13-\mathrm{C} 5-\mathrm{H} 5$ & 110.0 \\
\hline $\mathrm{C} 103-\mathrm{O} 103-\mathrm{H} 103$ & 109.5 & $\mathrm{C} 13-\mathrm{C} 5-\mathrm{C} 6$ & $113.4(2)$ \\
\hline $\mathrm{C} 114-\mathrm{O} 104-\mathrm{H} 104$ & 109.5 & $\mathrm{O} 1-\mathrm{C} 6-\mathrm{C} 5$ & $120.4(3)$ \\
\hline $\mathrm{C} 109-\mathrm{N} 101-\mathrm{H} 101$ & 105.7 & $\mathrm{O} 1-\mathrm{C} 6-\mathrm{C} 7$ & $123.0(3)$ \\
\hline C116-N101-H101 & 105.7 & $\mathrm{C} 7-\mathrm{C} 6-\mathrm{C} 5$ & $116.6(2)$ \\
\hline C116-N101-C109 & $112.4(2)$ & $\mathrm{C} 6-\mathrm{C} 7-\mathrm{H} 7 \mathrm{~A}$ & 109.4 \\
\hline $\mathrm{C} 116-\mathrm{N} 101-\mathrm{C} 117$ & $111.4(2)$ & $\mathrm{C} 6-\mathrm{C} 7-\mathrm{H} 7 \mathrm{~B}$ & 109.4 \\
\hline
\end{tabular}


C117-N101-H101

C117-N101-C109

C102-C101-H10A

C102-C101-C111

C111-C101-H10A

$\mathrm{C} 101-\mathrm{C} 102-\mathrm{H} 102$

$\mathrm{C} 101-\mathrm{C} 102-\mathrm{C} 103$

$\mathrm{C} 103-\mathrm{C} 102-\mathrm{H} 102$

$\mathrm{O} 103-\mathrm{C} 103-\mathrm{C} 102$

$\mathrm{O} 103-\mathrm{C} 103-\mathrm{C} 104$

$\mathrm{C} 104-\mathrm{C} 103-\mathrm{C} 102$

C103-C104-O102

C112-C104-O102

$\mathrm{C} 112-\mathrm{C} 104-\mathrm{C} 103$

$\mathrm{O} 102-\mathrm{C} 105-\mathrm{H} 105$

$\mathrm{O} 102-\mathrm{C} 105-\mathrm{C} 106$

$\mathrm{O} 102-\mathrm{C} 105-\mathrm{C} 113$

$\mathrm{C} 106-\mathrm{C} 105-\mathrm{H} 105$

$\mathrm{C} 106-\mathrm{C} 105-\mathrm{C} 113$

C113-C105-H105

$\mathrm{O} 101-\mathrm{C} 106-\mathrm{C} 105$

$\mathrm{O} 101-\mathrm{C} 106-\mathrm{C} 107$

C107-C106-C105

C106-C107-H10B

C106-C107-H10C

$\mathrm{C} 106-\mathrm{C} 107-\mathrm{C} 108$

$\mathrm{H} 10 \mathrm{~B}-\mathrm{C} 107-\mathrm{H} 10 \mathrm{C}$

C108-C107-H10B

C108- C107-H10C

C107-C108-H10D

C107-C108-H10E

$\mathrm{H} 10 \mathrm{D}-\mathrm{C} 108-\mathrm{H} 10 \mathrm{E}$

C114-C108-C107

C114-C108-H10D

C114-C108-H10E

N101-C109-H109

$\mathrm{N} 101-\mathrm{C} 109-\mathrm{C} 110$

N101-C109-C114

$\mathrm{C} 110-\mathrm{C} 109-\mathrm{H} 109$

$\mathrm{C} 110-\mathrm{C} 109-\mathrm{C} 114$

C114-C109-H109

$\mathrm{C} 109-\mathrm{C} 110-\mathrm{H} 11 \mathrm{~A}$

C109-C110-H11B

H11A-C110-H11B

C111-C110-C109

C111-C110-H11A

C111-C110-H11B

C101-C111-C110
105.7

114.9 (2)

119.6

120.9 (3)

119.6

118.7

$122.6(3)$

118.7

$124.6(2)$

$119.4(3)$

$116.0(2)$

$127.2(2)$

$111.9(2)$

$120.9(3)$

110.1

$109.1(2)$

104.7 (2)

110.1

112.5 (2)

110.1

120.5 (3)

$123.0(3)$

116.5 (2)

109.6

109.6

110.2 (3)

108.1

109.6

109.6

109.7

109.7

108.2

109.7 (3)

109.7

109.7

107.5

$113.2(2)$

$105.9(2)$

107.5

114.9 (2)

107.5

108.7

108.7

107.6

114.3 (2)

108.7

108.7

$126.8(2)$
$\mathrm{C} 6-\mathrm{C} 7-\mathrm{C} 8$

$\mathrm{H} 7 \mathrm{~A}-\mathrm{C} 7-\mathrm{H} 7 \mathrm{~B}$

$\mathrm{C} 8-\mathrm{C} 7-\mathrm{H} 7 \mathrm{~A}$

$\mathrm{C} 8-\mathrm{C} 7-\mathrm{H} 7 \mathrm{~B}$

$\mathrm{C} 7-\mathrm{C} 8-\mathrm{H} 8 \mathrm{~A}$

C7-C $8-\mathrm{H} 8 \mathrm{~B}$

$\mathrm{H} 8 \mathrm{~A}-\mathrm{C} 8-\mathrm{H} 8 \mathrm{~B}$

$\mathrm{C} 14-\mathrm{C} 8-\mathrm{C} 7$

C14-C8-H8A

C14-C $8-\mathrm{H} 8 \mathrm{~B}$

$\mathrm{N} 1-\mathrm{C} 9-\mathrm{H} 9$

$\mathrm{N} 1-\mathrm{C} 9-\mathrm{C} 10$

$\mathrm{N} 1-\mathrm{C} 9-\mathrm{C} 14$

$\mathrm{C} 10-\mathrm{C} 9-\mathrm{H} 9$

$\mathrm{C} 10-\mathrm{C} 9-\mathrm{C} 14$

C14-C9-H9

C9- $\mathrm{C} 10-\mathrm{H} 10 \mathrm{~F}$

C9- $\mathrm{C} 10-\mathrm{H} 10 \mathrm{G}$

$\mathrm{H} 10 \mathrm{~F}-\mathrm{C} 10-\mathrm{H} 10 \mathrm{G}$

$\mathrm{C} 11-\mathrm{C} 10-\mathrm{C} 9$

$\mathrm{C} 11-\mathrm{C} 10-\mathrm{H} 10 \mathrm{~F}$

C11-C10-H10G

$\mathrm{C} 1-\mathrm{C} 11-\mathrm{C} 10$

$\mathrm{C} 12-\mathrm{C} 11-\mathrm{C} 1$

$\mathrm{C} 12-\mathrm{C} 11-\mathrm{C} 10$

$\mathrm{C} 4-\mathrm{C} 12-\mathrm{C} 11$

$\mathrm{C} 4-\mathrm{C} 12-\mathrm{C} 13$

$\mathrm{C} 11-\mathrm{C} 12-\mathrm{C} 13$

$\mathrm{C} 5-\mathrm{C} 13-\mathrm{C} 15$

$\mathrm{C} 12-\mathrm{C} 13-\mathrm{C} 5$

$\mathrm{C} 12-\mathrm{C} 13-\mathrm{C} 14$

C12-C13-C15

$\mathrm{C} 14-\mathrm{C} 13-\mathrm{C} 5$

$\mathrm{C} 14-\mathrm{C} 13-\mathrm{C} 15$

$\mathrm{O} 4-\mathrm{C} 14-\mathrm{C} 8$

$\mathrm{O} 4-\mathrm{C} 14-\mathrm{C} 9$

$\mathrm{O} 4-\mathrm{C} 14-\mathrm{C} 13$

$\mathrm{C} 8-\mathrm{C} 14-\mathrm{C} 9$

$\mathrm{C} 8-\mathrm{C} 14-\mathrm{C} 13$

C13-C14-C9

C13-C15-H15A

C13-C15-H15B

H15A-C15-H15B

$\mathrm{C} 16-\mathrm{C} 15-\mathrm{C} 13$

C16-C15-H15A

C16-C15-H15B

N1-C16-C15

N1-C16-H16A
$111.3(2)$

108.0

109.4

109.4

109.7

109.7

108.2

109.7 (2)

109.7

109.7

107.3

113.1 (2)

106.1 (2)

107.3

115.3 (2)

107.3

108.7

108.7

107.6

114.2 (2)

108.7

108.7

126.7 (3)

116.1 (2)

$117.2(3)$

123.7 (3)

108.5 (2)

127.8 (2)

111.6 (2)

99.1 (2)

109.0 (2)

108.9 (2)

$118.6(2)$

109.1 (2)

110.3 (2)

108.7 (2)

107.5 (2)

112.7 (2)

110.9 (2)

106.5 (2)

109.4

109.4

108.0

111.3 (2)

109.4

109.4

110.1 (2)

109.6 


\begin{tabular}{|c|c|c|c|}
\hline $\mathrm{C} 112-\mathrm{C} 111-\mathrm{C} 101$ & $115.3(2)$ & $\mathrm{N} 1-\mathrm{C} 16-\mathrm{H} 16 \mathrm{~B}$ & 109.6 \\
\hline $\mathrm{C} 112-\mathrm{C} 111-\mathrm{C} 110$ & $117.7(3)$ & $\mathrm{C} 15-\mathrm{C} 16-\mathrm{H} 16 \mathrm{~A}$ & 109.6 \\
\hline $\mathrm{C} 104-\mathrm{C} 112-\mathrm{C} 111$ & $124.2(3)$ & $\mathrm{C} 15-\mathrm{C} 16-\mathrm{H} 16 \mathrm{~B}$ & 109.6 \\
\hline $\mathrm{C} 104-\mathrm{C} 112-\mathrm{C} 113$ & $108.7(2)$ & $\mathrm{H} 16 \mathrm{~A}-\mathrm{C} 16-\mathrm{H} 16 \mathrm{~B}$ & 108.2 \\
\hline $\mathrm{C} 111-\mathrm{C} 112-\mathrm{C} 113$ & $127.1(2)$ & $\mathrm{N} 1-\mathrm{C} 17-\mathrm{H} 17 \mathrm{~A}$ & 109.1 \\
\hline $\mathrm{C} 112-\mathrm{C} 113-\mathrm{C} 105$ & $98.1(2)$ & $\mathrm{N} 1-\mathrm{C} 17-\mathrm{H} 17 \mathrm{~B}$ & 109.1 \\
\hline $\mathrm{C} 112-\mathrm{C} 113-\mathrm{C} 114$ & $109.5(2)$ & $\mathrm{H} 17 \mathrm{~A}-\mathrm{C} 17-\mathrm{H} 17 \mathrm{~B}$ & 107.8 \\
\hline $\mathrm{C} 112-\mathrm{C} 113-\mathrm{C} 115$ & $108.9(2)$ & $\mathrm{C} 18-\mathrm{C} 17-\mathrm{N} 1$ & $112.6(2)$ \\
\hline $\mathrm{C} 114-\mathrm{C} 113-\mathrm{C} 105$ & $117.6(3)$ & $\mathrm{C} 18-\mathrm{C} 17-\mathrm{H} 17 \mathrm{~A}$ & 109.1 \\
\hline $\mathrm{C} 114-\mathrm{C} 113-\mathrm{C} 115$ & $109.7(2)$ & $\mathrm{C} 18-\mathrm{C} 17-\mathrm{H} 17 \mathrm{~B}$ & 109.1 \\
\hline $\mathrm{C} 115-\mathrm{C} 113-\mathrm{C} 105$ & $112.2(2)$ & $\mathrm{C} 17-\mathrm{C} 18-\mathrm{H} 18$ & 116.3 \\
\hline $\mathrm{O} 104-\mathrm{C} 114-\mathrm{C} 108$ & $110.7(2)$ & $\mathrm{C} 17-\mathrm{C} 18-\mathrm{C} 19$ & $117.0(3)$ \\
\hline $\mathrm{O} 104-\mathrm{C} 114-\mathrm{C} 109$ & $108.3(2)$ & $\mathrm{C} 19-\mathrm{C} 18-\mathrm{H} 18$ & 116.3 \\
\hline $\mathrm{O} 104-\mathrm{C} 114-\mathrm{C} 113$ & $107.1(2)$ & $\mathrm{C} 20-\mathrm{C} 18-\mathrm{C} 17$ & $119.3(3)$ \\
\hline $\mathrm{C} 108-\mathrm{C} 114-\mathrm{C} 109$ & $112.3(2)$ & $\mathrm{C} 20-\mathrm{C} 18-\mathrm{H} 18$ & 116.3 \\
\hline $\mathrm{C} 108-\mathrm{C} 114-\mathrm{C} 113$ & $112.1(2)$ & $\mathrm{C} 20-\mathrm{C} 18-\mathrm{C} 19$ & $59.7(2)$ \\
\hline $\mathrm{C} 113-\mathrm{C} 114-\mathrm{C} 109$ & $106.2(2)$ & $\mathrm{C} 18-\mathrm{C} 19-\mathrm{H} 19 \mathrm{~A}$ & 117.8 \\
\hline $\mathrm{C} 113-\mathrm{C} 115-\mathrm{H} 11 \mathrm{C}$ & 109.4 & $\mathrm{C} 18-\mathrm{C} 19-\mathrm{H} 19 \mathrm{~B}$ & 117.8 \\
\hline $\mathrm{C} 113-\mathrm{C} 115-\mathrm{H} 11 \mathrm{D}$ & 109.4 & $\mathrm{H} 19 \mathrm{~A}-\mathrm{C} 19-\mathrm{H} 19 \mathrm{~B}$ & 114.9 \\
\hline $\mathrm{H} 11 \mathrm{C}-\mathrm{C} 115-\mathrm{H} 11 \mathrm{D}$ & 108.0 & $\mathrm{C} 20-\mathrm{C} 19-\mathrm{C} 18$ & $59.9(2)$ \\
\hline $\mathrm{C} 116-\mathrm{C} 115-\mathrm{C} 113$ & $111.1(2)$ & $\mathrm{C} 20-\mathrm{C} 19-\mathrm{H} 19 \mathrm{~A}$ & 117.8 \\
\hline $\mathrm{C} 116-\mathrm{C} 115-\mathrm{H} 11 \mathrm{C}$ & 109.4 & $\mathrm{C} 20-\mathrm{C} 19-\mathrm{H} 19 \mathrm{~B}$ & 117.8 \\
\hline $\mathrm{C} 116-\mathrm{C} 115-\mathrm{H} 11 \mathrm{D}$ & 109.4 & $\mathrm{C} 18-\mathrm{C} 20-\mathrm{H} 20 \mathrm{~A}$ & 117.7 \\
\hline $\mathrm{N} 101-\mathrm{C} 116-\mathrm{C} 115$ & $110.0(2)$ & $\mathrm{C} 18-\mathrm{C} 20-\mathrm{H} 20 \mathrm{~B}$ & 117.7 \\
\hline $\mathrm{N} 101-\mathrm{C} 116-\mathrm{H} 11 \mathrm{E}$ & 109.7 & $\mathrm{C} 19-\mathrm{C} 20-\mathrm{C} 18$ & $60.3(2)$ \\
\hline $\mathrm{N} 101-\mathrm{C} 116-\mathrm{H} 11 \mathrm{~F}$ & 109.7 & $\mathrm{C} 19-\mathrm{C} 20-\mathrm{H} 20 \mathrm{~A}$ & 117.7 \\
\hline $\mathrm{C} 115-\mathrm{C} 116-\mathrm{H} 11 \mathrm{E}$ & 109.7 & $\mathrm{C} 19-\mathrm{C} 20-\mathrm{H} 20 \mathrm{~B}$ & 117.7 \\
\hline $\mathrm{C} 115-\mathrm{C} 116-\mathrm{H} 11 \mathrm{~F}$ & 109.7 & $\mathrm{H} 20 \mathrm{~A}-\mathrm{C} 20-\mathrm{H} 20 \mathrm{~B}$ & 114.9 \\
\hline $\mathrm{H} 11 \mathrm{E}-\mathrm{C} 116-\mathrm{H} 11 \mathrm{~F}$ & 108.2 & $\mathrm{C} 21-\mathrm{O} 5-\mathrm{H} 5 \mathrm{~A}$ & 109.5 \\
\hline $\mathrm{N} 101-\mathrm{C} 117-\mathrm{H} 11 \mathrm{G}$ & 109.1 & $\mathrm{O} 5-\mathrm{C} 21-\mathrm{C} 22$ & $104.5(3)$ \\
\hline $\mathrm{N} 101-\mathrm{C} 117-\mathrm{H} 11 \mathrm{H}$ & 109.1 & $\mathrm{O} 5-\mathrm{C} 21-\mathrm{C} 23$ & $109.2(4)$ \\
\hline $\mathrm{H} 11 \mathrm{G}-\mathrm{C} 117-\mathrm{H} 11 \mathrm{H}$ & 107.9 & $\mathrm{O} 5-\mathrm{C} 21-\mathrm{C} 24$ & $109.8(3)$ \\
\hline $\mathrm{C} 118-\mathrm{C} 117-\mathrm{N} 101$ & $112.4(2)$ & $\mathrm{C} 22-\mathrm{C} 21-\mathrm{C} 23$ & $110.7(3)$ \\
\hline $\mathrm{C} 118-\mathrm{C} 117-\mathrm{H} 11 \mathrm{G}$ & 109.1 & $\mathrm{C} 24-\mathrm{C} 21-\mathrm{C} 22$ & $112.0(4)$ \\
\hline $\mathrm{C} 118-\mathrm{C} 117-\mathrm{H} 11 \mathrm{H}$ & 109.1 & $\mathrm{C} 24-\mathrm{C} 21-\mathrm{C} 23$ & $110.4(3)$ \\
\hline $\mathrm{C} 117-\mathrm{C} 118-\mathrm{H} 118$ & 116.0 & $\mathrm{C} 21-\mathrm{C} 22-\mathrm{H} 22 \mathrm{~A}$ & 109.5 \\
\hline $\mathrm{C} 117-\mathrm{C} 118-\mathrm{C} 119$ & $119.8(3)$ & $\mathrm{C} 21-\mathrm{C} 22-\mathrm{H} 22 \mathrm{~B}$ & 109.5 \\
\hline $\mathrm{C} 117-\mathrm{C} 118-\mathrm{C} 120$ & $117.7(3)$ & $\mathrm{C} 21-\mathrm{C} 22-\mathrm{H} 22 \mathrm{C}$ & 109.5 \\
\hline $\mathrm{C} 119-\mathrm{C} 118-\mathrm{H} 118$ & 116.0 & $\mathrm{H} 22 \mathrm{~A}-\mathrm{C} 22-\mathrm{H} 22 \mathrm{~B}$ & 109.5 \\
\hline $\mathrm{C} 119-\mathrm{C} 118-\mathrm{C} 120$ & $59.7(2)$ & $\mathrm{H} 22 \mathrm{~A}-\mathrm{C} 22-\mathrm{H} 22 \mathrm{C}$ & 109.5 \\
\hline $\mathrm{C} 120-\mathrm{C} 118-\mathrm{H} 118$ & 116.0 & $\mathrm{H} 22 \mathrm{~B}-\mathrm{C} 22-\mathrm{H} 22 \mathrm{C}$ & 109.5 \\
\hline $\mathrm{C} 118-\mathrm{C} 119-\mathrm{H} 11 \mathrm{I}$ & 117.7 & $\mathrm{C} 21-\mathrm{C} 23-\mathrm{H} 23 \mathrm{~A}$ & 109.5 \\
\hline $\mathrm{C} 118-\mathrm{C} 119-\mathrm{H} 11 \mathrm{~J}$ & 117.7 & $\mathrm{C} 21-\mathrm{C} 23-\mathrm{H} 23 \mathrm{~B}$ & 109.5 \\
\hline $\mathrm{H} 11 \mathrm{I}-\mathrm{C} 119-\mathrm{H} 11 \mathrm{~J}$ & 114.9 & $\mathrm{C} 21-\mathrm{C} 23-\mathrm{H} 23 \mathrm{C}$ & 109.5 \\
\hline $\mathrm{C} 120-\mathrm{C} 119-\mathrm{C} 118$ & $60.3(2)$ & $\mathrm{H} 23 \mathrm{~A}-\mathrm{C} 23-\mathrm{H} 23 \mathrm{~B}$ & 109.5 \\
\hline $\mathrm{C} 120-\mathrm{C} 119-\mathrm{H} 11 \mathrm{I}$ & 117.7 & $\mathrm{H} 23 \mathrm{~A}-\mathrm{C} 23-\mathrm{H} 23 \mathrm{C}$ & 109.5 \\
\hline $\mathrm{C} 120-\mathrm{C} 119-\mathrm{H} 11 \mathrm{~J}$ & 117.7 & $\mathrm{H} 23 \mathrm{~B}-\mathrm{C} 23-\mathrm{H} 23 \mathrm{C}$ & 109.5 \\
\hline $\mathrm{C} 118-\mathrm{C} 120-\mathrm{H} 12 \mathrm{~A}$ & 117.8 & $\mathrm{C} 21-\mathrm{C} 24-\mathrm{H} 24 \mathrm{~A}$ & 109.5 \\
\hline
\end{tabular}




\begin{tabular}{|c|c|c|c|}
\hline $\mathrm{C} 118-\mathrm{C} 120-\mathrm{H} 12 \mathrm{~B}$ & 117.8 & $\mathrm{C} 21-\mathrm{C} 24-\mathrm{H} 24 \mathrm{~B}$ & 109.5 \\
\hline $\mathrm{C} 119-\mathrm{C} 120-\mathrm{C} 118$ & $60.1(2)$ & $\mathrm{C} 21-\mathrm{C} 24-\mathrm{H} 24 \mathrm{C}$ & 109.5 \\
\hline $\mathrm{C} 119-\mathrm{C} 120-\mathrm{H} 12 \mathrm{~A}$ & 117.8 & $\mathrm{H} 24 \mathrm{~A}-\mathrm{C} 24-\mathrm{H} 24 \mathrm{~B}$ & 109.5 \\
\hline $\mathrm{C} 119-\mathrm{C} 120-\mathrm{H} 12 \mathrm{~B}$ & 117.8 & $\mathrm{H} 24 \mathrm{~A}-\mathrm{C} 24-\mathrm{H} 24 \mathrm{C}$ & 109.5 \\
\hline $\mathrm{H} 12 \mathrm{~A}-\mathrm{C} 120-\mathrm{H} 12 \mathrm{~B}$ & 114.9 & $\mathrm{H} 24 \mathrm{~B}-\mathrm{C} 24-\mathrm{H} 24 \mathrm{C}$ & 109.5 \\
\hline $\mathrm{C} 4-\mathrm{O} 2-\mathrm{C} 5$ & $104.94(19)$ & $\mathrm{C} 31-\mathrm{O} 6-\mathrm{H} 6$ & 109.5 \\
\hline $\mathrm{C} 3-\mathrm{O} 3-\mathrm{H} 3$ & 109.5 & $\mathrm{O} 6-\mathrm{C} 31-\mathrm{C} 32$ & $106.3(3)$ \\
\hline $\mathrm{C} 14-\mathrm{O} 4-\mathrm{H} 4$ & 109.5 & $\mathrm{O} 6-\mathrm{C} 31-\mathrm{C} 33$ & $109.7(3)$ \\
\hline $\mathrm{C} 9-\mathrm{N} 1-\mathrm{H} 1$ & $107.4(19)$ & $\mathrm{O} 6-\mathrm{C} 31-\mathrm{C} 34$ & $108.5(3)$ \\
\hline $\mathrm{C} 16-\mathrm{N} 1-\mathrm{H} 1$ & $104.0(18)$ & $\mathrm{C} 32-\mathrm{C} 31-\mathrm{C} 33$ & $111.3(3)$ \\
\hline $\mathrm{C} 16-\mathrm{N} 1-\mathrm{C} 9$ & $112.3(2)$ & $\mathrm{C} 32-\mathrm{C} 31-\mathrm{C} 34$ & $111.2(3)$ \\
\hline $\mathrm{C} 17-\mathrm{N} 1-\mathrm{H} 1$ & $106.9(18)$ & $\mathrm{C} 33-\mathrm{C} 31-\mathrm{C} 34$ & $109.8(4)$ \\
\hline $\mathrm{C} 17-\mathrm{N} 1-\mathrm{C} 9$ & $114.8(2)$ & $\mathrm{C} 31-\mathrm{C} 32-\mathrm{H} 32 \mathrm{~A}$ & 109.5 \\
\hline $\mathrm{C} 17-\mathrm{N} 1-\mathrm{C} 16$ & $110.7(2)$ & $\mathrm{C} 31-\mathrm{C} 32-\mathrm{H} 32 \mathrm{~B}$ & 109.5 \\
\hline $\mathrm{C} 2-\mathrm{C} 1-\mathrm{H} 1 \mathrm{~A}$ & 119.8 & $\mathrm{C} 31-\mathrm{C} 32-\mathrm{H} 32 \mathrm{C}$ & 109.5 \\
\hline $\mathrm{C} 2-\mathrm{C} 1-\mathrm{C} 11$ & $120.5(3)$ & $\mathrm{H} 32 \mathrm{~A}-\mathrm{C} 32-\mathrm{H} 32 \mathrm{~B}$ & 109.5 \\
\hline $\mathrm{C} 11-\mathrm{C} 1-\mathrm{H} 1 \mathrm{~A}$ & 119.8 & $\mathrm{H} 32 \mathrm{~A}-\mathrm{C} 32-\mathrm{H} 32 \mathrm{C}$ & 109.5 \\
\hline $\mathrm{C} 1-\mathrm{C} 2-\mathrm{H} 2$ & 118.9 & $\mathrm{H} 32 \mathrm{~B}-\mathrm{C} 32-\mathrm{H} 32 \mathrm{C}$ & 109.5 \\
\hline $\mathrm{C} 3-\mathrm{C} 2-\mathrm{C} 1$ & $122.2(3)$ & $\mathrm{C} 31-\mathrm{C} 33-\mathrm{H} 33 \mathrm{~A}$ & 109.5 \\
\hline $\mathrm{C} 3-\mathrm{C} 2-\mathrm{H} 2$ & 118.9 & $\mathrm{C} 31-\mathrm{C} 33-\mathrm{H} 33 \mathrm{~B}$ & 109.5 \\
\hline $\mathrm{O} 3-\mathrm{C} 3-\mathrm{C} 2$ & $125.6(3)$ & $\mathrm{C} 31-\mathrm{C} 33-\mathrm{H} 33 \mathrm{C}$ & 109.5 \\
\hline $\mathrm{O} 3-\mathrm{C} 3-\mathrm{C} 4$ & $118.0(2)$ & $\mathrm{H} 33 \mathrm{~A}-\mathrm{C} 33-\mathrm{H} 33 \mathrm{~B}$ & 109.5 \\
\hline $\mathrm{C} 4-\mathrm{C} 3-\mathrm{C} 2$ & $116.3(2)$ & $\mathrm{H} 33 \mathrm{~A}-\mathrm{C} 33-\mathrm{H} 33 \mathrm{C}$ & 109.5 \\
\hline $\mathrm{O} 2-\mathrm{C} 4-\mathrm{C} 3$ & $126.5(2)$ & $\mathrm{H} 33 \mathrm{~B}-\mathrm{C} 33-\mathrm{H} 33 \mathrm{C}$ & 109.5 \\
\hline $\mathrm{C} 12-\mathrm{C} 4-\mathrm{O} 2$ & $112.5(2)$ & $\mathrm{C} 31-\mathrm{C} 34-\mathrm{H} 34 \mathrm{~A}$ & 109.5 \\
\hline $\mathrm{C} 12-\mathrm{C} 4-\mathrm{C} 3$ & $121.0(3)$ & $\mathrm{C} 31-\mathrm{C} 34-\mathrm{H} 34 \mathrm{~B}$ & 109.5 \\
\hline $\mathrm{O} 2-\mathrm{C} 5-\mathrm{H} 5$ & 110.0 & $\mathrm{C} 31-\mathrm{C} 34-\mathrm{H} 34 \mathrm{C}$ & 109.5 \\
\hline $\mathrm{O} 2-\mathrm{C} 5-\mathrm{C} 6$ & $108.5(2)$ & $\mathrm{H} 34 \mathrm{~A}-\mathrm{C} 34-\mathrm{H} 34 \mathrm{~B}$ & 109.5 \\
\hline $\mathrm{O} 2-\mathrm{C} 5-\mathrm{C} 13$ & $104.7(2)$ & $\mathrm{H} 34 \mathrm{~A}-\mathrm{C} 34-\mathrm{H} 34 \mathrm{C}$ & 109.5 \\
\hline $\mathrm{C} 6-\mathrm{C} 5-\mathrm{H} 5$ & 110.0 & $\mathrm{H} 34 \mathrm{~B}-\mathrm{C} 34-\mathrm{H} 34 \mathrm{C}$ & 109.5 \\
\hline $\mathrm{O} 101-\mathrm{C} 106-\mathrm{C} 107-\mathrm{C} 108$ & $124.1(3)$ & $\mathrm{O} 1-\mathrm{C} 6-\mathrm{C} 7-\mathrm{C} 8$ & $129.6(3)$ \\
\hline $\mathrm{O} 102-\mathrm{C} 104-\mathrm{C} 112-\mathrm{C} 111$ & $175.1(3)$ & $\mathrm{O} 2-\mathrm{C} 4-\mathrm{C} 12-\mathrm{C} 11$ & $174.5(3)$ \\
\hline $\mathrm{O} 102-\mathrm{C} 104-\mathrm{C} 112-\mathrm{C} 113$ & $-6.0(3)$ & $\mathrm{O} 2-\mathrm{C} 4-\mathrm{C} 12-\mathrm{C} 13$ & $-7.9(3)$ \\
\hline $\mathrm{O} 102-\mathrm{C} 105-\mathrm{C} 106-\mathrm{O} 101$ & $-23.8(4)$ & $\mathrm{O} 2-\mathrm{C} 5-\mathrm{C} 6-\mathrm{O} 1$ & $-30.2(4)$ \\
\hline $\mathrm{O} 102-\mathrm{C} 105-\mathrm{C} 106-\mathrm{C} 107$ & $154.2(2)$ & $\mathrm{O} 2-\mathrm{C} 5-\mathrm{C} 6-\mathrm{C} 7$ & $149.9(2)$ \\
\hline $\mathrm{O} 102-\mathrm{C} 105-\mathrm{C} 113-\mathrm{C} 112$ & $-33.9(3)$ & $\mathrm{O} 2-\mathrm{C} 5-\mathrm{C} 13-\mathrm{C} 12$ & $-31.2(3)$ \\
\hline $\mathrm{O} 102-\mathrm{C} 105-\mathrm{C} 113-\mathrm{C} 114$ & $-151.0(2)$ & $\mathrm{O} 2-\mathrm{C} 5-\mathrm{C} 13-\mathrm{C} 14$ & $-148.7(2)$ \\
\hline $\mathrm{O} 102-\mathrm{C} 105-\mathrm{C} 113-\mathrm{C} 115$ & $80.3(3)$ & $\mathrm{O} 2-\mathrm{C} 5-\mathrm{C} 13-\mathrm{C} 15$ & $83.3(3)$ \\
\hline $\mathrm{O} 103-\mathrm{C} 103-\mathrm{C} 104-\mathrm{O} 102$ & $0.6(5)$ & $\mathrm{O} 3-\mathrm{C} 3-\mathrm{C} 4-\mathrm{O} 2$ & $0.8(4)$ \\
\hline $\mathrm{O} 103-\mathrm{C} 103-\mathrm{C} 104-\mathrm{C} 112$ & $179.8(3)$ & $\mathrm{O} 3-\mathrm{C} 3-\mathrm{C} 4-\mathrm{C} 12$ & $-179.4(3)$ \\
\hline $\mathrm{N} 101-\mathrm{C} 109-\mathrm{C} 110-\mathrm{C} 111$ & $-86.3(3)$ & $\mathrm{N} 1-\mathrm{C} 9-\mathrm{C} 10-\mathrm{C} 11$ & $-85.1(3)$ \\
\hline $\mathrm{N} 101-\mathrm{C} 109-\mathrm{C} 114-\mathrm{O} 104$ & $-48.8(3)$ & $\mathrm{N} 1-\mathrm{C} 9-\mathrm{C} 14-\mathrm{O} 4$ & $-49.5(3)$ \\
\hline $\mathrm{N} 101-\mathrm{C} 109-\mathrm{C} 114-\mathrm{C} 108$ & $-171.4(2)$ & $\mathrm{N} 1-\mathrm{C} 9-\mathrm{C} 14-\mathrm{C} 8$ & $-172.1(2)$ \\
\hline $\mathrm{N} 101-\mathrm{C} 109-\mathrm{C} 114-\mathrm{C} 113$ & $65.9(2)$ & $\mathrm{N} 1-\mathrm{C} 9-\mathrm{C} 14-\mathrm{C} 13$ & $66.1(3)$ \\
\hline $\mathrm{N} 101-\mathrm{C} 117-\mathrm{C} 118-\mathrm{C} 119$ & $-76.9(3)$ & $\mathrm{N} 1-\mathrm{C} 17-\mathrm{C} 18-\mathrm{C} 19$ & $-145.7(3)$ \\
\hline $\mathrm{N} 101-\mathrm{C} 117-\mathrm{C} 118-\mathrm{C} 120$ & $-146.1(3)$ & $\mathrm{N} 1-\mathrm{C} 17-\mathrm{C} 18-\mathrm{C} 20$ & $-76.9(4)$ \\
\hline $\mathrm{C} 101-\mathrm{C} 102-\mathrm{C} 103-\mathrm{O} 103$ & $-176.5(3)$ & $\mathrm{C} 1-\mathrm{C} 2-\mathrm{C} 3-\mathrm{O} 3$ & $-176.7(3)$ \\
\hline
\end{tabular}




\begin{tabular}{|c|c|c|c|}
\hline $\mathrm{C} 101-\mathrm{C} 102-\mathrm{C} 103-\mathrm{C} 104$ & $1.4(5)$ & $\mathrm{C} 1-\mathrm{C} 2-\mathrm{C} 3-\mathrm{C} 4$ & $1.0(4)$ \\
\hline $\mathrm{C} 101-\mathrm{C} 111-\mathrm{C} 112-\mathrm{C} 104$ & $3.0(5)$ & $\mathrm{C} 1-\mathrm{C} 11-\mathrm{C} 12-\mathrm{C} 4$ & $4.0(4)$ \\
\hline $\mathrm{C} 101-\mathrm{C} 111-\mathrm{C} 112-\mathrm{C} 113$ & $-175.6(3)$ & $\mathrm{C} 1-\mathrm{C} 11-\mathrm{C} 12-\mathrm{C} 13$ & $-173.1(3)$ \\
\hline $\mathrm{C} 102-\mathrm{C} 101-\mathrm{C} 111-\mathrm{C} 110$ & $176.2(3)$ & $\mathrm{C} 2-\mathrm{C} 1-\mathrm{C} 11-\mathrm{C} 10$ & $176.1(3)$ \\
\hline $\mathrm{C} 102-\mathrm{C} 101-\mathrm{C} 111-\mathrm{C} 112$ & $0.3(5)$ & $\mathrm{C} 2-\mathrm{C} 1-\mathrm{C} 11-\mathrm{C} 12$ & $-0.2(4)$ \\
\hline $\mathrm{C} 102-\mathrm{C} 103-\mathrm{C} 104-\mathrm{O} 102$ & $-177.4(3)$ & $\mathrm{C} 2-\mathrm{C} 3-\mathrm{C} 4-\mathrm{O} 2$ & $-177.2(3)$ \\
\hline $\mathrm{C} 102-\mathrm{C} 103-\mathrm{C} 104-\mathrm{C} 112$ & $1.8(4)$ & $\mathrm{C} 2-\mathrm{C} 3-\mathrm{C} 4-\mathrm{C} 12$ & $2.6(4)$ \\
\hline $\mathrm{C} 103-\mathrm{C} 104-\mathrm{C} 112-\mathrm{C} 111$ & $-4.2(5)$ & $\mathrm{C} 3-\mathrm{C} 4-\mathrm{C} 12-\mathrm{C} 11$ & $-5.4(5)$ \\
\hline $\mathrm{C} 103-\mathrm{C} 104-\mathrm{C} 112-\mathrm{C} 113$ & $174.6(3)$ & $\mathrm{C} 3-\mathrm{C} 4-\mathrm{C} 12-\mathrm{C} 13$ & $172.3(3)$ \\
\hline $\mathrm{C} 104-\mathrm{O} 102-\mathrm{C} 105-\mathrm{C} 106$ & $-88.5(3)$ & $\mathrm{C} 4-\mathrm{O} 2-\mathrm{C} 5-\mathrm{C} 6$ & $-93.1(2)$ \\
\hline $\mathrm{C} 104-\mathrm{O} 102-\mathrm{C} 105-\mathrm{C} 113$ & $32.1(3)$ & $\mathrm{C} 4-\mathrm{O} 2-\mathrm{C} 5-\mathrm{C} 13$ & $28.3(3)$ \\
\hline $\mathrm{C} 104-\mathrm{C} 112-\mathrm{C} 113-\mathrm{C} 105$ & $24.4(3)$ & $\mathrm{C} 4-\mathrm{C} 12-\mathrm{C} 13-\mathrm{C} 5$ & $24.1(3)$ \\
\hline $\mathrm{C} 104-\mathrm{C} 112-\mathrm{C} 113-\mathrm{C} 114$ & $147.5(2)$ & $\mathrm{C} 4-\mathrm{C} 12-\mathrm{C} 13-\mathrm{C} 14$ & $148.6(2)$ \\
\hline $\mathrm{C} 104-\mathrm{C} 112-\mathrm{C} 113-\mathrm{C} 115$ & $-92.5(3)$ & $\mathrm{C} 4-\mathrm{C} 12-\mathrm{C} 13-\mathrm{C} 15$ & $-92.5(3)$ \\
\hline $\mathrm{C} 105-\mathrm{O} 102-\mathrm{C} 104-\mathrm{C} 103$ & $162.4(3)$ & $\mathrm{C} 5-\mathrm{O} 2-\mathrm{C} 4-\mathrm{C} 3$ & $166.5(3)$ \\
\hline $\mathrm{C} 105-\mathrm{O} 102-\mathrm{C} 104-\mathrm{C} 112$ & $-16.9(3)$ & $\mathrm{C} 5-\mathrm{O} 2-\mathrm{C} 4-\mathrm{C} 12$ & $-13.3(3)$ \\
\hline $\mathrm{C} 105-\mathrm{C} 106-\mathrm{C} 107-\mathrm{C} 108$ & $-53.9(3)$ & $\mathrm{C} 5-\mathrm{C} 6-\mathrm{C} 7-\mathrm{C} 8$ & $-50.4(3)$ \\
\hline $\mathrm{C} 105-\mathrm{C} 113-\mathrm{C} 114-\mathrm{O} 104$ & $-78.5(3)$ & $\mathrm{C} 5-\mathrm{C} 13-\mathrm{C} 14-\mathrm{O} 4$ & $-77.4(3)$ \\
\hline $\mathrm{C} 105-\mathrm{C} 113-\mathrm{C} 114-\mathrm{C} 108$ & $43.1(3)$ & $\mathrm{C} 5-\mathrm{C} 13-\mathrm{C} 14-\mathrm{C} 8$ & $43.3(3)$ \\
\hline $\mathrm{C} 105-\mathrm{C} 113-\mathrm{C} 114-\mathrm{C} 109$ & $166.0(2)$ & $\mathrm{C} 5-\mathrm{C} 13-\mathrm{C} 14-\mathrm{C} 9$ & $166.2(2)$ \\
\hline $\mathrm{C} 105-\mathrm{C} 113-\mathrm{C} 115-\mathrm{C} 116$ & $-169.7(2)$ & $\mathrm{C} 5-\mathrm{C} 13-\mathrm{C} 15-\mathrm{C} 16$ & $-169.0(2)$ \\
\hline $\mathrm{C} 106-\mathrm{C} 105-\mathrm{C} 113-\mathrm{C} 112$ & $84.4(3)$ & $\mathrm{C} 6-\mathrm{C} 5-\mathrm{C} 13-\mathrm{C} 12$ & $86.9(3)$ \\
\hline $\mathrm{C} 106-\mathrm{C} 105-\mathrm{C} 113-\mathrm{C} 114$ & $-32.6(3)$ & $\mathrm{C} 6-\mathrm{C} 5-\mathrm{C} 13-\mathrm{C} 14$ & $-30.6(3)$ \\
\hline $\mathrm{C} 106-\mathrm{C} 105-\mathrm{C} 113-\mathrm{C} 115$ & $-161.3(2)$ & $\mathrm{C} 6-\mathrm{C} 5-\mathrm{C} 13-\mathrm{C} 15$ & $-158.6(2)$ \\
\hline $\mathrm{C} 106-\mathrm{C} 107-\mathrm{C} 108-\mathrm{C} 114$ & $62.1(3)$ & $\mathrm{C} 6-\mathrm{C} 7-\mathrm{C} 8-\mathrm{C} 14$ & $62.0(3)$ \\
\hline $\mathrm{C} 107-\mathrm{C} 108-\mathrm{C} 114-\mathrm{O} 104$ & $62.9(3)$ & $\mathrm{C} 7-\mathrm{C} 8-\mathrm{C} 14-\mathrm{O} 4$ & $61.7(3)$ \\
\hline $\mathrm{C} 107-\mathrm{C} 108-\mathrm{C} 114-\mathrm{C} 109$ & $-176.0(2)$ & $\mathrm{C} 7-\mathrm{C} 8-\mathrm{C} 14-\mathrm{C} 9$ & $-176.6(2)$ \\
\hline $\mathrm{C} 107-\mathrm{C} 108-\mathrm{C} 114-\mathrm{C} 113$ & $-56.6(3)$ & $\mathrm{C} 7-\mathrm{C} 8-\mathrm{C} 14-\mathrm{C} 13$ & $-57.4(3)$ \\
\hline $\mathrm{C} 109-\mathrm{N} 101-\mathrm{C} 116-\mathrm{C} 115$ & $57.1(3)$ & $\mathrm{C} 9-\mathrm{N} 1-\mathrm{C} 16-\mathrm{C} 15$ & $56.5(3)$ \\
\hline $\mathrm{C} 109-\mathrm{N} 101-\mathrm{C} 117-\mathrm{C} 118$ & $-60.2(3)$ & $\mathrm{C} 9-\mathrm{N} 1-\mathrm{C} 17-\mathrm{C} 18$ & $-59.8(3)$ \\
\hline $\mathrm{C} 109-\mathrm{C} 110-\mathrm{C} 111-\mathrm{C} 101$ & $176.2(3)$ & $\mathrm{C} 9-\mathrm{C} 10-\mathrm{C} 11-\mathrm{C} 1$ & $173.3(3)$ \\
\hline $\mathrm{C} 109-\mathrm{C} 110-\mathrm{C} 111-\mathrm{C} 112$ & $-8.0(4)$ & $\mathrm{C} 9-\mathrm{C} 10-\mathrm{C} 11-\mathrm{C} 12$ & $-10.4(4)$ \\
\hline $\mathrm{C} 110-\mathrm{C} 109-\mathrm{C} 114-\mathrm{O} 104$ & $-174.6(2)$ & $\mathrm{C} 10-\mathrm{C} 9-\mathrm{C} 14-\mathrm{O} 4$ & $-175.5(2)$ \\
\hline $\mathrm{C} 110-\mathrm{C} 109-\mathrm{C} 114-\mathrm{C} 108$ & $62.9(3)$ & $\mathrm{C} 10-\mathrm{C} 9-\mathrm{C} 14-\mathrm{C} 8$ & $61.9(3)$ \\
\hline $\mathrm{C} 110-\mathrm{C} 109-\mathrm{C} 114-\mathrm{C} 113$ & $-59.8(3)$ & $\mathrm{C} 10-\mathrm{C} 9-\mathrm{C} 14-\mathrm{C} 13$ & $-59.9(3)$ \\
\hline $\mathrm{C} 110-\mathrm{C} 111-\mathrm{C} 112-\mathrm{C} 104$ & $-173.4(3)$ & $\mathrm{C} 10-\mathrm{C} 11-\mathrm{C} 12-\mathrm{C} 4$ & $-172.6(3)$ \\
\hline $\mathrm{C} 110-\mathrm{C} 111-\mathrm{C} 112-\mathrm{C} 113$ & $8.0(5)$ & $\mathrm{C} 10-\mathrm{C} 11-\mathrm{C} 12-\mathrm{C} 13$ & $10.2(4)$ \\
\hline $\mathrm{C} 111-\mathrm{C} 101-\mathrm{C} 102-\mathrm{C} 103$ & $-2.4(5)$ & $\mathrm{C} 11-\mathrm{C} 1-\mathrm{C} 2-\mathrm{C} 3$ & $-2.2(5)$ \\
\hline $\mathrm{C} 111-\mathrm{C} 112-\mathrm{C} 113-\mathrm{C} 105$ & $-156.8(3)$ & $\mathrm{C} 11-\mathrm{C} 12-\mathrm{C} 13-\mathrm{C} 5$ & $-158.4(3)$ \\
\hline $\mathrm{C} 111-\mathrm{C} 112-\mathrm{C} 113-\mathrm{C} 114$ & $-33.7(4)$ & $\mathrm{C} 11-\mathrm{C} 12-\mathrm{C} 13-\mathrm{C} 14$ & $-33.9(4)$ \\
\hline $\mathrm{C} 111-\mathrm{C} 112-\mathrm{C} 113-\mathrm{C} 115$ & $86.3(3)$ & $\mathrm{C} 11-\mathrm{C} 12-\mathrm{C} 13-\mathrm{C} 15$ & $85.0(3)$ \\
\hline $\mathrm{C} 112-\mathrm{C} 113-\mathrm{C} 114-\mathrm{O} 104$ & $170.8(2)$ & $\mathrm{C} 12-\mathrm{C} 13-\mathrm{C} 14-\mathrm{O} 4$ & $170.5(2)$ \\
\hline $\mathrm{C} 112-\mathrm{C} 113-\mathrm{C} 114-\mathrm{C} 108$ & $-67.6(3)$ & $\mathrm{C} 12-\mathrm{C} 13-\mathrm{C} 14-\mathrm{C} 8$ & $-68.8(3)$ \\
\hline $\mathrm{C} 112-\mathrm{C} 113-\mathrm{C} 114-\mathrm{C} 109$ & $55.3(3)$ & $\mathrm{C} 12-\mathrm{C} 13-\mathrm{C} 14-\mathrm{C} 9$ & $54.1(3)$ \\
\hline $\mathrm{C} 112-\mathrm{C} 113-\mathrm{C} 115-\mathrm{C} 116$ & $-62.2(3)$ & $\mathrm{C} 12-\mathrm{C} 13-\mathrm{C} 15-\mathrm{C} 16$ & $-60.7(3)$ \\
\hline $\mathrm{C} 113-\mathrm{C} 105-\mathrm{C} 106-\mathrm{O} 101$ & $-139.6(3)$ & $\mathrm{C} 13-\mathrm{C} 5-\mathrm{C} 6-\mathrm{O} 1$ & $-146.1(3)$ \\
\hline $\mathrm{C} 113-\mathrm{C} 105-\mathrm{C} 106-\mathrm{C} 107$ & $38.5(3)$ & $\mathrm{C} 13-\mathrm{C} 5-\mathrm{C} 6-\mathrm{C} 7$ & $34.0(3)$ \\
\hline $\mathrm{C} 113-\mathrm{C} 115-\mathrm{C} 116-\mathrm{N} 101$ & $-52.1(3)$ & $\mathrm{C} 13-\mathrm{C} 15-\mathrm{C} 16-\mathrm{N} 1$ & $-52.5(3)$ \\
\hline
\end{tabular}




$\begin{array}{llll}\mathrm{C} 114-\mathrm{C} 109-\mathrm{C} 110-\mathrm{C} 111 & 35.6(4) & \mathrm{C} 14-\mathrm{C} 9-\mathrm{C} 10-\mathrm{C} 11 & 37.2(4) \\ \mathrm{C} 114-\mathrm{C} 113-\mathrm{C} 115-\mathrm{C} 116 & 57.6(3) & \mathrm{C} 14-\mathrm{C} 13-\mathrm{C} 15-\mathrm{C} 16 & 58.1(3) \\ \mathrm{C} 115-\mathrm{C} 113-\mathrm{C} 114-\mathrm{O} 104 & 51.4(3) & \mathrm{C} 15-\mathrm{C} 13-\mathrm{C} 14-\mathrm{O} 4 & 51.8(3) \\ \mathrm{C} 115-\mathrm{C} 113-\mathrm{C} 114-\mathrm{C} 108 & 173.0(2) & \mathrm{C} 15-\mathrm{C} 13-\mathrm{C} 14-\mathrm{C} 8 & 172.4(2) \\ \mathrm{C} 115-\mathrm{C} 113-\mathrm{C} 114-\mathrm{C} 109 & -64.1(3) & \mathrm{C} 15-\mathrm{C} 13-\mathrm{C} 14-\mathrm{C} 9 & -64.6(3) \\ \mathrm{C} 116-\mathrm{N} 101-\mathrm{C} 109-\mathrm{C} 110 & 62.6(3) & \mathrm{C} 16-\mathrm{N} 1-\mathrm{C} 9-\mathrm{C} 10 & 63.9(3) \\ \mathrm{C} 116-\mathrm{N} 101-\mathrm{C} 109-\mathrm{C} 114 & -64.2(3) & \mathrm{C} 16-\mathrm{N} 1-\mathrm{C} 9-\mathrm{C} 14 & -63.4(3) \\ \mathrm{C} 116-\mathrm{N} 101-\mathrm{C} 117-\mathrm{C} 118 & 170.5(2) & \mathrm{C} 16-\mathrm{N} 1-\mathrm{C} 17-\mathrm{C} 18 & 171.8(2) \\ \mathrm{C} 117-\mathrm{N} 101-\mathrm{C} 109-\mathrm{C} 110 & -66.3(3) & \mathrm{C} 17-\mathrm{N} 1-\mathrm{C} 9-\mathrm{C} 10 & -63.6(3) \\ \mathrm{C} 117-\mathrm{N} 101-\mathrm{C} 109-\mathrm{C} 114 & 166.9(2) & \mathrm{C} 17-\mathrm{N} 1-\mathrm{C} 9-\mathrm{C} 14 & 169.1(2) \\ \mathrm{C} 117-\mathrm{N} 101-\mathrm{C} 116-\mathrm{C} 115 & -172.2(2) & \mathrm{C} 17-\mathrm{N} 1-\mathrm{C} 16-\mathrm{C} 15 & -173.8(2) \\ \mathrm{C} 117-\mathrm{C} 118-\mathrm{C} 119-\mathrm{C} 120 & -106.7(3) & \mathrm{C} 17-\mathrm{C} 18-\mathrm{C} 19-\mathrm{C} 20 & 109.8(3) \\ \mathrm{C} 117-\mathrm{C} 118-\mathrm{C} 120-\mathrm{C} 119 & 110.0(3) & \mathrm{C} 17-\mathrm{C} 18-\mathrm{C} 20-\mathrm{C} 19 & -106.0(3)\end{array}$

Hydrogen-bond geometry $\left(\AA,{ }^{\circ}\right)$

\begin{tabular}{lllll}
\hline$D-\mathrm{H} \cdots A$ & $D-\mathrm{H}$ & $\mathrm{H} \cdots A$ & $D \cdots A$ & $D-\mathrm{H} \cdots A$ \\
\hline $\mathrm{N} 1-\mathrm{H} 1 \cdots \mathrm{C} 11^{\mathrm{i}}$ & $0.99(3)$ & $2.43(3)$ & $3.245(3)$ & $140(3)$ \\
$\mathrm{O} 3-\mathrm{H} 3 \cdots \mathrm{C} 11$ & $0.84(4)$ & $2.20(3)$ & $2.999(2)$ & $162(2)$ \\
$\mathrm{O} 4-\mathrm{H} 4 \cdots \mathrm{C} 2^{\mathrm{ii}}$ & $0.86(3)$ & $2.21(3)$ & $3.063(2)$ & $175(1)$ \\
$\mathrm{O} 5-\mathrm{H} 5 A \cdots \mathrm{O} 2$ & $0.87(5)$ & $2.09(3)$ & $2.902(3)$ & $154(4)$ \\
$\mathrm{O} 6-\mathrm{H} 6 \cdots \mathrm{O} 5$ & 0.84 & 2.03 & $2.867(4)$ & 174 \\
$\mathrm{~N} 101-\mathrm{H} 101 \cdots \mathrm{Cl} 2^{\mathrm{iii}}$ & $0.84(2)$ & $2.57(2)$ & $3.239(3)$ & $138(2)$ \\
$\mathrm{O} 103-\mathrm{H} 103 \cdots \mathrm{Cl} 2$ & $0.87(4)$ & $2.15(3)$ & $3.002(2)$ & $164(3)$ \\
$\mathrm{O} 104-\mathrm{H} 104 \cdots \mathrm{Cl} 1$ & $0.87(3)$ & $2.16(3)$ & $3.026(2)$ & $175(1)$ \\
\hline
\end{tabular}

Symmetry codes: (i) $-x+1, y+1 / 2,-z+1$; (ii) $x, y, z+1$; (iii) $-x, y-1 / 2,-z$. 